\title{
On the Stabilizing Effect of Convection in Three-Dimensional Incompressible Flows
}

\author{
THOMAS Y. HOU \\ California Institute of Technology \\ AND \\ ZHEN LEI \\ Fudan University
}

\begin{abstract}
We investigate the stabilizing effect of convection in three-dimensional incompressible Euler and Navier-Stokes equations. The convection term is the main source of nonlinearity for these equations. It is often considered destabilizing although it conserves energy due to the incompressibility condition. In this paper, we show that the convection term together with the incompressibility condition actually has a surprising stabilizing effect. We demonstrate this by constructing a new three-dimensional model that is derived for axisymmetric flows with swirl using a set of new variables. This model preserves almost all the properties of the full three-dimensional Euler or Navier-Stokes equations except for the convection term, which is neglected in our model. If we added the convection term back to our model, we would recover the full Navier-Stokes equations. We will present numerical evidence that seems to support that the three-dimensional model may develop a potential finite time singularity. We will also analyze the mechanism that leads to these singular events in the new three-dimensional model and how the convection term in the full Euler and Navier-Stokes equations destroys such a mechanism, thus preventing the singularity from forming in a finite time. (c) 2008 Wiley Periodicals, Inc.
\end{abstract}

\section{Introduction}

The question of whether a solution of the three-dimensional incompressible Navier-Stokes equations can develop a finite time singularity from smooth initial data with finite energy is one of the most outstanding open problems in mathematics [12]. A main difficulty in obtaining the global regularity of the threedimensional Navier-Stokes equations is due to the presence of the vortex-stretching term, which has a formal quadratic nonlinearity in vorticity. So far, most regularity analyses for the three-dimensional Navier-Stokes equations use energy estimates and require some kind of smallness assumption on the initial data [7, 23, 26, 33]. Due to the incompressibility condition, the convection term does not contribute to the energy norm of the velocity field or any $L^{p}(1<p \leq \infty)$ norm of the vorticity field. As a result, the convection term has been basically ignored in the regularity 
analysis for the Navier-Stokes equations. Most of the efforts have focused on how to use the diffusion term to control the nonlinear vortex-stretching term without making use of the convection term explicitly.

In this paper, we show that the convection term has a surprising stabilizing effect in the three-dimensional incompressible Euler and Navier-Stokes equations. It plays an essential role in depleting the vortex-stretching term. We demonstrate this stabilizing effect of convection by constructing a new three-dimensional model for axisymmetric flows with swirl. This model is formulated in terms of a set of new variables related to the angular velocity, the angular vorticity, and the angular stream function. The only difference between our three-dimensional model and the reformulated Navier-Stokes equations in terms of these new variables is that we neglect the convection term in the model. If we add the convection term back to the model, we will recover the full Navier-Stokes equations. This new threedimensional model preserves almost all the properties of the full three-dimensional Euler or Navier-Stokes equations. In particular, the strong solution of the model satisfies an energy identity similar to that of the full three-dimensional NavierStokes equations. We also prove a nonblowup criterion of Beale-Kato-Majda type [1] as well as a nonblowup criterion of Prodi-Serrin type [29, 31] for the model. In a subsequent paper, we will prove a new partial regularity result for the model [16], which is an analogue of the Caffarelli-Kohn-Nirenberg theory [2] for the full Navier-Stokes equations.

Despite the striking similarity at the theoretical level between our model and the Navier-Stokes equations, the former has a completely different behavior from the full Navier-Stokes equations. We will present numerical evidence which seems to support that the model may develop a potential finite time singularity from smooth initial data with finite energy. By exploiting the axisymmetric geometry of the problem, we obtain a very efficient adaptive solver with an optimal complexity that provides effective local resolutions of order $4096^{3}$ for the viscous model and $8192^{3}$ for the inviscid model. With this level of resolution, we obtain an excellent fit for the asymptotic blowup rate of maximum axial vorticity in the inviscid model. If we denote by $\omega^{z}$ the axial vorticity component along the $z$-direction, we find that $\left\|\omega^{z}\right\|_{\infty}(t) \approx C(T-t)^{-1}$ with a logarithmic correction, and the potential singularity approaches the symmetry axis (the $z$-axis) as $t \rightarrow T$. Moreover, our preliminary study seems to suggest that the potential singularity is locally selfsimilar and isotropic. We caution that the evidence for singularity formation of the inviscid model is not yet conclusive with the current level of resolutions. It requires higher numerical resolutions than what we have currently used to give more definitive evidence.

We also present numerical evidence which seems to suggest that the viscous model may develop a potential finite time singularity. The behavior of the nearly singular solution is similar to that of the solution of the inviscid model. We find that the solution of the viscous model experiences tremendous dynamic growth. The growth rate is much faster than what has been observed for the three-dimensional 
Navier-Stokes equations. On the other hand, we observe that the solution of the viscous model seems to be dominated by the dynamics of the inviscid model during the time interval of our computation. In order to determine whether the threedimensional model actually develops a finite time singularity and to study the local scaling property of the potential singularity, we need to solve the viscous model much closer to the potential singularity time to capture the viscous effect accurately. This would require substantially higher numerical resolutions than what we have used in the current paper. Depending on the local scaling property of the nearly singular solution and the balance between the vortex-stretching term and the viscous term, it is still possible that the viscous term may eventually regularize the nearly singular solution induced by the nonlinear vortex-stretching term. We will investigate this issue further in our future work.

To understand the mechanism for generating the potential finite time singularity, we monitor closely how the variables that contribute to the vortex-stretching term interact dynamically. Near the support of the maximum vorticity, which is defined as the region in which vorticity is comparable to its maximum, we find that the physical variables that contribute to the vortex-stretching term have a strong alignment locally. This seems to be the main mechanism for generating the potentially singular solution of the model.

To see how convection depletes the mechanism for generating a potential finite time singularity of our model, we add the convection term back to the model. We use the solution of the viscous model at a time sufficiently close to the potential singularity time as the initial condition for the full three-dimensional Navier-Stokes equations. Surprisingly, the solution of the three-dimensional Navier-Stokes equations immediately becomes defocused and smoother along the symmetry axis. As time increases, the solution develops a thin jet that moves away from the symmetry axis. As we know from the Caffarelli-Kohn-Nirenberg partial regularity theory [2] (see also [24] for a simplified proof), the three-dimensional axisymmetric NavierStokes equations cannot develop finite time singularities away from the symmetry axis. The fact that the convection term forces the most singular part of the solution to move away from the symmetry axis shows that convection has effectively destroyed the mechanism that leads to a potential finite time blowup observed in the model. Recent numerical study of the three-dimensional Euler equations by Hou and $\mathrm{Li}$ in $[14,15]$ shows that the convection term tends to introduce large deformation to the local vortex structure. The support of maximum vorticity becomes severely flattened as the vortex stretching intensifies in time. This anisotropic collapse of the support of maximum vorticity seems to play an essential role in depleting the vortex stretching. Some progress has been made recently along this direction; see [17].

The results presented in this paper may have some important implications for the global regularity of the three-dimensional Navier-Stokes equations. A successful strategy in analyzing the global regularity of the three-dimensional NavierStokes equations should take advantage of the stabilizing effect of the convection 
term in an essential way. So far most of the regularity analyses for the three-dimensional Navier-Stokes equations do not use the stabilizing effect of the convection term. In many cases, the same results can also be obtained for our model. We have presented numerical evidence in this paper which shows that the three-dimensional model is much more singular than the corresponding three-dimensional NavierStokes equations. New analytical tools that exploit the local geometric structure of the solution and the stabilizing effect of convection may be needed to prove the global regularity of the three-dimensional Navier-Stokes equations.

We also propose a generalized model in any dimension $d$ with $1 \leq d<\infty$. The three-dimensional model we discussed earlier corresponds to the special case of $d=5$ in the generalized model. Based on the balance between the vortexstretching term and the diffusion term, we can further classify the case of $d=4$ as the critical case, $1 \leq d<4$ as the subcritical case, and $d>4$ as the supercritical case. The generalized model in the supercritical case shares many difficulties found in the full Navier-Stokes equations. Global regularity of the generalized model in the supercritical case can be proved only for small initial data. On the other hand, we prove the global regularity of the generalized model in the critical and the subcritical cases.

We remark that this stabilizing effect of convection has been studied by Hou and $\mathrm{Li}$ in a recent paper [18]. They showed that convection plays an essential role in canceling the destabilizing vortex stretching in a new one-dimensional model that can be used to construct a family of exact solutions of the three-dimensional Euler or Navier-Stokes equations. This observation enabled them to obtain a crucial a priori pointwise estimate for a high-order norm of solutions in their model. Using this a priori estimate, they proved the global regularity of the three-dimensional Navier-Stokes equations for a family of large initial data whose solutions can lead to large dynamic growth yet have globally smooth solutions.

The stabilizing effect of convection has been also used in deriving localized nonblowup criteria for the three-dimensional incompressible Euler equations by Deng, Hou, and Yu in $[10,11]$. By using a Lagrangian formulation and exploiting the connection between vortex stretching and the local geometric regularity of vortex lines, they showed that the latter, even in an extremely localized region containing the maximum vorticity, can lead to depletion of vortex stretching, thus avoiding finite time singularities. Recently Okamoto and Ohkitani [27] investigated the role of the convection term in preventing the formation of singularities by studying several one-dimensional models and a two-dimensional model derived from the two-dimensional Euler equations. They also discussed other work where the idea has appeared in some form or other, including the work by Constantin [6] and by De Gregorio [8, 9], among others.

There has been some interesting development in the study of the three-dimensional incompressible Navier-Stokes equations and related models. In particular, by exploiting the special structure of the governing equations, Cao and Titi [3] 
proved the global well-posedness of the three-dimensional viscous primitive equations that model large-scale ocean and atmosphere dynamics. For the axisymmetric Navier-Stokes equations, Chen and others [4, 5] and Koch and others [21] recently proved that if $|u(x, t)| \leq C_{*}|t|^{-1 / 2}$ where $C_{*}$ is allowed to be large, then the velocity field $u$ is regular at time 0 .

The paper is organized as follows: In Section 2, we reformulate the threedimensional axisymmetric Navier-Stokes equations in terms of some new variables. In Section 3, we derive our three-dimensional model and prove the energy identity for this model. In Section 4, we generalize our model to an arbitrary space dimension and prove its global regularity in the subcritical and critical cases. Section 5 is devoted to the analysis of a special one-dimensional model. In Section 6, we present numerical evidence which seems to suggest that our three-dimensional model may develop a potential finite time singularity from some large smooth initial data with finite energy. We also analyze the mechanism for generating the potential finite time singularity for the three-dimensional model and demonstrate how the convection term destroys the mechanism that leads to the potential finite time blowup of the three-dimensional model. In Sections 7 and 8, we prove two nonblowup criteria for our model equations. The first one is an analogue of the well-known Beale-Kato-Majda nonblowup criterion, and the second one is an analogue of the Prodi-Serrin nonblowup criterion [29, 31].

\section{Reformulation of the Three-Dimensional Axisymmetric Navier-Stokes Equations}

Here we consider the three-dimensional axisymmetric incompressible NavierStokes equations with swirl

$$
\left\{\begin{array}{l}
\mathbf{u}_{t}+(\mathbf{u} \cdot \nabla) \mathbf{u}=-\nabla p+v \Delta \mathbf{u}, \\
\nabla \cdot \mathbf{u}=0, \\
\left.\mathbf{u}\right|_{t=0}=\mathbf{u}_{0}(x), \quad x=\left(x_{1}, x_{2}, z\right) .
\end{array}\right.
$$

Let

$$
e_{r}=\left(\frac{x_{1}}{r}, \frac{x_{2}}{r}, 0\right), \quad e_{\theta}=\left(-\frac{x_{2}}{r}, \frac{x_{1}}{r}, 0\right), \quad e_{z}=(0,0,1),
$$

be the three orthogonal unit vectors along the radial, the angular, and the axial directions, respectively, $r=\sqrt{x_{1}^{2}+x_{2}^{2}}$. We will decompose the velocity field as follows:

$$
\mathbf{u}=u^{r}(r, z, t) e_{r}+u^{\theta}(r, z, t) e_{\theta}+u^{z}(r, z, t) e_{z},
$$

where $u^{r}, u^{\theta}$, and $u^{z}$ designate the radial, angular, and axial velocity, respectively. In particular, $u^{\theta}$ is also referred to as the swirl component of the velocity. One can 
derive the following axisymmetric form of the Navier-Stokes equations in cylindrical coordinates [26]:

$$
\left\{\begin{array}{l}
\partial_{t} u^{\theta}+u^{r} \partial_{r} u^{\theta}+u^{z} \partial_{z} u^{\theta}=v\left(\Delta_{x}-\frac{1}{r^{2}}\right) u^{\theta}-\frac{u^{r} u^{\theta}}{r} \\
\partial_{t} \omega^{\theta}+u^{r} \partial_{r} \omega^{\theta}+u^{z} \partial_{z} \omega^{\theta}=v\left(\Delta_{x}-\frac{1}{r^{2}}\right) \omega^{\theta}+\partial_{z}\left(\frac{\left(u^{\theta}\right)^{2}}{r}\right)+\frac{u^{r} \omega^{\theta}}{r} \\
-\left(\Delta_{x}-\frac{1}{r^{2}}\right) \psi^{\theta}=\omega^{\theta}
\end{array}\right.
$$

where

$$
u^{r}=-\partial_{z} \psi^{\theta}, \quad u^{z}=\frac{1}{r} \partial_{r}\left(r \psi^{\theta}\right) .
$$

The incompressible constraint in cylindrical coordinates is given by

$$
\partial_{r} u^{r}+\partial_{z} u^{z}+\frac{u^{r}}{r}=0 \quad \text { or } \quad \partial_{r}\left(r u^{r}\right)+\partial_{z}\left(r u^{z}\right)=0,
$$

which is trivially satisfied in view of (2.4). In [25] Liu and Wang showed that if $\mathbf{u}$ is a smooth velocity field, then $u^{\theta}, \omega^{\theta}$, and $\psi^{\theta}$ must satisfy the following compatibility condition at $r=0$ :

$$
\left.u^{\theta}\right|_{r=0}=\left.\omega^{\theta}\right|_{r=0}=\left.\psi^{\theta}\right|_{r=0}=0
$$

The vorticity can be represented in cylindrical coordinates as follows:

$$
\boldsymbol{\omega}=-\left(u^{\theta}\right)_{z} e_{r}+\omega^{\theta} e_{\theta}+\frac{1}{r}\left(r u^{\theta}\right)_{r} e_{z} .
$$

Note that the axial vorticity component has the form

$$
\omega^{z}=\frac{1}{r}\left(r u^{\theta}\right)_{r}=\frac{u^{\theta}}{r}+\partial_{r} u^{\theta} .
$$

The last two terms on the right-hand side have the same asymptotic limit as $r \rightarrow 0$ since $\left.u^{\theta}\right|_{r=0}=0$. Thus the variable $\frac{u^{\theta}}{r}$ characterizes the axial vorticity near $r=0$.

In [18] Hou and $\mathrm{Li}$ introduced the variables

$$
u_{1}=\frac{u^{\theta}}{r}, \quad \omega_{1}=\frac{\omega^{\theta}}{r}, \quad \psi_{1}=\frac{\psi^{\theta}}{r},
$$

and derived the following equivalent system that governs the dynamics of $u_{1}, \omega_{1}$, and $\psi_{1}$ as follows:

$$
\left\{\begin{array}{l}
\partial_{t} u_{1}+u^{r} \partial_{r} u_{1}+u^{z} \partial_{z} u_{1}=v\left(\partial_{r}^{2}+\frac{3}{r} \partial_{r}+\partial_{z}^{2}\right) u_{1}+2 \partial_{z} \psi_{1} u_{1} \\
\partial_{t} \omega_{1}+u^{r} \partial_{r} \omega_{1}+u^{z} \partial_{z} \omega_{1}=v\left(\partial_{r}^{2}+\frac{3}{r} \partial_{r}+\partial_{z}^{2}\right) \omega_{1}+\partial_{z}\left(\left(u_{1}\right)^{2}\right) \\
-\left(\partial_{r}^{2}+\frac{3}{r} \partial_{r}+\partial_{z}^{2}\right) \psi_{1}=\omega_{1}
\end{array}\right.
$$

where

$$
u^{r}=-\partial_{z}\left(r \psi_{1}\right), \quad u^{z}=\frac{1}{r} \partial_{r}\left(r^{2} \psi_{1}\right)
$$


Note that in the new system (2.9)-(2.10), the convection term has absorbed one of the vortex-stretching terms, $\frac{u^{r} \omega^{\theta}}{r}$, which originally appears in the second equation of (2.3). In some sense, the convection term has already stabilized one of the potentially destabilized vortex-stretching terms in the above reformulation.

Finally, we recall the basic energy identity for the Navier-Stokes equations (2.1):

$$
\frac{1}{2} \frac{d}{d t} \int_{\mathbb{R}^{3}}|\mathbf{u}|^{2} d x+v \int_{\mathbb{R}^{3}}|\nabla \mathbf{u}|^{2} d x=0 .
$$

Naturally, the system (2.3)-(2.4) and the new system (2.9)-(2.10) enjoy the same energy identity (2.11).

\section{A New Three-Dimensional Model and Its Properties}

In this section, we introduce our three-dimensional model for axisymmetric flows with swirl. The purpose of introducing this model is to study the stabilizing effect of the convection term in the three-dimensional incompressible Euler or Navier-Stokes equations. Our model shares many properties with the threedimensional Euler or Navier-Stokes equations. First of all, it has the same nonlinear vortex-stretching term. Second, it has the same type of a priori energy identity. Third, almost all the existing nonblowup criteria for the three-dimensional Euler or Navier-Stokes equations are also valid for our model.

A three-dimensional model that satisfies all these properties seems hard to find in general. But in terms of the equations for the new variables, $u_{1}, \omega_{1}$, and $\psi_{1}$, we can get our three-dimensional model equations by simply dropping the convection term from (2.9):

$$
\left\{\begin{array}{l}
\partial_{t} u_{1}=v\left(\partial_{r}^{2}+\frac{3}{r} \partial_{r}+\partial_{z}^{2}\right) u_{1}+2 \partial_{z} \psi_{1} u_{1} \\
\partial_{t} \omega_{1}=v\left(\partial_{r}^{2}+\frac{3}{r} \partial_{r}+\partial_{z}^{2}\right) \omega_{1}+\partial_{z}\left(u_{1}^{2}\right) \\
-\left(\partial_{r}^{2}+\frac{3}{r} \partial_{r}+\partial_{z}^{2}\right) \psi_{1}=\omega_{1}
\end{array}\right.
$$

Note that (3.1) is already a closed system. The main difference between our three-dimensional model and the original Navier-Stokes equations is that we neglect the convection term in our model. If we add the convection term back to our three-dimensional model, we will recover the Navier-Stokes equations.

Below we will derive some important properties of the model equations (3.1).

First of all, we note that there is an intrinsic incompressible structure in the three-dimensional model equations (3.1). To see this, we define the velocity field as

$$
\mathbf{u}=u^{r} e_{r}+u^{\theta} e_{\theta}+u^{z} e_{z}
$$

with

$$
u^{r}=-\left(r \psi_{1}\right)_{z}, \quad u^{\theta}=r u_{1}, \quad u^{z}=\frac{\left(r^{2} \psi_{1}\right)_{r}}{r}
$$


It is easy to check that

$$
\nabla \cdot \mathbf{u}=\partial_{r} u^{r}+\partial_{z} u^{z}+\frac{u^{r}}{r}=0
$$

which is the same incompressibility condition for the original incompressible Euler or Navier-Stokes equations.

Next, we will prove a compatibility condition for the solution of our threedimensional model. This compatibility condition was first obtained by Liu and Wang in [25] for the three-dimensional axisymmetric Euler or Navier-Stokes equations.

PROPOSITION 3.1 Any smooth solution $\left(u_{1}, \omega_{1}, \psi_{1}\right)$ to the three-dimensional viscous model equations (3.1) on $0 \leq t<T$ with $v>0$ satisfies the compatibility conditions

$$
\left.\partial_{r}^{k} u^{\theta}(t, r, z)\right|_{r=0}=\left.\partial_{r}^{k} \omega^{\theta}(t, r, z)\right|_{r=0}=\left.\partial_{r}^{k} \psi^{\theta}(t, r, z)\right|_{r=0}=0
$$

for all even integers $k \geq 0$ and $0 \leq t<T$ where

$$
u^{\theta}=r u_{1}, \quad \omega^{\theta}=r \omega_{1}, \quad \psi^{\theta}=r \psi_{1} .
$$

PROOF: Let $\left(u_{1}, \omega_{1}, \psi_{1}\right)$ be a smooth solution of (3.1) for $0 \leq t<T$. It is easy to see that $\left(u^{\theta}, \omega^{\theta}, \psi^{\theta}\right)$ satisfies

$$
\left\{\begin{array}{l}
\partial_{t} u^{\theta}=v\left(\partial_{r}^{2}+\frac{1}{r} \partial_{r}+\partial_{z}^{2}-\frac{1}{r^{2}}\right) u^{\theta}+\frac{2 \partial_{z} \psi^{\theta}}{r} u^{\theta} \\
\partial_{t} \omega^{\theta}=v\left(\partial_{r}^{2}+\frac{1}{r} \partial_{r}+\partial_{z}^{2}-\frac{1}{r^{2}}\right) \omega^{\theta}+\partial_{z} \frac{\left(u^{\theta}\right)^{2}}{r} \\
-\left(\partial_{r}^{2}+\frac{1}{r} \partial_{r}+\partial_{z}^{2}-\frac{1}{r^{2}}\right) \psi^{\theta}=\omega^{\theta}
\end{array}\right.
$$

for $r>0$. Multiplying each equation in (3.7) by $r^{2}$ yields

$$
\left\{\begin{array}{l}
r^{2} \partial_{t} u^{\theta}=v\left(r^{2} \partial_{r}^{2}+r \partial_{r}+r^{2} \partial_{z}^{2}-1\right) u^{\theta}+2 r \partial_{z} \psi^{\theta} u^{\theta} \\
r^{2} \partial_{t} \omega^{\theta}=v\left(r^{2} \partial_{r}^{2}+r \partial_{r}+r^{2} \partial_{z}^{2}-1\right) \omega^{\theta}+r \partial_{z}\left[\left(u^{\theta}\right)^{2}\right], \\
-\left(r^{2} \partial_{r}^{2}+r \partial_{r}+r^{2} \partial_{z}^{2}-1\right) \psi^{\theta}=r^{2} \omega^{\theta} .
\end{array}\right.
$$

Since $\left(u_{1}, \omega_{1}, \psi_{1}\right)$ is smooth, so is $\left(u^{\theta}, \omega^{\theta}, \psi^{\theta}\right)$. By letting $r \rightarrow 0$ in (3.7), we prove the compatibility condition (3.5) for $k=0$. Next we differentiate (3.7) with respect to $r$ twice, then let $r \rightarrow 0$. We can see that (3.5) is true for $k=2$. By using an induction argument, one can prove that (3.5) is true for all even $k>2$.

Remark 3.2. We remark that the above compatibility condition is necessary if we require that the reconstructed three-dimensional velocity field using (3.2)-(3.3) be smooth at $r=0$. This is due to the fact that the unit vectors $e_{r}$ and $e_{\theta}$ are singular at $r=0$, but $r^{2 k+1} e_{r}$ and $r^{2 k+1} e_{\theta}$ are regular at $r=0$. Thus $u^{\theta}, \omega^{\theta}$, and $\psi^{\theta}$ must have asymptotic expansions in terms of the odd powers of $r$ near $r=0$. This would give rise to the compatibility condition (3.5), even in the case of $v=0$. 
Notation. To make it easier to compare the three-dimensional model equations (3.1) with the axisymmetric Navier-Stokes equations (2.9)-(2.10), we recast our three-dimensional model equations in $\mathbb{R} \times \mathbb{R}^{5}$ and introduce some notation. We will denote by $x=\left(x_{1}, x_{2}, z\right)$ a point in $\mathbb{R}^{3}$ and $y=\left(y_{1}, y_{2}, y_{3}, y_{4}, z\right)$ a point in $\mathbb{R}^{5}$. The time derivative is denoted by $\partial_{t}$. The space derivative with respect to $x$ or $y$ is denoted by $\nabla_{x}=\left(\partial_{x_{1}}, \partial_{x_{2}}, \partial_{z}\right)^{\top}$ in $\mathbb{R}^{3}$ or by $\nabla_{y}=\left(\partial_{y_{1}}, \partial_{y_{2}}, \partial_{y_{3}}, \partial_{y_{4}}, \partial_{z}\right)^{\top}$ in $\mathbb{R}^{5}$, respectively. Similarly, we will use

$$
\Delta_{x}=\partial_{x_{1}}^{2}+\partial_{x_{2}}^{2}+\partial_{z}^{2}, \quad \Delta_{y}=\partial_{y_{1}}^{2}+\partial_{y_{2}}^{2}+\partial_{y_{3}}^{2}+\partial_{y_{4}}^{2}+\partial_{z}^{2}
$$

Throughout this paper, if the function is axisymmetric, we will denote its space variable by $(r, z)$, where the $z$-axis is the symmetry axis and $r=\sqrt{x_{1}^{2}+x_{2}^{2}}$ in $\mathbb{R}^{3}$ and $r=\sqrt{y_{1}^{2}+y_{2}^{2}+y_{3}^{2}+y_{4}^{2}}$ in $\mathbb{R}^{5}$. In particular, the Laplacians using cylindrical coordinates are

$$
\Delta_{x}=\partial_{r}^{2}+\frac{1}{r} \partial_{r}+\partial_{z}^{2}, \quad \Delta_{y}=\partial_{r}^{2}+\frac{3}{r} \partial_{r}+\partial_{z}^{2}
$$

Now we state the important energy identity of the three-dimensional model equations (3.1), which is equivalent to (2.11) of the axisymmetric Navier-Stokes equations.

PROPOSITION 3.3 The solution of the three-dimensional model equations (3.1) satisfies the energy identity

$$
\begin{aligned}
& \frac{1}{2} \frac{d}{d t} \int_{-\infty}^{\infty} \int_{0}^{\infty}\left(\left|u_{1}\right|^{2}+2\left|\nabla_{y} \psi_{1}\right|^{2}\right) r^{3} d r d z \\
& \quad+v \int_{-\infty}^{\infty} \int_{0}^{\infty}\left(\left|\nabla_{y} u_{1}\right|^{2}+2\left|\Delta_{y} \psi_{1}\right|^{2}\right) r^{3} d r d z=0
\end{aligned}
$$

Moreover, we have

$$
\begin{aligned}
& \int_{-\infty}^{\infty} \int_{0}^{\infty}\left(\left|u_{1}\right|^{2}+2\left|\nabla_{y} \psi_{1}\right|^{2}\right) r^{3} d r d z \\
& \quad=\int_{-\infty}^{\infty} \int_{0}^{\infty}\left(2|\mathbf{u}|^{2}-\left|u^{\theta}\right|^{2}\right) r d r d z \\
& \int_{-\infty}^{\infty} \int_{0}^{\infty}\left(\left|\nabla_{y} u_{1}\right|^{2}+2\left|\Delta_{y} \psi_{1}\right|^{2}\right) r^{3} d r d z \\
& \quad=\int_{-\infty}^{\infty} \int_{0}^{\infty}\left(2\left|\nabla_{x} \mathbf{u}\right|^{2}-\left(\left|\partial_{r} u^{\theta}\right|^{2}+\left|\partial_{z} u^{\theta}\right|^{2}+\left|\frac{u^{\theta}}{r}\right|^{2}\right)\right) r d r d z
\end{aligned}
$$


where $\mathbf{u}$ is defined in (3.2)-(3.3). Furthermore, the energy identity (3.9) is equivalent to that of the Navier-Stokes equations in the sense that

$$
\begin{aligned}
\int_{-\infty}^{\infty} \int_{0}^{\infty}|\mathbf{u}|^{2} r d r d z & \leq \int_{-\infty}^{\infty} \int_{0}^{\infty}\left(\left|u_{1}\right|^{2}+2\left|\nabla_{y} \psi_{1}\right|^{2}\right) r^{3} d r d z \\
& \leq 2 \int_{-\infty}^{\infty} \int_{0}^{\infty}|\mathbf{u}|^{2} r d r d z \\
\int_{-\infty}^{\infty} \int_{0}^{\infty}\left|\nabla_{x} \mathbf{u}\right|^{2} r d r d z & \leq \int_{-\infty}^{\infty} \int_{0}^{\infty}\left(\left|\nabla_{y} u_{1}\right|^{2}+2\left|\Delta_{y} \psi_{1}\right|^{2}\right) r^{3} d r d z \\
& \leq 2 \int_{-\infty}^{\infty} \int_{0}^{\infty}\left|\nabla_{x} \mathbf{u}\right|^{2} r d r d z
\end{aligned}
$$

Proof: The energy identity (3.9) follows by the standard energy method. Observe that the diffusion operator in the three-dimensional model equations, which is given by $\partial_{r}^{2}+\frac{3}{r} \partial_{r}+\partial_{z}^{2}$, is actually the Laplacian operator in $\mathbb{R}^{5}, \Delta_{y}$. Thus, it would be easier to prove the energy identity by performing energy estimates in $\mathbb{R}^{5}$.

Multiplying the first equation of (3.1) by $u_{1}$ and then integrating over $\mathbb{R}^{5}$ yields

$$
\frac{1}{2} \frac{d}{d t} \int_{\mathbb{R}^{5}} u_{1}^{2} d y+v \int_{\mathbb{R}^{5}}\left|\nabla_{y} u_{1}\right|^{2} d y=\int_{\mathbb{R}^{5}} 2 \partial_{z} \psi_{1} u_{1}^{2} d y .
$$

Multiplying the second equation in (3.1) by $\psi_{1}$ and then integrating over $\mathbb{R}^{5}$ yields

$$
\int_{\mathbb{R}^{5}} \partial_{t} \omega_{1} \psi_{1} d y-v \int_{\mathbb{R}^{5}} \psi_{1} \Delta_{y} \omega_{1} d y=\int_{\mathbb{R}^{5}} \psi_{1} \partial_{z}\left(u_{1}^{2}\right) d y .
$$

Using the third equation in (3.1) and integration by parts, we have

$$
\frac{1}{2} \frac{d}{d t} \int_{\mathbb{R}^{5}}\left|\nabla_{y} \psi_{1}\right|^{2} d y+v \int_{\mathbb{R}^{5}}\left|\Delta_{y} \psi_{1}\right|^{2} d y=-\int_{\mathbb{R}^{5}} \partial_{z} \psi_{1} u_{1}^{2} d y .
$$

Multiplying (3.15) by 2 and adding the resulting equation to (3.14) gives (3.9).

Next, we prove (3.10)-(3.11). The equality (3.10) follows by the following straightforward calculation:

$$
\begin{aligned}
& \int_{-\infty}^{\infty} \int_{0}^{\infty}\left(\left|u_{1}\right|^{2}+2\left|\nabla_{y} \psi_{1}\right|^{2}\right) r^{3} d r d z \\
& \quad=\int_{-\infty}^{\infty} \int_{0}^{\infty}\left(\left|u_{1}\right|^{2}+2\left|\partial_{r} \psi_{1}\right|^{2}+2\left|\partial_{z} \psi_{1}\right|^{2}\right) r^{3} d r d z \\
& \quad=\int_{-\infty}^{\infty} \int_{0}^{\infty}\left(\left|u^{\theta}\right|^{2}+2\left|\partial_{r} \psi^{\theta}-\frac{\psi^{\theta}}{r}\right|^{2}+2\left|u^{r}\right|^{2}\right) r d r d z=
\end{aligned}
$$




$$
\begin{aligned}
& =\int_{-\infty}^{\infty} \int_{0}^{\infty}\left(\left|u^{\theta}\right|^{2}+2\left|\partial_{r} \psi^{\theta}+\frac{\psi^{\theta}}{r}\right|^{2}+2\left|u^{r}\right|^{2}\right) r d r d z \\
& =\int_{-\infty}^{\infty} \int_{0}^{\infty}\left(\left|u^{\theta}\right|^{2}+2\left|u^{r}\right|^{2}+2\left|u^{z}\right|^{2}\right) r d r d z
\end{aligned}
$$

where we have used Proposition 3.1 and the identity

$$
\int_{-\infty}^{\infty} \int_{0}^{\infty} \psi^{\theta} \partial_{r} \psi^{\theta} d r d z=0
$$

Using (3.10), we can easily obtain (3.12).

It remains to prove (3.11) and (3.13). First, we compute $\nabla_{x} \mathbf{u}$ as follows:

$$
\begin{aligned}
\nabla_{x} \mathbf{u}= & \left(e_{r} \partial_{r}+\frac{1}{r} e_{\theta} \partial_{\theta}+e_{z} \partial_{z}\right)\left(u^{r} e_{r}+u^{\theta} e_{\theta}+u^{z} e_{z}\right) \\
= & \partial_{r} u^{r} e_{r} \otimes e_{r}+\partial_{r} u^{\theta} e_{r} \otimes e_{\theta}+\partial_{r} u^{z} e_{r} \otimes e_{z} \\
& -\frac{u^{\theta}}{r} e_{\theta} \otimes e_{r}+\frac{u^{r}}{r} e_{\theta} \otimes e_{\theta} \\
& +\partial_{z} u^{r} e_{z} \otimes e_{r}+\partial_{z} u^{\theta} e_{z} \otimes e_{\theta}+\partial_{z} u^{z} e_{z} \otimes e_{z} .
\end{aligned}
$$

Thus, by the definitions of $u^{r}$ and $u^{z}$ in (3.3) and the incompressibility constraint (3.4), we have

$$
\begin{aligned}
\left|\nabla_{x} \mathbf{u}\right|^{2}= & \left|\partial_{r} u^{\theta}\right|^{2}+\left|\partial_{z} u^{\theta}\right|^{2}+\left|\frac{u^{\theta}}{r}\right|^{2} \\
& +\left|\partial_{r} u^{r}\right|^{2}+\left|\partial_{z} u^{r}\right|^{2}+\left|\frac{u^{r}}{r}\right|^{2}+\left|\partial_{r} u^{z}\right|^{2}+\left|\partial_{z} u^{z}\right|^{2} \\
= & \left|\partial_{r} u^{\theta}\right|^{2}+\left|\partial_{z} u^{\theta}\right|^{2}+\left|\frac{u^{\theta}}{r}\right|^{2}+2\left|\frac{u^{r}}{r}\right|^{2} \\
& -2 \partial_{r} u^{r} \partial_{z} u^{z}+\left|\partial_{r} u^{z}\right|^{2}+\left|\partial_{z} u^{r}\right|^{2} \\
= & \left(\left|\partial_{r} u^{\theta}\right|^{2}+\left|\partial_{z} u^{\theta}\right|^{2}+\left|\frac{u^{\theta}}{r}\right|^{2}\right)+\left|\partial_{r}^{2} \psi^{\theta}\right|^{2}+\left|\partial_{z}^{2} \psi^{\theta}\right|^{2} \\
& +2\left|\partial_{r z}^{2} \psi^{\theta}\right|^{2}+\left[\partial_{r}\left(\frac{\psi^{\theta}}{r}\right)\right]^{2}+2\left[\partial_{z}\left(\frac{\psi^{\theta}}{r}\right)\right]^{2} \\
& +2 \partial_{r}^{2} \psi^{\theta} \partial_{r}\left(\frac{\psi^{\theta}}{r}\right)+2 \partial_{r z}^{2} \psi^{\theta} \partial_{z}\left(\frac{\psi^{\theta}}{r}\right) .
\end{aligned}
$$


On the other hand, Proposition 3.1 and a simple computation give

$$
\begin{aligned}
\int_{-\infty}^{\infty} \int_{0}^{\infty}\left|\nabla_{y} u_{1}\right|^{2} r^{3} d r d z= & \\
& \int_{-\infty}^{\infty} \int_{0}^{\infty}\left(\left|\partial_{r} u^{\theta}\right|^{2}+\left|\partial_{z} u^{\theta}\right|^{2}+\left|\frac{u^{\theta}}{r}\right|^{2}\right) r d r d z
\end{aligned}
$$

Note that

$$
\begin{aligned}
\left|\Delta_{y} \psi_{1}\right|^{2}= & \sum_{i, j=1}^{4}\left|\partial_{i j}^{2} \psi_{1}\right|^{2}+2\left|\partial_{r z}^{2} \psi_{1}\right|^{2}+\left|\partial_{z}^{2} \psi_{1}\right|^{2} \\
= & \sum_{i, j=1}^{4}\left|\partial_{i j}^{2} \psi_{1}\right|^{2} \\
& +\frac{1}{r^{2}}\left(\left|\partial_{z}^{2} \psi^{\theta}\right|^{2}+2\left|\partial_{r z}^{2} \psi^{\theta}\right|^{2}\right. \\
& \left.+2\left[\partial_{z}\left(\frac{\psi^{\theta}}{r}\right)\right]^{2}-4 \partial_{r z}^{2} \psi^{\theta} \partial_{z}\left(\frac{\psi^{\theta}}{r}\right)\right) .
\end{aligned}
$$

A straightforward calculation gives

$$
\begin{aligned}
& \sum_{i, j=1}^{4}\left|\partial_{i j}^{2} \psi_{1}\right|^{2}= \sum_{i, j=1}^{4}\left|\partial_{i}\left(\frac{y_{j}}{r} \partial_{r} \psi_{1}\right)\right|^{2} \\
&= \sum_{i, j=1}^{4}\left|\frac{\delta_{i j}}{r} \partial_{r} \psi_{1}-\frac{y_{i} y_{j}}{r^{3}} \partial_{r} \psi_{1}+\frac{y_{i} y_{j}}{r^{2}} \partial_{r}^{2} \psi_{1}\right|^{2} \\
&= \sum_{i, j=1}^{4}\left|\frac{\delta_{i j}}{r} \partial_{r} \psi_{1}-\frac{3 y_{i} y_{j}}{r^{3}} \partial_{r} \psi_{1}+\frac{y_{i} y_{j}}{r^{3}} \partial_{r}^{2} \psi^{\theta}\right|^{2} \\
&= \frac{1}{r^{2}}\left|\partial_{r}^{2} \psi^{\theta}\right|^{2}+\frac{13}{r^{2}}\left(\partial_{r} \psi_{1}\right)^{2} \\
&+2 \sum_{i, j=1}^{4}\left(-\frac{3 y_{i} y_{j} \delta_{i j}}{r^{2}} \frac{1}{r^{2}}\left(\partial_{r} \psi_{1}\right)^{2}+\frac{y_{i} y_{j}}{r^{2}} \frac{\delta_{i j}}{r^{2}} \partial_{r} \psi_{1} \partial_{r}^{2} \psi^{\theta}\right. \\
&\left.\quad-\frac{3 y_{i} y_{j}}{r^{2}} \frac{y_{i} y_{j}}{r^{2}} \frac{1}{r^{2}} \partial_{r} \psi_{1} \partial_{r}^{2} \psi^{\theta}\right) \\
&=\frac{1}{r^{2}}\left(\left|\partial_{r}^{2} \psi^{\theta}\right|^{2}+7\left|\partial_{r} \psi_{1}\right|^{2}-4 \partial_{r} \psi_{1} \partial_{r}^{2} \psi^{\theta}\right) .
\end{aligned}
$$


Therefore, we obtain

$$
\begin{aligned}
\left|\Delta_{y} \psi_{1}\right|^{2}=\frac{1}{r^{2}}( & \left|\partial_{r}^{2} \psi^{\theta}\right|^{2}+\left|\partial_{z}^{2} \psi^{\theta}\right|^{2}+2\left|\partial_{r z}^{2} \psi^{\theta}\right|^{2} \\
& +2\left[\partial_{z}\left(\frac{\psi^{\theta}}{r}\right)\right]^{2}+7\left[\partial_{r}\left(\frac{\psi^{\theta}}{r}\right)\right]^{2} \\
& \left.-4 \partial_{z}\left(\frac{\psi^{\theta}}{r}\right) \partial_{r z}^{2} \psi^{\theta}-4 \partial_{r}\left(\frac{\psi^{\theta}}{r}\right) \partial_{r}^{2} \psi^{\theta}\right) .
\end{aligned}
$$

Combining (3.18) and (3.19), we have

$$
\begin{gathered}
\int_{-\infty}^{\infty} \int_{0}^{\infty}\left(\left|\nabla_{y} u_{1}\right|^{2}+2\left|\Delta_{y} \psi_{1}\right|^{2}\right) r^{3} d r d z \\
=\int_{-\infty}^{\infty} \int_{0}^{\infty}\left[\left|\partial_{r} u^{\theta}\right|^{2}+\left|\partial_{z} u^{\theta}\right|^{2}+\left|\frac{u^{\theta}}{r}\right|^{2}\right. \\
+2\left(\left|\partial_{r}^{2} \psi^{\theta}\right|^{2}+\left|\partial_{z}^{2} \psi^{\theta}\right|^{2}+2\left|\partial_{r z}^{2} \psi^{\theta}\right|^{2}\right. \\
+2\left[\partial_{z}\left(\frac{\psi^{\theta}}{r}\right)\right]^{2}+7\left[\partial_{r}\left(\frac{\psi^{\theta}}{r}\right)\right]^{2} \\
\left.\left.-4 \partial_{z}\left(\frac{\psi^{\theta}}{r}\right) \partial_{r z}^{2} \psi^{\theta}-4 \partial_{r}\left(\frac{\psi^{\theta}}{r}\right) \partial_{r}^{2} \psi^{\theta}\right)\right] r d r d z
\end{gathered}
$$

By comparing (3.17) with (3.20), we have

$$
\begin{aligned}
& \int_{-\infty}^{\infty} \int_{0}^{\infty}\left(\left|\nabla_{y} u_{1}\right|^{2}+2\left|\Delta_{y} \psi_{1}\right|^{2}\right) r^{3} d r d z \\
& =\int_{-\infty}^{\infty} \int_{0}^{\infty}\left(2\left|\nabla_{x} \mathbf{u}\right|^{2}-\left(\left|\partial_{r} u^{\theta}\right|^{2}+\left|\partial_{r} u^{\theta}\right|^{2}+\left|\frac{u^{\theta}}{r}\right|^{2}\right)\right) r d r d z \\
& +12 \int_{-\infty}^{\infty} \int_{0}^{\infty}\left(\left[\partial_{r}\left(\frac{\psi^{\theta}}{r}\right)\right]^{2}\right. \\
& \left.\quad-\partial_{z}\left(\frac{\psi^{\theta}}{r}\right) \partial_{r z}^{2} \psi^{\theta}-\partial_{r}\left(\frac{\psi^{\theta}}{r}\right) \partial_{r}^{2} \psi^{\theta}\right) r d r d z
\end{aligned}
$$

Thus to prove (3.11), it suffices to show that

$$
\begin{aligned}
\int_{-\infty}^{\infty} \int_{0}^{\infty}( & {\left[\partial_{r}\left(\frac{\psi^{\theta}}{r}\right)\right]^{2} } \\
& \left.-\partial_{z}\left(\frac{\psi^{\theta}}{r}\right) \partial_{r z}^{2} \psi^{\theta}-\partial_{r}\left(\frac{\psi^{\theta}}{r}\right) \partial_{r}^{2} \psi^{\theta}\right) r d r d z=0
\end{aligned}
$$


Indeed, direct calculations give

$$
\begin{aligned}
\int_{-\infty}^{\infty} \int_{0}^{\infty}\left(\left[\partial_{r}\left(\frac{\psi^{\theta}}{r}\right)\right]^{2}-\partial_{z}\left(\frac{\psi^{\theta}}{r}\right) \partial_{r z}^{2} \psi^{\theta}-\partial_{r}\left(\frac{\psi^{\theta}}{r}\right) \partial_{r}^{2} \psi^{\theta}\right) r d r d z \\
=\int_{-\infty}^{\infty} \int_{0}^{\infty}\left(\left[\partial_{r}\left(\frac{\psi^{\theta}}{r}\right)\right]^{2} r+\frac{\psi^{\theta}}{r} \partial_{r}^{2} \psi^{\theta}\right. \\
\left.\quad-\frac{1}{2} \partial_{r}\left[\left|\partial_{r} \psi^{\theta}\right|^{2}+\left|\partial_{z} \psi^{\theta}\right|^{2}\right]\right) d r d z \\
=\int_{-\infty}^{\infty} \int_{0}^{\infty}\left(\left[\partial_{r}\left(\frac{\psi^{\theta}}{r}\right)\right]^{2} r-\partial_{r}\left(\frac{\psi^{\theta}}{r}\right) \partial_{r}\left(\frac{\psi^{\theta}}{r} r\right)\right) d r d z \\
+\int_{-\infty}^{\infty}\left(\frac{1}{2}\left|\partial_{r} \psi^{\theta}\right|^{2}-\frac{\psi^{\theta}}{r} \partial_{r} \psi^{\theta}\right)_{r=0+} d z \\
=-\frac{1}{2} \int_{-\infty}^{\infty} \int_{0}^{\infty} \partial_{r}\left(\frac{\psi^{\theta}}{r}\right)^{2} d r d z \\
\quad+\int_{-\infty}^{\infty}\left(\frac{1}{2}\left|\partial_{r} \psi^{\theta}\right|^{2}-\frac{\psi^{\theta}}{r} \partial_{r} \psi^{\theta}\right)_{r=0+} d z \\
=\left.\frac{1}{2} \int_{-\infty}^{\infty}\left(\frac{\psi^{\theta}}{r}-\partial_{r} \psi^{\theta}\right)^{2}\right|_{r=0+} d z=0,
\end{aligned}
$$

where $\left.f\right|_{r=0+}=\lim _{r \rightarrow 0+} f(r)$. This proves (3.11). The second inequality of (3.13) follows immediately from (3.11).

It remains to prove the first inequality of (3.13). Using (3.20), (3.17), and an argument similar to that of proving (3.23), we obtain

$$
\begin{gathered}
\int_{-\infty}^{\infty} \int_{0}^{\infty}\left(\left|\nabla_{y} u_{1}\right|^{2}+2\left|\Delta_{y} \psi_{1}\right|^{2}\right) r^{3} d r d z-\int_{-\infty}^{\infty} \int_{0}^{\infty}\left|\nabla_{x} \mathbf{u}\right|^{2} r d r d z \\
=\int_{-\infty}^{\infty} \int_{0}^{\infty}\left(\left|\partial_{r}^{2} \psi^{\theta}\right|^{2}+\left|\partial_{z}^{2} \psi^{\theta}\right|^{2}+2\left|\partial_{r z}^{2} \psi^{\theta}\right|^{2}+2\left[\partial_{z}\left(\frac{\psi^{\theta}}{r}\right)\right]^{2}\right. \\
+13\left[\partial_{r}\left(\frac{\psi^{\theta}}{r}\right)\right]^{2}-10 \partial_{z}\left(\frac{\psi^{\theta}}{r}\right) \partial_{r z}^{2} \psi^{\theta} \\
\left.-10 \partial_{r}\left(\frac{\psi^{\theta}}{r}\right) \partial_{r}^{2} \psi^{\theta}\right) r d r d z \\
=\int_{-\infty}^{\infty} \int_{0}^{\infty}\left(\left|\partial_{r}^{2} \psi^{\theta}\right|^{2}+\left|\partial_{z}^{2} \psi^{\theta}\right|^{2}+2\left|\partial_{r z}^{2} \psi^{\theta}\right|^{2}+2\left[\partial_{z}\left(\frac{\psi^{\theta}}{r}\right)\right]^{2}\right) r d r d z \\
+3 \int_{-\infty}^{\infty} \int_{0}^{\infty}\left[\partial_{r}\left(\frac{\psi^{\theta}}{r}\right)\right]^{2} r d r d z \geq 0 .
\end{gathered}
$$

This completes the proof of Proposition 3.3. 


\section{Generalized Model Equations in an Arbitrary Space Dimension}

In this section, we generalize our three-dimensional model (3.1) to an arbitrary space dimension. On one hand, the generalized model improves our understanding of the original three-dimensional model. On the other hand, the result we obtain for the generalized model is of independent interest in itself.

Let $n \geq-1$ be an integer and denote $y=\left(y_{1}, \ldots, y_{n+1}, z\right) \in \mathbb{R}^{n+2}$. The generalized model is given by the following system of equations:

$$
\left\{\begin{array}{l}
\partial_{t} u_{1}=v \Delta_{n+2} u_{1}+2 \partial_{z} \psi_{1} u_{1} \\
\partial_{t} \omega_{1}=v \Delta_{n+2} \omega_{1}+\left(\left(u_{1}\right)^{2}\right)_{z} \\
-\Delta_{n+2} \psi_{1}=\omega_{1}
\end{array}\right.
$$

where $\Delta_{n+2}=\partial_{y_{1}}^{2}+\cdots+\partial_{y_{n+1}}^{2}+\partial_{z}^{2}$.

The three-dimensional model we introduced in the previous section corresponds to the case of $n=3$, which can also be considered as a model in five space dimensions. It is easy to check that (3.14) and (3.15) are still valid for the generalized system (4.1). Thus we have the same energy identity

$$
\frac{1}{2} \frac{d}{d t} \int_{\mathbb{R}^{n+2}}\left(u_{1}^{2}+2\left|\nabla_{y} \psi_{1}\right|^{2}\right) d y+v \int_{\mathbb{R}^{n+2}}\left(\left|\nabla_{y} u_{1}\right|^{2}+2\left|\Delta_{y} \psi_{1}\right|^{2}\right) d y=0 .
$$

The initial condition for the generalized model (4.1) is of the form

$$
u_{1}(0, y)=u_{10}(y), \quad \omega_{1}(0, y)=\omega_{10}(y), \quad \psi_{1}(0, y)=\psi_{10}(y) .
$$

Based on the balance between the diffusion term and the nonlinear vortexstretching term, we can classify the generalized model as subcritical if $n<2$, critical if $n=2$, and supercritical if $n>2$. Like the three-dimensional NavierStokes equations, our three-dimensional model, which corresponds to $n=3$, is supercritical. In the following, we prove the global regularity of the generalized model for the subcritical and the critical cases.

THEOREM 4.1 Assume that $-1 \leq n \leq 2$ and $\left(u_{10}, \omega_{10}, \psi_{10}\right)$ satisfies

$$
\omega_{10}=-\Delta_{y} \psi_{10}, \quad u_{10}, \nabla \psi_{10} \in H^{1}\left(\mathbb{R}^{n+2}\right) .
$$

Then there exists a globally smooth solution to system (4.1) with the initial data (4.3).

PROOF: We first consider the critical case of $n=2$. Applying $\nabla_{y}$ to the first equation in (4.1) and then taking the $L^{2}$ inner product of the resulting equation 
with $\nabla_{y} u_{1}$, we have

$$
\begin{aligned}
& \frac{1}{2} \frac{d}{d t} \int_{\mathbb{R}^{4}}\left|\nabla_{y} u_{1}\right|^{2} d y+v \int_{\mathbb{R}^{4}}\left|\Delta_{y} u_{1}\right|^{2} d y \\
& \quad=-2 \int_{\mathbb{R}^{4}} \partial_{z} \psi_{1} u_{1} \nabla_{y}^{2} u_{1} d y \\
& \quad \leq \frac{1}{2} v\left\|\Delta_{y} u_{1}\right\|_{L^{2}\left(\mathbb{R}^{4}\right)}^{2}+\frac{2}{v}\left\|u_{1}\right\|_{L^{4}\left(\mathbb{R}^{4}\right)}^{2}\left\|\partial_{z} \psi_{1}\right\|_{L^{4}\left(\mathbb{R}^{4}\right)}^{2} \\
& \quad \leq \frac{1}{2} v\left\|\nabla_{y}^{2} u_{1}\right\|_{L^{2}\left(\mathbb{R}^{4}\right)}^{2}+\frac{C}{v}\left\|\nabla_{y} u_{1}\right\|_{L^{2}\left(\mathbb{R}^{4}\right)}^{2}\left\|\nabla_{y} \partial_{z} \psi_{1}\right\|_{L^{2}\left(\mathbb{R}^{4}\right)}^{2} \\
& \quad \leq \frac{1}{2} v\left\|\nabla_{y}^{2} u_{1}\right\|_{L^{2}\left(\mathbb{R}^{4}\right)}^{2}+\frac{C}{v}\left(\left\|\nabla_{y} u_{1}\right\|_{L^{2}\left(\mathbb{R}^{4}\right)}^{4}+\left\|\omega_{1}\right\|_{L^{2}\left(\mathbb{R}^{4}\right)}^{4}\right),
\end{aligned}
$$

where we have used $\left\|u_{1}\right\|_{L^{4}\left(\mathbb{R}^{4}\right)} \leq C\left\|\nabla_{y} u_{1}\right\|_{L^{2}\left(\mathbb{R}^{4}\right)}$, the Sobolev embedding inequality.

Similarly, taking the $L^{2}$ inner product of the second equation in (4.1) with $\omega_{1}$ and performing integration by parts, we have

$$
\begin{aligned}
\frac{1}{2} & \frac{d}{d t} \int_{\mathbb{R}^{4}}\left|\omega_{1}\right|^{2} d y+v \int_{\mathbb{R}^{4}}\left|\nabla_{y} \omega_{1}\right|^{2} d y \\
\leq & \frac{1}{2} v\left\|\nabla_{y} \omega_{1}\right\|_{L^{2}\left(\mathbb{R}^{4}\right)}^{2}+\frac{2}{v}\left\|u_{1}\right\|_{L^{4}\left(\mathbb{R}^{4}\right)}^{4} \\
\leq & \frac{1}{2} v\left\|\nabla_{y} \omega_{1}\right\|_{L^{2}\left(\mathbb{R}^{4}\right)}^{2}+\frac{C}{v}\left\|\nabla_{y} u_{1}\right\|_{L^{2}\left(\mathbb{R}^{4}\right)}^{4} .
\end{aligned}
$$

Combining (4.4)-(4.5) with the basic energy identity (4.2), we arrive at the a priori estimate

$$
\begin{aligned}
& \int_{\mathbb{R}^{4}}\left(\left|\nabla_{y} u_{1}\right|^{2}+\left|\omega_{1}\right|^{2}\right) d y+2 v \int_{0}^{t} \int_{\mathbb{R}^{4}}\left(\left|\Delta_{y} u_{1}\right|^{2}+\left|\nabla_{y} \omega_{1}\right|^{2}\right) d y d s \\
& \leq \int_{\mathbb{R}^{4}}\left(\left|\nabla_{y} u_{10}\right|^{2}+\left|\omega_{10}\right|^{2}\right) d y \exp \left\{\frac{C}{v} \int_{0}^{t} \int_{\mathbb{R}^{4}}\left(\left|\nabla_{y} u_{1}\right|^{2}+\left|\omega_{1}\right|^{2}\right) d y d t\right\} \\
& \leq \int_{\mathbb{R}^{4}}\left(\left|\nabla_{y} u_{10}\right|^{2}+\left|\omega_{10}\right|^{2}\right) d y \exp \left\{\frac{C}{v^{2}}\left(\left\|\left(u_{10}, \nabla_{y} \psi_{10}\right)\right\|_{L^{2}\left(\mathbb{R}^{4}\right)}^{2}\right\} .\right.
\end{aligned}
$$

Based on the above a priori estimate, we can easily prove the global regularity of the solution in any high-order norm for all $t>0$. 
The case of $n=1$ can be proved similarly. The a priori estimate now reads

$$
\begin{gathered}
\int_{\mathbb{R}^{3}}\left(\left|\nabla_{y} u_{1}\right|^{2}+\left|\omega_{1}\right|^{2}\right) d y+2 v \int_{0}^{t} \int_{\mathbb{R}^{3}}\left(\left|\Delta_{y} u_{1}\right|^{2}+\left|\nabla_{y} \omega_{1}\right|^{2}\right) d y d s \leq \\
\int_{\mathbb{R}^{3}}\left(\left|\nabla_{y} u_{10}\right|^{2}+\left|\omega_{10}\right|^{2}\right) d y \exp \left\{\frac{C t^{1 / 2}}{v^{3 / 2}}\left(\left\|\left(u_{10}, \nabla_{y} \psi_{10}\right)\right\|_{L^{2}\left(\mathbb{R}^{3}\right)}\right)\right\} .
\end{gathered}
$$

The cases of $n=0$ and $n=-1$ are straightforward. We omit the proof here.

\section{A Special One-Dimensional Model}

It is also interesting to consider the special case when the solution develops a large gradient along the $z$-direction and the derivative along the $r$-direction is relatively smooth. In this case, the Laplacian operator becomes $z$-dominated and can be approximated locally by the one-dimensional Laplacian along the $z$-direction. This gives rise to the following one-dimensional model, which corresponds to the case of $n=-1$ in our generalized system:

$$
\begin{aligned}
\partial_{t} u_{1} & =v \partial_{z}^{2} u_{1}+2 \partial_{z} \psi_{1} u_{1}, \\
\partial_{t} \omega_{1} & =v \partial_{z}^{2} \omega_{1}+\left(\left(u_{1}\right)^{2}\right)_{z}, \\
-\partial_{z}^{2} \psi_{1} & =\omega_{1} .
\end{aligned}
$$

Further, we introduce a new variable $v_{1}=\partial_{z} \psi_{1}$. By integrating equation (5.2) with respect to $z$, we obtain an evolution equation for $v_{1}$ as follows:

$$
\partial_{t} v_{1}=v \partial_{z}^{2} v_{1}-u_{1}^{2}+c(t)
$$

where $c(t)$ is an integration constant. If we consider a periodic boundary condition in $z$ with period 1 , we can determine $c$ explicitly by enforcing $\int_{0}^{1} v_{1} d z=0$ since $v_{1}=\partial_{z} \psi_{1}$. This gives $c(t)=\int_{0}^{1} u_{1}^{2}(t, z) d z$. Then we obtain an equivalent system for $u_{1}$ and $v_{1}$ as follows:

$$
\left\{\begin{array}{l}
\partial_{t} u_{1}=v \partial_{z}^{2} u_{1}+2 u_{1} v_{1}, \\
\partial_{t} v_{1}=v \partial_{z}^{2} v_{1}-u_{1}^{2}+c(t),
\end{array}\right.
$$

where $c(t)=\int_{0}^{1} u_{1}^{2}(t, z) d z$. It is easy to show that we have

$$
\frac{1}{2} \frac{d}{d t}\left(u_{1}^{2}+2 v_{1}^{2}\right)=v\left(u_{1} \partial_{z}^{2} u_{1}+2 v_{1} \partial_{z}^{2} v_{1}\right)+2 c(t) v_{1} .
$$


Using (5.6) and the fact that $\int_{0}^{1} v_{1}(t, z) d z=0$, we have

$$
\begin{aligned}
\int_{0}^{1}( & \left.u_{1}^{2}(t, z)+2 v_{1}^{2}(t, z)\right) d z \\
= & -v \int_{0}^{t} \int_{0}^{1}\left(\left(\partial_{z} u_{1}\right)^{2}+\left(\partial_{z} v_{1}\right)^{2}\right) d z d t \\
& +\int_{0}^{1}\left(u_{1}^{2}(0, z)+2 v_{1}^{2}(0, z)\right) d z
\end{aligned}
$$

This a priori estimate is sufficient to establish the global regularity of the onedimensional model for initial data $u_{1}(0, z), v_{1}(0, z) \in L^{2}[0,1]$.

An interesting feature of this one-dimensional model is that even without any diffusion, its solution still has global regularity. To see this, consider the inviscid model with $v=0$. Combining (5.6) with (5.7), we get

$$
\begin{aligned}
\frac{d}{d t}\left(u_{1}^{2}+2 v_{1}^{2}\right) & =4 c(t) v_{1} \\
& \leq\left(u_{1}^{2}+2 v_{1}^{2}\right)+2\left(\int_{0}^{1}\left(u_{1}^{2}(0, z)+2 v_{1}^{2}(0, z)\right) d z\right)^{2} .
\end{aligned}
$$

By the Gronwall inequality, we obtain the following a priori estimate:

$$
\begin{aligned}
u_{1}^{2}+2 v_{1}^{2} \leq & \left(u_{1}^{2}(0, z)+2 v_{1}^{2}(0, z)\right) \mathrm{e}^{t} \\
& +2\left(\mathrm{e}^{t}-1\right)\left(\int_{0}^{1}\left(u_{1}^{2}(0, z)+2 v_{1}^{2}(0, z)\right) d z\right)^{2} .
\end{aligned}
$$

Using the above a priori estimate, we can prove global regularity in any highorder norm. Let $m \geq 1$ be any integer. We have

$$
\begin{aligned}
\frac{d}{d t} & \int_{0}^{1}\left(\left|\partial_{z}^{m} u_{1}\right|^{2}+\left|\partial_{z}^{m} v_{1}\right|^{2}\right) d z \\
& =\int_{0}^{1}\left[\partial_{z}^{m} u_{1} \partial_{z}^{m}\left(2 u_{1} v_{1}\right)-\partial_{z}^{m} v_{1} \partial_{z}^{m}\left(u_{1}^{2}\right)\right] d z \\
& \leq C\left(\left\|u_{1}\right\|_{L^{\infty}}+\left\|v_{1}\right\|_{L^{\infty}}\right) \int_{0}^{1}\left(\left|\partial_{z}^{m} u_{1}\right|^{2}+\left|\partial_{z}^{m} v_{1}\right|^{2}\right) d z
\end{aligned}
$$

Using (5.8), one obtains

$$
\begin{aligned}
& \int_{0}^{1}\left(\left|\partial_{z}^{m} u_{1}\right|^{2}+\left|\partial_{z}^{m} v_{1}\right|^{2}\right) d z \\
& \quad \leq \exp \left\{\int_{0}^{t} C\left(\left\|u_{1}\right\|_{L^{\infty}}+\left\|v_{1}\right\|_{L^{\infty}}\right) d s\right\} \int_{0}^{1}\left(\left|\partial_{z}^{m} u_{1}(0, z)\right|^{2}+\left|\partial_{z}^{m} v_{1}(0, z)\right|^{2}\right) d z \\
& \quad \leq \exp \left(\exp \left\{C_{\star} t\right\}\right) \int_{0}^{1}\left(\left|\partial_{z}^{m} u_{1}(0, z)\right|^{2}+\left|\partial_{z}^{m} v_{1}(0, z)\right|^{2}\right) d z
\end{aligned}
$$


where $C_{\star}$ is an absolute positive constant depending only on the $L^{2}$ norm and the $L^{\infty}$ norm of the initial data. This proves the global regularity of the onedimensional inviscid model. It is interesting to observe that the growth rate of the high-order norm is double exponential in time.

As we will see in the next section, when the solution of the original threedimensional model develops a strong layer along the $z$-direction, the solution does not become singular in a finite time. The analysis of the one-dimensional model provides us with important guidance in our computational study of possible finite time singularities.

\section{Nearly Singular Behavior of the Three-Dimensional Model}

In this section, we will present numerical results to demonstrate the near singular behavior of the three-dimensional model. We will also illustrate the mechanism that leads to the formation of a potential finite time singularity of the model and how the convection term destroys such a mechanism, preventing the formation of the potential finite time singularity in the full Navier-Stokes equations. We caution that the evidence for singularity formation of the three-dimensional model is not yet conclusive. It requires substantially higher numerical resolutions than what we have currently used to give more definitive evidence.

\subsection{Setup of the Problem}

We solve the three-dimensional model equation over a cylindrical domain: $\Omega=$ $\left\{(z, r) \mid 0 \leq z \leq \frac{1}{2}, 0 \leq r \leq 1\right\}$. Note that $\psi$ is determined only up to a constant. To define $\psi$ uniquely, we may assume that $\int_{0}^{1} \psi_{1}(z, 1, t) d z=0$ for all times. We use periodic boundary conditions along the $z$-direction with period $\frac{1}{2}$ and no-slip, no-flow boundary conditions at $r=1$, i.e., $\left.\mathbf{u}\right|_{r=1}=\mathbf{0}$, which is equivalent to

$$
\begin{gathered}
u^{r}=u^{\theta}=u^{z}=0, \\
\text { i.e., }-r \partial_{z} \psi_{1}=r u_{1}=2 \psi_{1}+r \partial_{r} \psi_{1}=0 \quad \text { on } r=1 .
\end{gathered}
$$

Since $\int_{0}^{1} \psi_{1}(z, 1, t) d z=0$, the above condition is equivalent to

$$
\left.u_{1}\right|_{r=1}=0,\left.\quad \psi_{1}\right|_{r=1}=0,\left.\quad \frac{\partial}{\partial r} \psi_{1}\right|_{r=1}=0 .
$$

Since our three-dimensional model is formulated in the stream function-vorticity setting, we need to derive a boundary condition for $\omega_{1}$. Recall that

$$
\omega_{1}=-\frac{\partial^{2}}{\partial z^{2}} \psi_{1}-\frac{\partial^{2}}{\partial r^{2}} \psi_{1}-\frac{3}{r} \frac{\partial}{\partial r} \psi_{1}
$$

Thus the no-slip, no-flow boundary condition (6.2) implies that

$$
\left.\omega_{1}\right|_{r=1}=-\left.\frac{\partial^{2}}{\partial r^{2}} \psi_{1}\right|_{r=1} .
$$


Let us discretize the domain $\Omega$ by a $N_{z} \times N_{r}$ grid. Denote $z_{j}=j h_{z}, r_{j}=j h_{r}$, with $h_{z}=1 /\left(2 N_{z}\right)$ and $h_{r}=1 / N_{r}$. Further, we denote $\left(\psi_{1}\right)_{i, j} \approx \psi_{1}\left(z_{i}, r_{j}\right)$, $\left(u_{1}\right)_{i, j} \approx u_{1}\left(z_{i}, r_{j}\right)$, and $\left(\omega_{1}\right)_{i, j} \approx \omega_{1}\left(z_{i}, r_{j}\right)$. We will discretize the threedimensional model in space by a second-order centered difference method and discretize the three-dimensional model in time by using the classical fourth-order Runge-Kutta method. To derive a discrete boundary condition for $\omega_{1}$ at $r=1$, we approximate $\left.\left(\psi_{1}\right)_{r r}\right|_{r=1}$ by a second-order finite difference method. This gives

$$
\omega_{1}\left(z_{i}, 1\right) \approx-\frac{\left(\psi_{1}\right)_{i, N r+1}-2\left(\psi_{1}\right)_{i, N r}+\left(\psi_{1}\right)_{i, N r-1}}{h_{r}^{2}} .
$$

Using the boundary condition (6.2) for the stream function at $r=1$, we conclude that $\left(\psi_{1}\right)_{i, N r}=0$. Moreover, we can approximate the second boundary condition $\left.\left(\psi_{1}\right)_{r}\right|_{r=1}=0$ by using a second-order centered difference approximation to $\left.\left(\psi_{1}\right)_{r}\right|_{r=1}$. This gives

$$
\frac{\left(\psi_{1}\right)_{i, N r+1}-\left(\psi_{1}\right)_{i, N r-1}}{h_{r}}=0,
$$

or, equivalently,

$$
\left(\psi_{1}\right)_{i, N r+1}=\left(\psi_{1}\right)_{i, N r-1} \text {. }
$$

Substituting (6.6) into (6.5), we obtain our discrete boundary condition for $\omega_{1}$ at $r=1$,

$$
\left(\omega_{1}\right)_{i, N r}=-\frac{2\left(\psi_{1}\right)_{i, N r-1}}{h_{r}^{2}} .
$$

This is the well-known Thom's boundary condition [28]. In [19], Hou and Wetton proved that the second-order finite difference approximation of the NavierStokes equations using the Thom's boundary condition converges with a secondorder accuracy.

To summarize, the discrete no-slip, no-flow boundary at $r=1$ is given by

$$
\left(u_{1}\right)_{i, N_{r}}=0, \quad\left(\psi_{1}\right)_{i, N_{r}}=0, \quad\left(\omega_{1}\right)_{i, N_{r}}=-\frac{2\left(\psi_{1}\right)_{i, N r-1}}{h_{r}^{2}} .
$$

The periodic boundary condition along the $z$-direction and the boundary condition (6.8) at $r=1$ plus the initial condition completely determine the dynamic evolution of the discretized system of our three-dimensional model.

The initial condition we consider in our numerical computations is given by

$$
\begin{aligned}
& u_{1}(z, r, 0)=(1+\sin (4 \pi z))\left(r^{2}-1\right)^{20}\left(r^{2}-1.2\right)^{30}, \\
& \psi_{1}(z, r, 0)=0 \\
& \omega_{1}(z, r, 0)=0 .
\end{aligned}
$$

Note that the compatibility condition (3.5) implies that $u_{1}, \psi_{1}$, and $\omega_{1}$ must be even functions of $r$. The above initial condition satisfies this constraint and the no-slip, no-flow boundary condition. We choose the initial condition in such a way that its 
support is centered around $r=0$ and the solution is nearly flat away from $r=0$. Since the initial value for $\psi_{1}$ is identically equal to 0 , the velocity fields along the radial and the axial directions are all 0 . Thus, the motion is induced by the swirling component of the velocity field. Further, we note that the swirling component of the velocity field is always nonnegative since its initial value is nonnegative.

We have used both a uniform mesh and an adaptive mesh in our computations. Since the solution eventually becomes singular at $r=0$ and is very smooth and nearly flat away from $r=0$, we use the following coordinate transformation along the $r$-direction to achieve the adaptivity:

$$
r=f(\alpha) \equiv \alpha-\frac{0.9 \sin (\pi \alpha)}{\pi} .
$$

This transformation gives a change of variables from $r$ to $\alpha$ via $r=f(\alpha)$. Note that $f$ maps the unit interval $[0,1]$ into itself and is a smooth transformation. Moreover, the derivative of the map is given by

$$
f_{\alpha}=1-0.9 \cos (\pi \alpha) \geq 0.1 \text {. }
$$

The smallest value of $f_{\alpha}$ is achieved at $\alpha=0$ with $f_{\alpha}(0)=0.1$. If we use a uniform mesh in $\alpha$ with a mesh size $h_{r}$, then the above transformation will produce an adaptive mesh in the original $r$-space with the smallest mesh size concentrated near $r=0$. The mesh size near $r=0$ is proportional to $h_{r} / 10$. Thus, we gain an adaptivity factor of $\frac{1}{10}$ near $r=0$.

The advantage of using this mesh adaptivity is that the mesh map is very smooth and is time independent. We do not introduce mesh adaptivity along the $z$-direction because we would like to use the Fourier transform along it for the discrete elliptic equation for the stream function. After applying the Fourier transform along the $z$-direction, the discrete system for $\psi_{1}$ becomes a tridiagonal system for each wave number, which can be inverted directly. This gives rise to a very efficient elliptic solver for the stream function $\psi_{1}$.

We have performed a detailed resolution study using both the uniform mesh and the above adaptive mesh. We find that the above adaptive mesh works extremely well. Since the problem is three-dimensional, the adaptivity along the $r$-direction gives a factor of $10^{2}$ savings. In the inviscid computation, which is more singular than the viscous case, the largest resolution we use is $N_{z}=8192$ and $N_{r}=800$ with a time step of $\Delta t=1.25 \times 10^{-7}$. This provides an effective resolution of $8192^{3}$ for the corresponding three-dimensional problem with a uniform mesh. The total number of time steps we run is over a hundred thousand. Without taking advantage of the axisymmetry of the solution and the mesh adaptivity, we would not have been able to afford to perform computations using this high resolution.

\subsection{Nearly Singular Behavior of the Solution at an Early Time}

We first study the nearly singular behavior of the solution at an early time. The initial condition we consider is designed in such a way that it has a maximal probability of producing a finite time singularity for the corresponding inviscid model. 


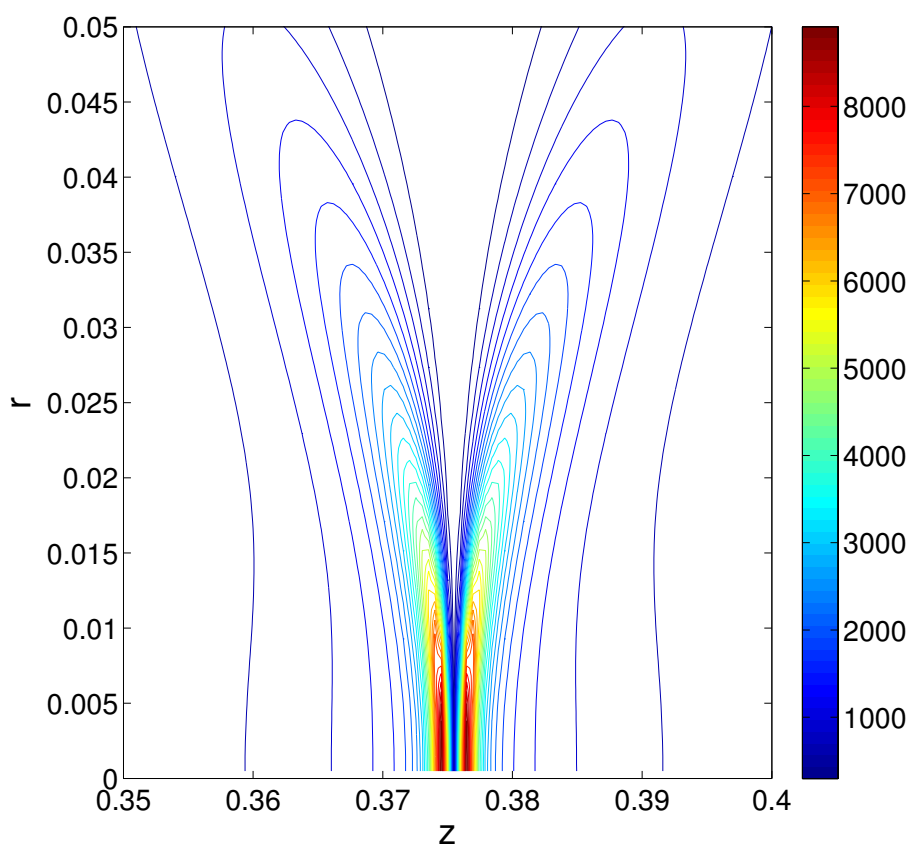

FIGURE 6.1. Closeup view of $u_{1}$-contour at $t=0.0177$ using a uniform mesh, $N_{z}=2048, N_{r}=2048, \Delta t=10^{-6}, v=0$.

A key property of this initial condition is that $u_{1}$ is nonnegative and vanishes at $z=0.375$. From the model equation for $u_{1}$ in (3.1) in the inviscid case $v=0$, we can see that $u_{1}$ remains nonnegative and vanishes at $z=0.375$ for all times. For this particular initial condition, the solution produces two large focusing centers dynamically, one to the left of $z=0.375$, and another to the right of $z=0.375$. As time increases, the axial velocity field near $r=0$ generated by this initial condition becomes large and positive for $z<0.375$ and negative for $z>0.375$. As a result, the two focusing centers are attracted toward the plane of $z=0.375$ and may experience a head-on collision in a finite time. The potential collision of the two focusing centers is a perfect candidate for generating a finite time singularity at $z=0.375$. Indeed, we find from our computations that the two focusing centers are attracted to the plane of $z=0.375$ and develop a thin layer parallel to the $r$-axis. By $t=0.0177$, the two focusing centers almost collide, and the solution becomes severely squeezed along the $z$-direction; see Figure 6.1.

We emphasize that sufficient resolution is necessary to resolve this nearly singular behavior of the solution. It is difficult to resolve the thin-layer structure of the solution of the inviscid model around $t=0.0177$. We had originally used a uniform mesh to solve the three-dimensional model equation up to $N_{z} \times N_{r}=$ 


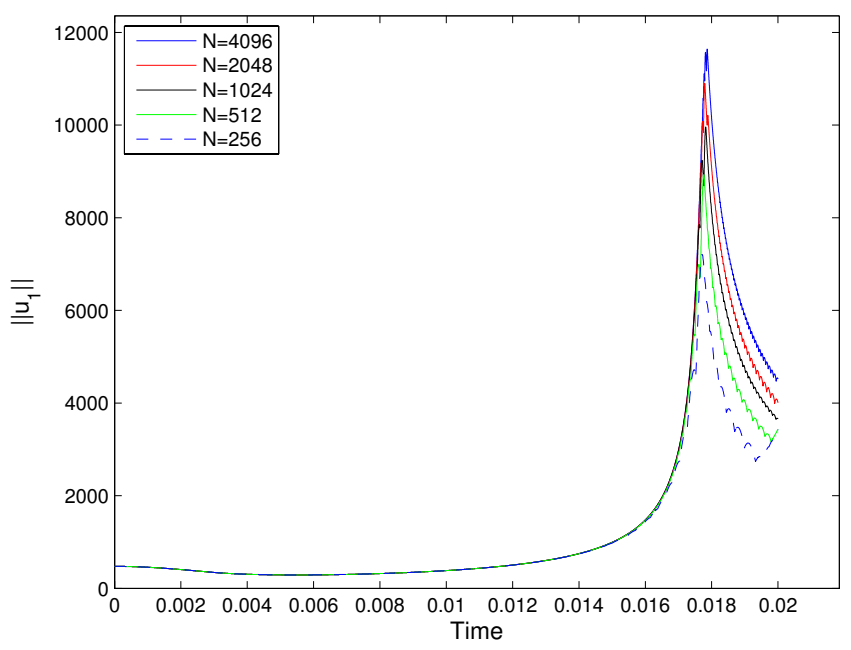

FIGURE 6.2. Maximum of $u_{1}$ in time for the inviscid model computed by uniform meshes $N_{z}=N_{r}=256$ (dashed blue), $N_{z}=N_{r}=512$ (green), and $N_{z}=N_{r}=1024$ (black), and by adaptive meshes $N_{z} \times$ $N_{r}=2048 \times 256$ (red) and $N_{z} \times N_{r}=4096 \times 400$ (blue), $v=0$. The time steps used are $\Delta t=4 \times 10^{-6}, 2 \times 10^{-6}, 10^{-6}, 5 \times 10^{-7}$, and $2.5 \times 10^{-7}$, respectively.

$2048 \times 2048$. This level of resolution is close to the limit of our computing resources without going to a massively parallel cluster. Even with this large resolution, we find that the solution of the inviscid three-dimensional model is still not well resolved near the potential singularity time. This motivates us to switch to the adaptive mesh and concentrates our computational mesh near the most singular region of the solution, i.e., the region close to the $z$-axis. The use of an adaptive mesh enables us to increase our local effective resolution considerably.

A careful resolution study is required to fully understand whether the solution of the inviscid model will develop a finite time singularity around $t=0.0178$. With $N_{z}=256,512$, and 1024, we find that the solution experiences rapid growth around $t=0.0178$; see Figure 6.2. This seems to indicate that the solution may become singular around $t=0.0178$. On the other hand, with even higher resolution $\left(N_{z} \geq 2048\right)$, we find that the growth rate of the solution seems to slow down.

If there were a true singularity around $t=0.0178$, the time at which $\left\|u_{1}\right\|_{\infty}$ achieves its maximum should have occurred earlier as we increased the resolutions. But this was not the case. We conjecture that this may be a faux singularity. As we discussed earlier, the solution becomes severely flattened and $z$-dominant locally near the region of maximum of $u_{1}$. In the region where $u_{1}$ is comparable to the 
maximum of $u_{1}$, the three-dimensional model can be approximated to the leading order by the corresponding one-dimensional model along the $z$-direction. As we showed in Section 5, the solution of the one-dimensional model cannot blow up in finite time, even in the inviscid case. This seems to provide some theoretical support for our conjecture that the nearly singular behavior of the inviscid solution around $t=0.0178$ may be a faux singularity. After $t=0.0178$, the two focusing centers move away from each other and the maximum of $u_{1}$ decreases for a short time before it experiences another period of rapid growth.

We remark that even with our largest numerical resolution we may not completely resolve the nearly singular behavior near $t=0.0178$ of the inviscid solution. To demonstrate that the solution of the inviscid model is indeed smooth around $t=0.0178$, we need to perform well-resolved computations with higher resolutions than what we have used up to now.

\subsection{Nearly Singular Behavior of the Viscous Model}

We now present numerical results which show that the solution of the viscous model becomes nearly singular. We choose the viscous coefficient to be $v=0.001$ and perform a series of resolution studies using the adaptive method. We have used both uniform mesh and adaptive mesh with $N_{z}$ ranging from 256 to 4096 . Below we present the computational results obtained by using the adaptive mesh with the highest resolution $N_{z}=4096, N_{r}=400$, and $\Delta t=2.5 \times 10^{-7}$. We will also perform a resolution study to demonstrate that our computations are well-resolved.

\section{Numerical Evidence for a Potential Finite-Time Singularity}

From our analytical study of the three-dimensional model, it follows by using a standard energy estimate that if $u_{1}$ is bounded, then the solution of the viscous three-dimensional model cannot blow up in a finite time. Thus it is sufficient to monitor the growth of $\left\|u_{1}\right\|_{\infty}$ in time. We will present numerical evidence which seems to support that $u_{1}$ may develop a potential finite time singularity for the initial condition we consider. The nature of this potential singularity and the mechanism for generating this potential singularity will be analyzed in a later subsection.

In Figure 6.3 we plot the maximum of $u_{1}$ in time over the time interval [0, 0.021] using the adaptive mesh method with $N_{z}=4096$ and $N_{r}=400$. The time step is chosen to be $\Delta t=2.5 \times 10^{-7}$. We can see that $\left\|u_{1}\right\|_{\infty}$ experiences a very rapid growth in time after $t=0.02$. In Figure 6.3 (bottom plot), we also plot $\log \left(\log \left(\left\|u_{1}\right\|_{\infty}\right)\right)$ as a function of time. We can clearly see that $\left\|u_{1}\right\|_{\infty}$ grows

much faster than double exponential in time, which implies that the solution of our model may develop a finite time singularity. We will present more careful analysis of this potentially singular behavior later.

In Figures 6.4 through 6.6, we show a sequence of contour plots for $u_{1}$ from $t=0.014$ to $t=0.021$. At early times, we observe that the solution forms two large focusing centers of $u_{1}$ that approach each other. As this occurs, these rather localized regions are squeezed and form a thin layer parallel to the $r$-axis and with 

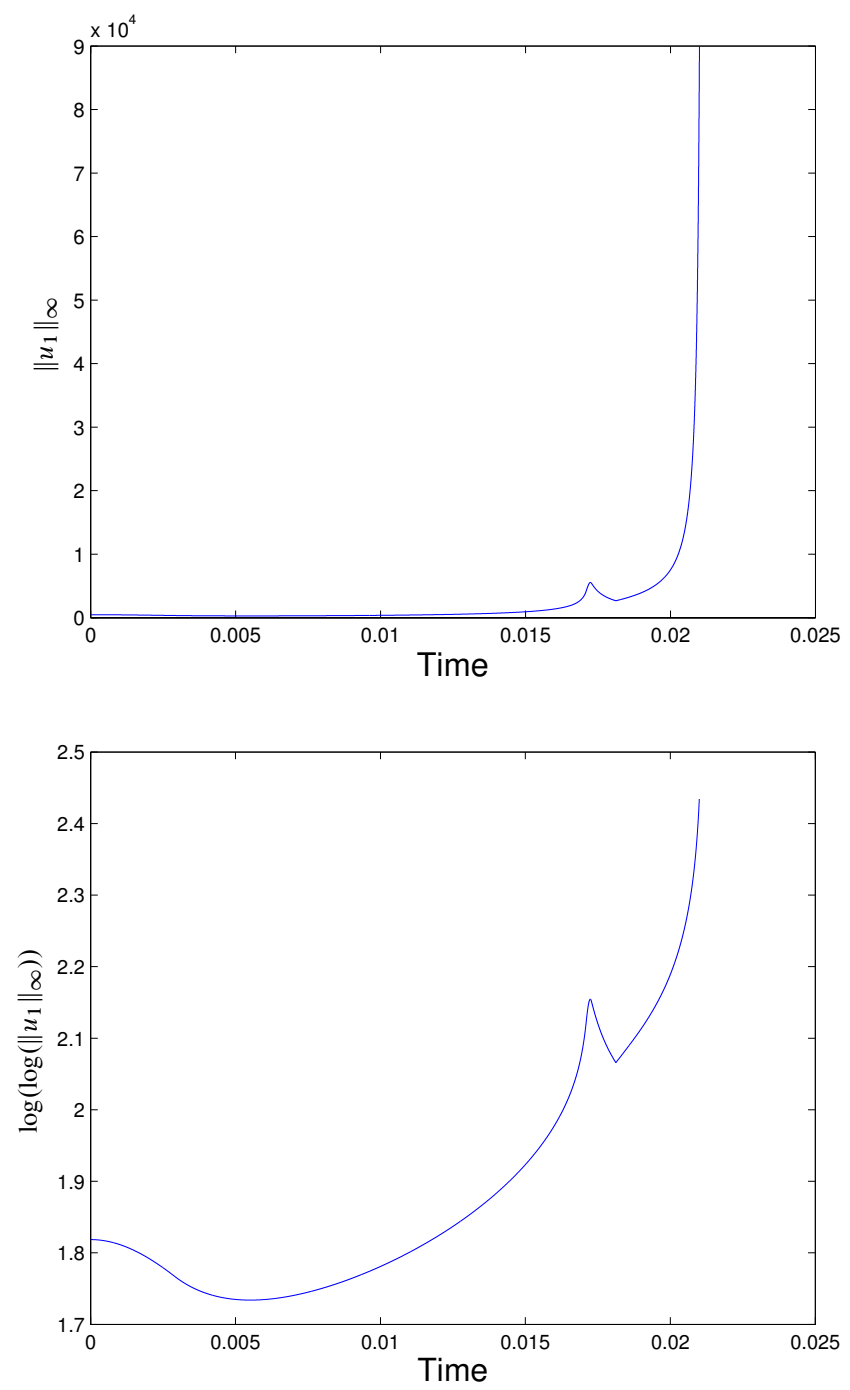

FIGURE 6.3. Left figure: $\left\|u_{1}\right\|_{\infty}$ as a function of time over the interval $[0,0.021]$. The right figure: $\log \left(\log \left(\left\|u_{1}\right\|_{\infty}\right)\right)$ as a function of time over the same interval. The solution is computed by the adaptive mesh with $N_{z}=4096, N_{r}=400, \Delta t=2.5 \times 10^{-7}, v=0.001$.

large gradients along the $z$-direction. The two focusing centers become closest around $t=0.0172$; see also Figure 6.1 for the contour of $u_{1}$ at $t=0.0177$ for the inviscid three-dimensional model.

As these regions approach each other and develop a thin layer parallel to the $r$-axis, the solution becomes locally $z$-dominant near the region where $u_{1}$ achieves 

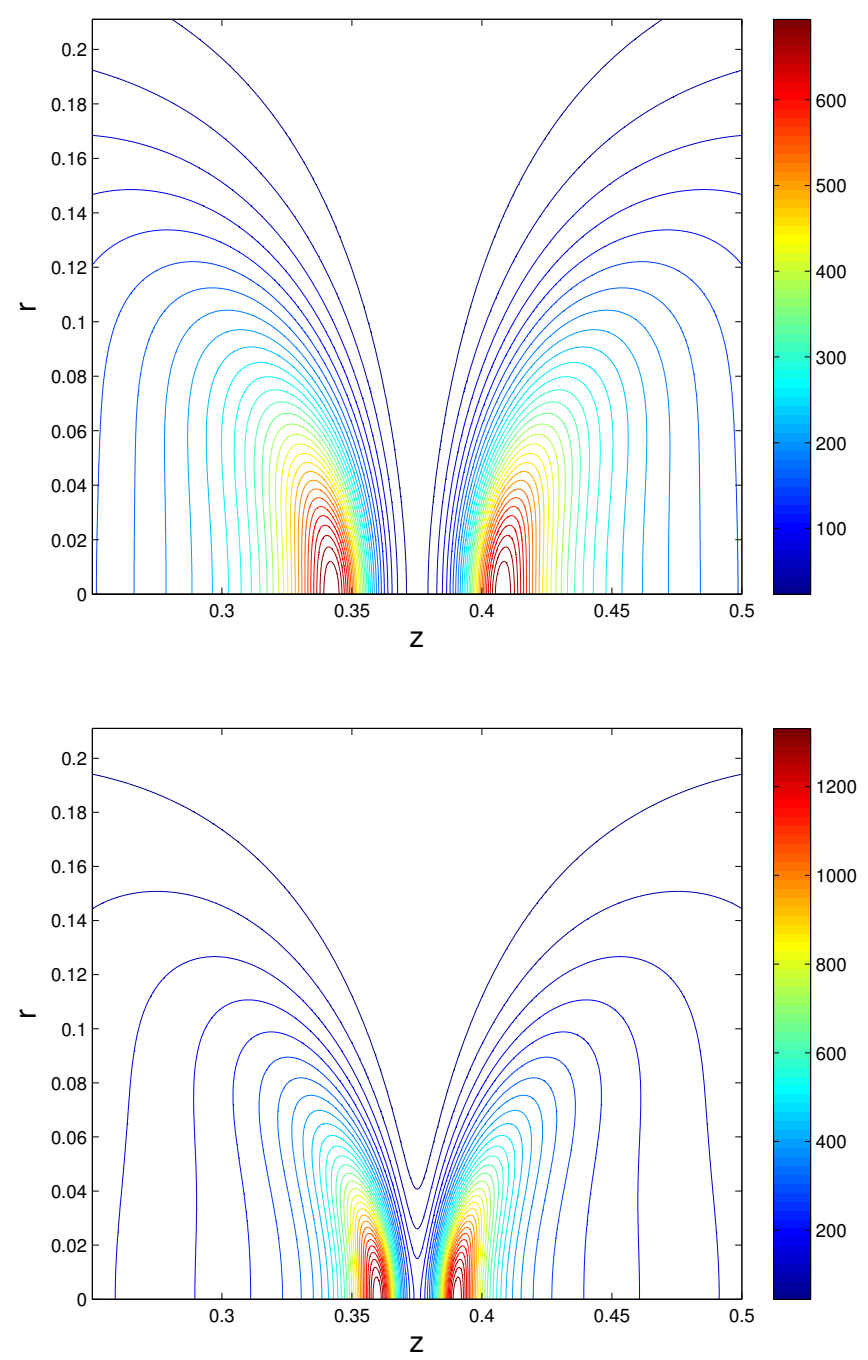

FIGURE 6.4. The contour plots of $u_{1}$ for the viscous model at $t=0.014$ (top plot) and 0.016 (bottom plot). Adaptive mesh computation with $N_{z}=4096, N_{r}=400, \Delta t=2.5 \times 10^{-7}, v=0.001$.

its maximum. In this region, the three-dimensional model can be approximated to the leading order by the corresponding one-dimensional model along the $z$ direction that we introduced in the previous section. As we have shown before, the solution of the one-dimensional model cannot blow up. After $t=0.0172$, the maximum of $u_{1}$ starts to decrease. The two focusing centers move away from each other and their supports become more isotropic. As time increases, we observe 

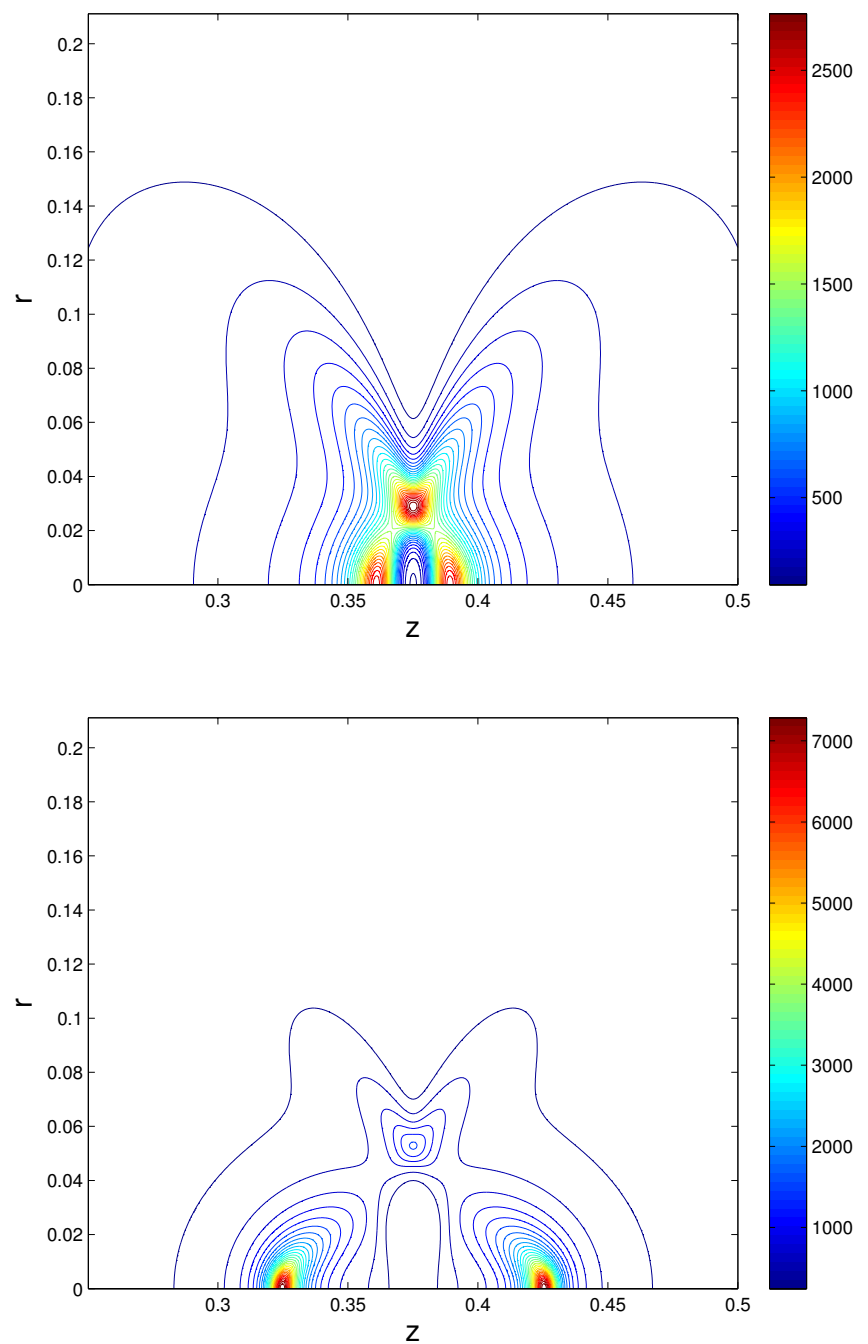

FIGURE 6.5. The contour plots of $u_{1}$ for the viscous model at $t=0.018$ (top plot) and 0.02 (bottom plot). Adaptive mesh computation with $N_{z}=4096, N_{r}=400, \Delta t=2.5 \times 10^{-7}, v=0.001$.

that there is a strong nonlinear interaction between $u_{1}$ and $\left(\psi_{1}\right)_{z}$, which is induced by the overlap between the support of the maximum of $u_{1}$ and the support of the maximum of $\left(\psi_{1}\right)_{z}$. By the support of the maximum of $u_{1}$, we mean the region in which $u_{1}$ is comparable to its maximum. The strong alignment between $u_{1}$ and $\left(\psi_{1}\right)_{z}$ near the support of the maximum of $u_{1}$ leads to a rapid growth of the solution that may become singular in a finite time. 

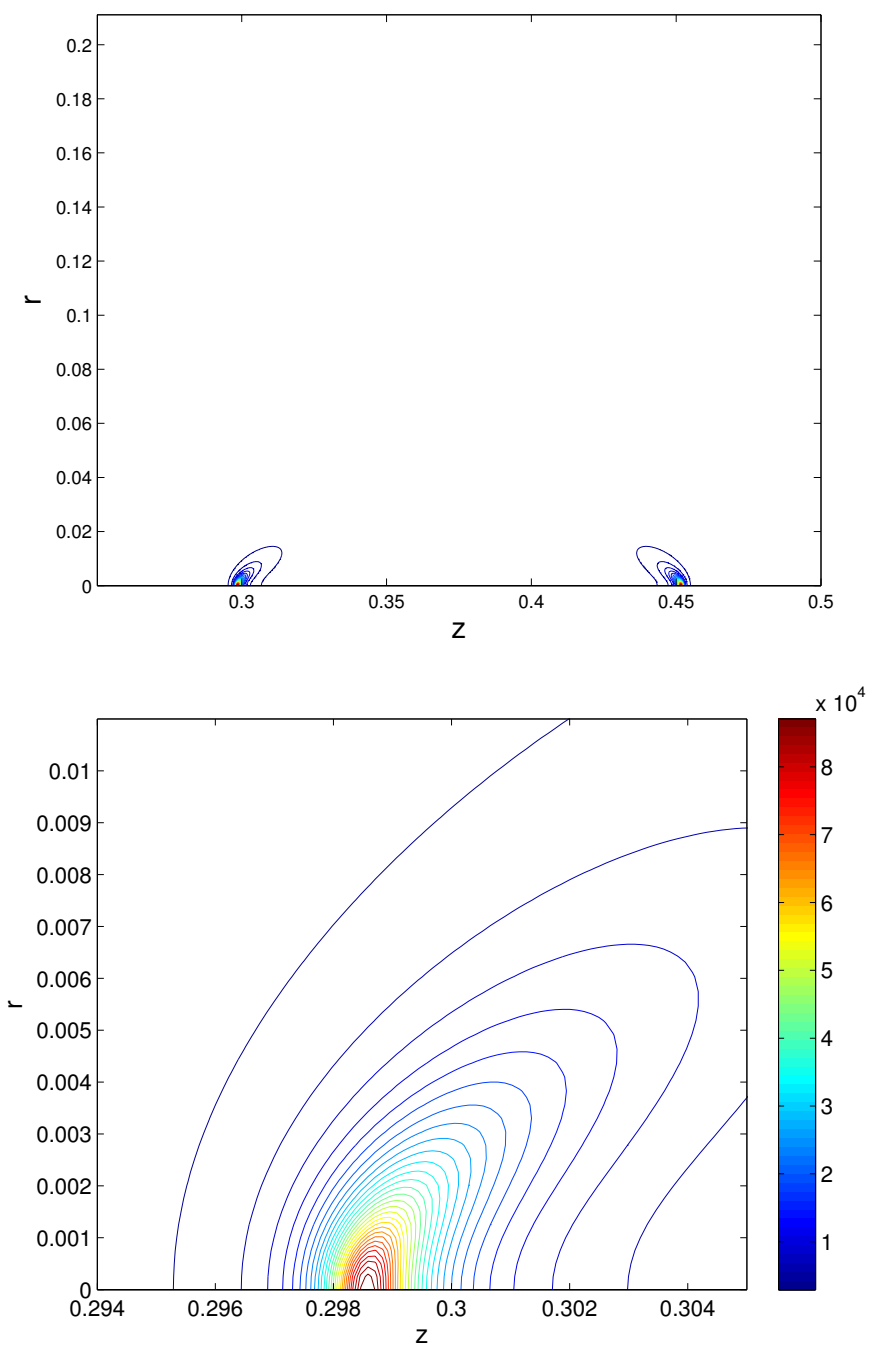

FIGURE 6.6. The contour of $u_{1}$ at $t=0.021$ (top figure) and its closeup view (bottom figure) for the viscous model computed by the adaptive mesh with $N_{z}=4096, N_{r}=400, \Delta t=2.5 \times 10^{-7}, v=0.001$.

Another important observation is that as time increases, the position at which $u_{1}$ achieves its maximum also moves toward the symmetry axis. This suggests that the potential singularity will be along the symmetry axis at the singularity time. It is easy to see from (2.7) that $\lim _{r \rightarrow 0+} u_{1}=0.5 \lim _{r \rightarrow 0+} \omega^{z}$. Thus, the blowup of $u_{1}$ characterizes the blowup of the axial vorticity $\omega^{z}$. 
Next, we perform a detailed study for the three-dimensional model and push our computations very close to the potential singularity time. We use a sequence of resolutions using both uniform and adaptive mesh. For the uniform mesh, we use resolutions for $N_{z} \times N_{r}$ ranging from $256 \times 256$ to $2048 \times 2048$ with time steps ranging from $\Delta t=5 \times 10^{-6}$ to $5 \times 10^{-7}$. For the adaptive mesh, we use $N_{z} \times N_{r}=2048 \times 256, N_{z} \times N_{r}=3072 \times 328$, and $N_{z} \times N_{r}=4096 \times 400$. The corresponding time steps for these computations are $\Delta t=10^{-6}, \Delta t=5 \times 10^{-7}$, and $\Delta t=2.5 \times 10^{-7}$, respectively. With $N_{z} \times N_{r}=4096 \times 400$, we achieve an effective resolution of $4000 \times 4000$ near the region of $r=0$ where the solution is most singular.

To obtain further evidence for a potential finite time singularity, we use a systematic singularity form fit procedure to obtain a good fit for the possible singularity of the solution. The procedure of our form fit is as follows. We look for a finite time singularity of the form

$$
\left\|u_{1}\right\|_{\infty} \approx \frac{C}{(T-t)^{\alpha}}
$$

We have tried several ways to determine the fitting parameters $T, C$, and $\alpha$. At the end, we found that the best way is to study the inverse of $\left\|u_{1}\right\|_{\infty}$ as a function of time using a sequence of numerical resolutions. This approach was used successfully before by van Dommelen and Shen in their study of the spontaneous generation of the singularity in a separating laminar boundary layer [34] (see also [20]). For each resolution, we find that the inverse of $\left\|u_{1}\right\|_{\infty}$ is almost a perfect linear function of time; see Figures 6.7 and 6.8. By using a least square fit of the inverse of $\left\|u_{1}\right\|_{\infty}$, we find that $\alpha=1$ gives the best fit. The same least square fit also determines the potential singularity time $T$ and the constant $C$. We remark that the $O(1 /(T-t))$ blowup rate of $u_{1}$, which corresponds to the blowup rate of the axial vorticity, is consistent with the nonblowup criterion of Beale-Kato-Majda type; see Section 7.

To confirm that the above procedure does indeed give a good fit for the potential singularity, we plot $\left\|u_{1}\right\|_{\infty}^{-1}$ as a function of time in Figure 6.7 (top plot). We can see that the agreement between the computed solution with $N_{z} \times N_{r}=4096 \times 400$ and the fitted solution is almost perfect. In the bottom box of Figure 6.7, we plot $\left\|u_{1}\right\|_{\infty}$ computed by our adaptive method against the form fit $C /(T-t)$ with $T=0.02109$ and $C=8.20348$. The two curves are almost indistinguishable during the final stage of the computation from $t=0.018$ to $t=0.021$.

In Figure 6.9, we also plot $\left\|\psi_{1 z}\right\|_{\infty}^{-1}$ as a function of time. We observe that $\left\|\psi_{1 z}\right\|_{\infty}^{-1}$ decays almost linearly in time with a logarithmic correction. The agreement between the computed solution with $N_{z} \times N_{r}=4096 \times 400$ and the fitted solution is excellent. In the same figure (bottom plot), we compare $\left\|\psi_{1 z}\right\|_{\infty}$ with the fitted solution. Again, we observe very good agreement between the two curves.

We further investigate the potential singular behavior of the solution by using a sequence of resolutions to study the limiting behavior of the computed solution as 

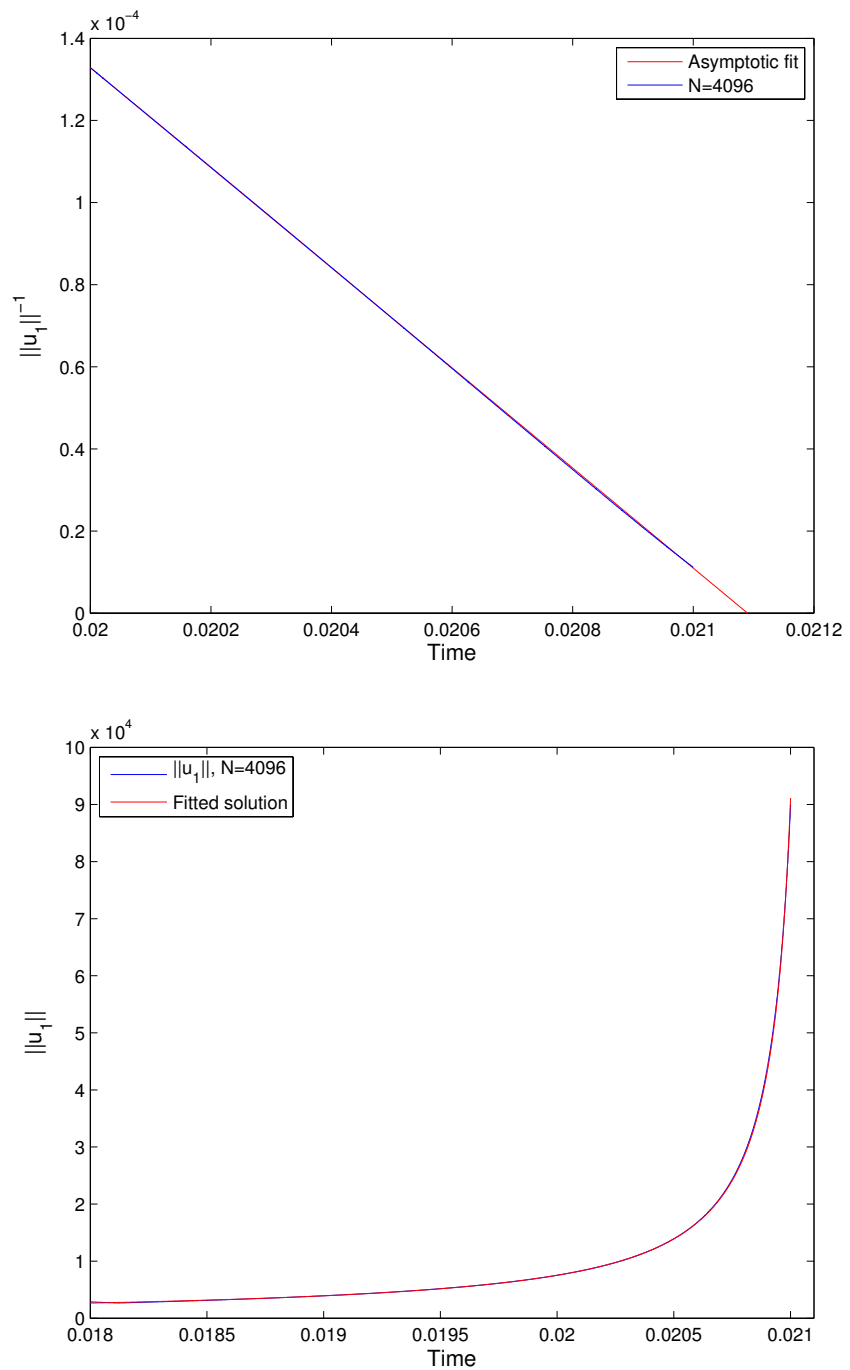

FIGURE 6.7. Top plot: The inverse of $\left\|u_{1}\right\|_{\infty}$ (blue) versus the asymptotic fit (red) for the viscous model. Right plot: $\left\|u_{1}\right\|_{\infty}$ (blue) versus the asymptotic fit (red). The asymptotic fit is of the form $\left\|u_{1}\right\|_{\infty}^{-1} \approx \frac{T-t}{C}$ with $T=0.02109$ and $C=8.20348$. The solution is computed by adaptive mesh with $N_{z}=4096, N_{r}=400, \Delta t=2.5 \times 10^{-7}$, $v=0.001$.

we refine our resolutions. The space resolutions we use are $N_{z} \times N_{r}=1024 \times 128$, $2048 \times 256,3072 \times 328$, and $4096 \times 400$, respectively. The corresponding time steps are $\Delta t=10^{-6}, 5 \times 10^{-7}, 3.625 \times 10^{-7}$, and $2.5 \times 10^{-7}$, respectively. For each resolution, we obtain an optimal least square fit of the singularity of the form 


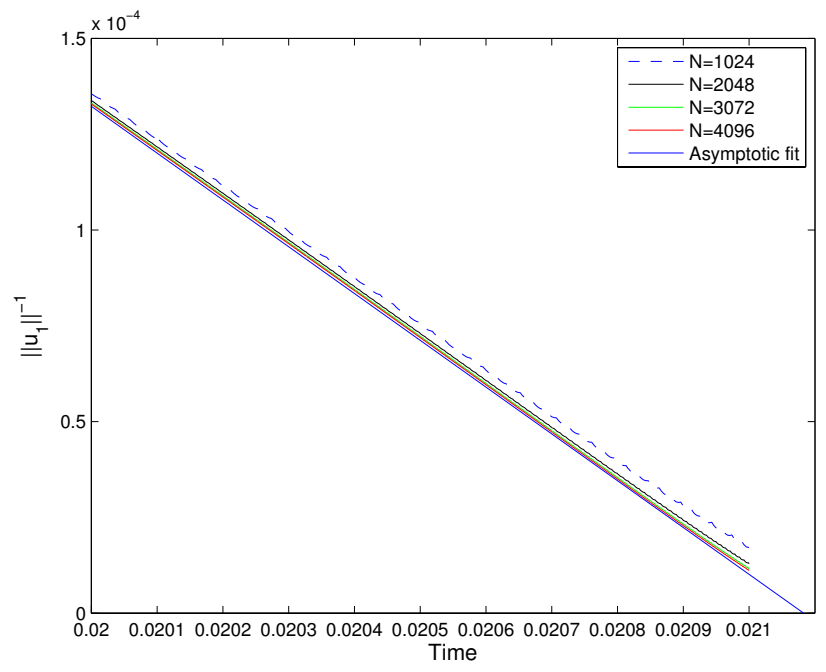

FIGURE 6.8. The inverse of $\left\|u_{1}\right\|_{\infty}$ in time for the viscous model. The solution is computed by adaptive mesh with $N_{z}=1024,2048,3072$, and 4096 (ordering from top to bottom in the figure), $\Delta t=10^{-6}, 5 \times$ $10^{-7}, 3.625 \times 10^{-7}$, and $2.5 \times 10^{-7}$, respectively. The last curve is the singularity fit by extrapolating the computational results obtained by $N_{z}=2048,3072$, and 4096 to infinite resolution $N_{z}=\infty$. The fitted curve is of the form $\left\|u_{1}\right\|_{\infty}^{-1} \approx \frac{T-t}{C}$, with $T=0.021083, C=8.1901$, and $v=0.001$.

$\left\|u_{1}\right\|_{\infty}^{-1} \approx(T-t) / C$. The results are summarized in Table 6.1. Based on the fitted parameters $T$ and $C$ from the three largest resolutions, we construct a second-order polynomial that interpolates $T$ and $C$ through these three data points. We then use the polynomial to extrapolate the values of $T$ and $C$ to the infinite resolution limit. The extrapolated values at $h_{z}=0$ are $T=0.021083$ and $C=8.1901$. In Figure 6.8, we plot the inverse of $\left\|u_{1}\right\|_{\infty}$ as a function of time using four different resolutions. We can see that as we refine the resolution, the computed solution converges to the extrapolated singularity limiting profile.

To illustrate the nature of the nearly singular solution, we show the threedimensional view of $u_{1}$ as a function of $r$ and $z$ in Figures 6.10 and 6.11. We also show the three-dimensional view of $w_{1}$ as a function of $r$ and $z$ in Figure 6.12. While $u_{1}$ is symmetric with respect to $z=0.375, w_{1}$ is antisymmetric with respect to $z=0.375$. We can see that the support of the solution $u_{1}$ in the most singular region is isotropic and appears to be locally self-similar. We will further investigate the local scaling property of the solution in Section 6.4. 

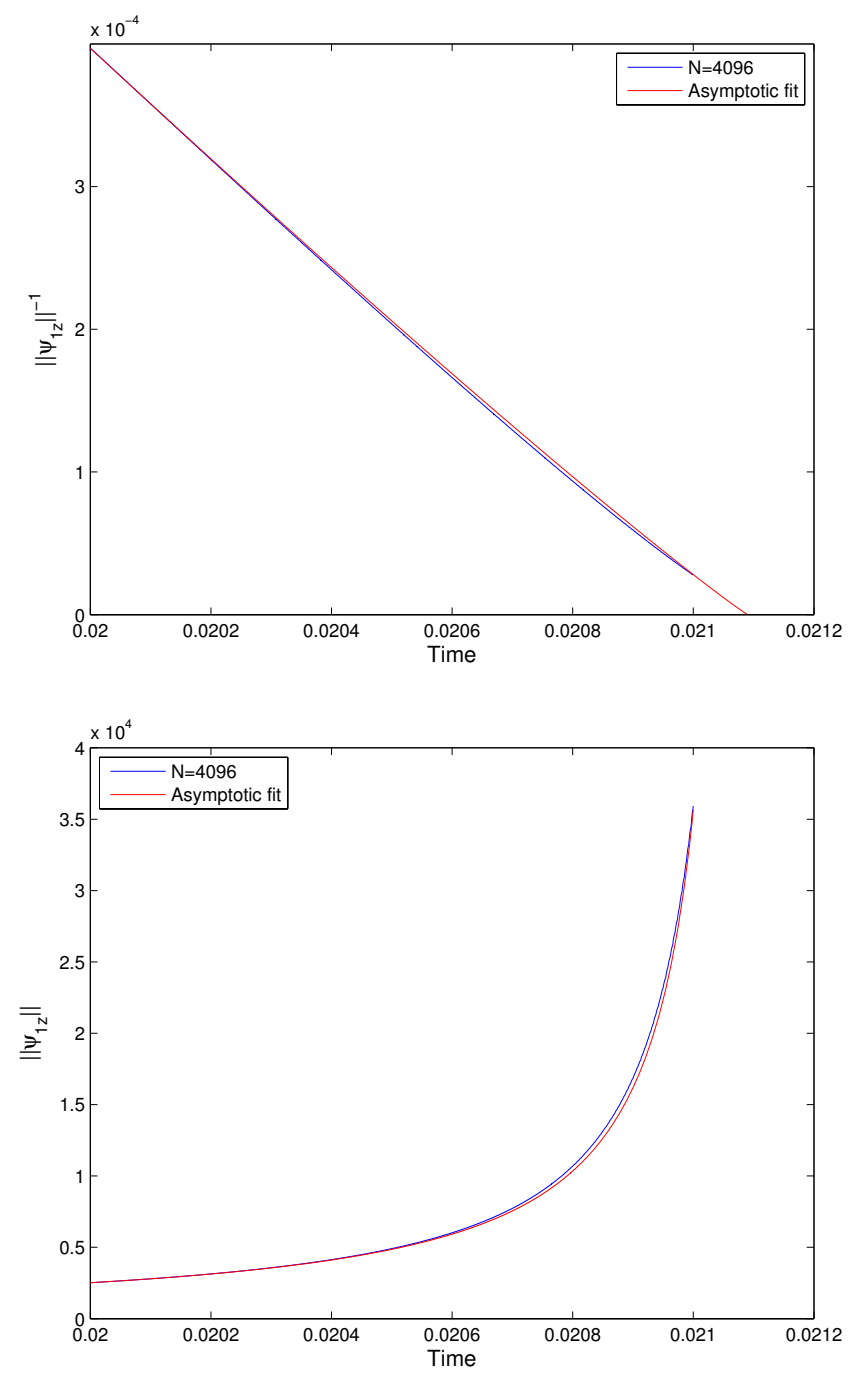

FiguRE 6.9. Top plot: The inverse of $\left\|\psi_{1 z}\right\|_{\infty}$ (blue) versus the asymptotic fit (red) for the viscous model. Bottom plot: $\left\|\psi_{1 z}\right\|_{\infty}$ (blue) versus the asymptotic fit (red). The asymptotic fit is of the form

$$
\left\|\psi_{1 z}\right\|_{\infty} \approx \frac{C(\log (1 /(T-t)))^{1 / 2}}{(T-t)}
$$

with $T=0.02109$ and $C=1.051235$. The solution is computed by adaptive mesh with $N_{z}=4096, N_{r}=400, \Delta t=2.5 \times 10^{-7}$, $v=0.001$. 


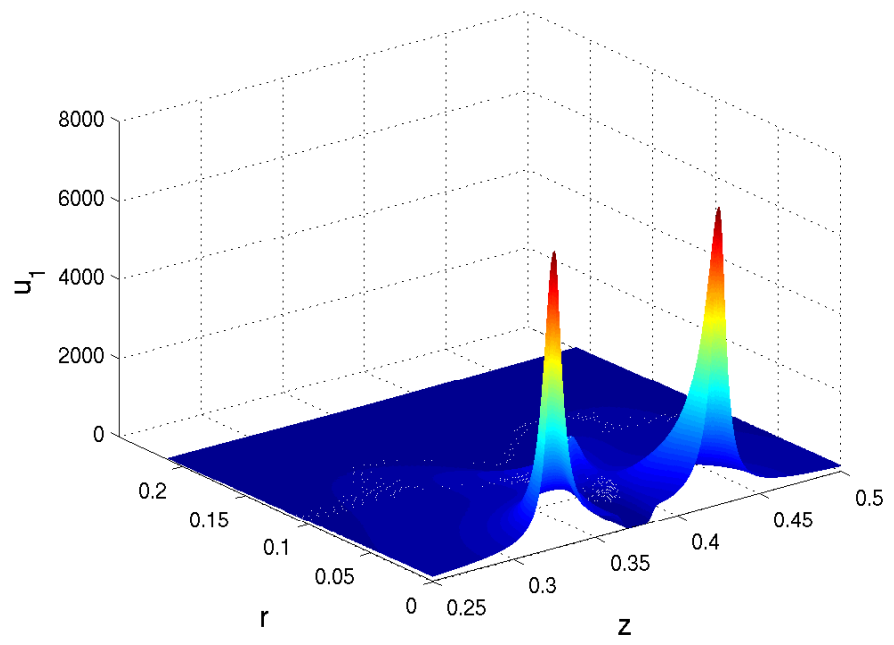

FIGURE 6.10. The three-dimensional view of $u_{1}$ at $t=0.02$ for the viscous model computed by the adaptive mesh with $N_{z}=4096, N_{r}=$ $400, \Delta t=2.5 \times 10^{-7}, v=0.001$.

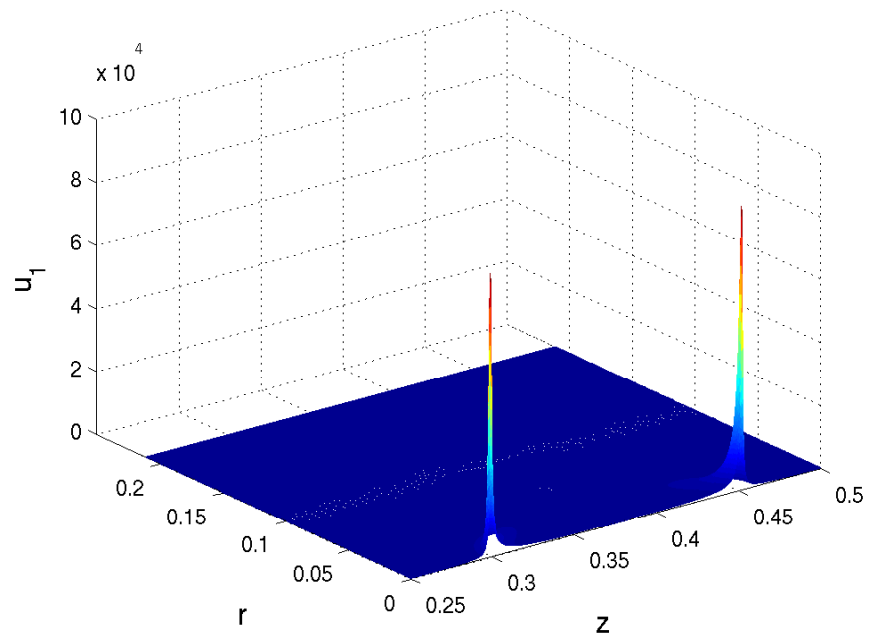

FIGURE 6.11. The three-dimensional view of $u_{1}$ at $t=0.021$ for the viscous model computed by the adaptive mesh with $N_{z}=4096, N_{r}=$ $400, \Delta t=2.5 \times 10^{-7}, v=0.001$. 


\begin{tabular}{|c|l|l|}
\hline$h_{z}$ & \multicolumn{1}{|c|}{$T$} & \multicolumn{1}{c|}{$C$} \\
\hline $1 / 2048$ & 0.02114 & 8.409 \\
\hline $1 / 4096$ & 0.0211 & 8.2237 \\
\hline $1 / 6144$ & 0.021093 & 8.20946 \\
\hline $1 / 8192$ & 0.02109 & 8.20348 \\
\hline Extrapolation to $h_{z}=0$ & 0.021083 & 8.1901 \\
\hline
\end{tabular}

TABLE 6.1. Resolution study of parameters $T$ and $C$ in the asymptotic fit for the viscous model $\left\|u_{1}\right\|_{\infty}^{-1} \approx \frac{T-t}{C}$ using different resolutions $h_{z}=$ $1 /\left(2 N_{z}\right)$. The resolutions we use in our adaptive computations are $N_{z} \times$ $N_{r}=1024 \times 128,2048 \times 256,3072 \times 328$, and $4096 \times 400$. The corresponding time steps are $\Delta t=10^{-6}, 5 \times 10^{-7}, 3.625 \times 10^{-7}$, and $2.5 \times 10^{-7}$, respectively. The last row is obtained by extrapolating the second-order polynomial that interpolates the data obtained using $h_{z}=$ $1 / 4096,1 / 6144$, and $1 / 8192$.

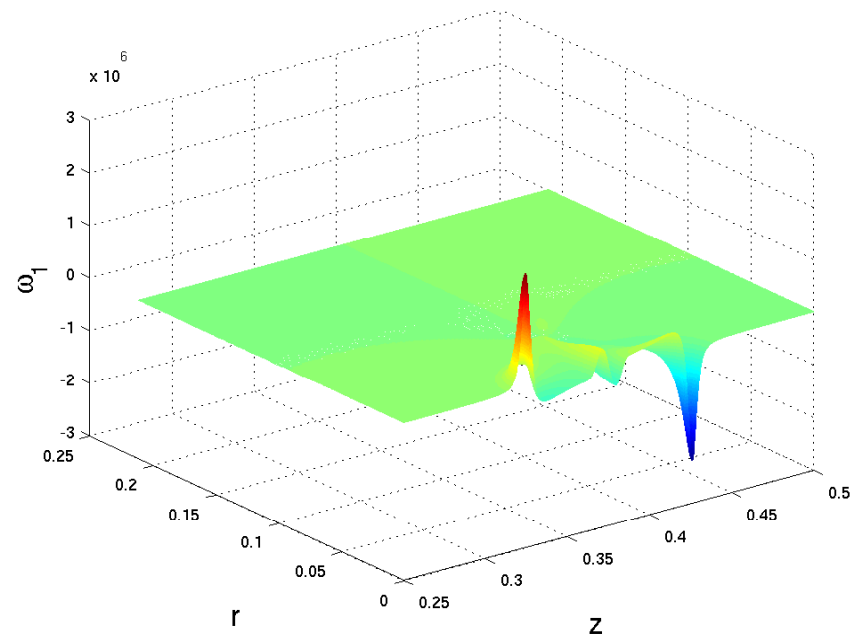

FIGURE 6.12. The three-dimensional view of $\omega_{1}$ at $t=0.02$ for the viscous model computed by the adaptive mesh with $N_{z}=4096, N_{r}=$ $400, \Delta t=2.5 \times 10^{-7}, v=0.001$.

Remark 6.1. While the numerical results presented in this subsection seem to support that the solution of the viscous model develops a potential finite time singularity, we caution that this evidence is not yet conclusive. Based on the balance between the nonlinear vortex-stretching term and the viscous term, we find that the solution of the viscous model seems to be dominated by the dynamics of the inviscid model during the time interval of our computation. In order to determine whether the three-dimensional model actually develops a finite time singularity, 
we need to compute much closer to the potential singularity time with resolutions much higher than what we have used in the current paper in order to capture the viscous effect accurately. Depending on the local scaling property of the nearly singular solution and the balance between the vortex-stretching term and the viscous term, it is still possible that the viscous term eventually regularizes the nearly singular solution induced by the nonlinear vortex-stretching term. We plan to investigate this issue further in our future work.

\section{Resolution Study}

Finally, we perform a resolution study for our computations by comparing the computation obtained by three different resolutions, which are $N_{z} \times N_{r}=2048 \times$ $256, N_{z} \times N_{r}=3072 \times 328$, and $N_{z} \times N_{r}=4096 \times 400$. In Figure 6.13, we plot $\left\|u_{1}\right\|_{\infty}$ as a function of time using these three resolutions, $N_{z} \times N_{r}=2048 \times 256$ (green), $N_{z} \times N_{r}=3072 \times 328$ (red), and $N_{z} \times N_{r}=4096 \times 400$ (blue) over the time interval [0,0.021]. We can see that while the computation with $N_{z}=2048$ underresolves the solution near the end of the computation, the solution obtained by using $N_{z}=3072$ gives an excellent agreement with that obtained by using $N_{z}=4096$.

We also compare the solution of $u_{1}$ at $r=0$ using three different resolutions. Using the partial regularity theory for our three-dimensional model, which we prove in [16], any singularity of our three-dimensional model must lie on the symmetry axis, $r=0$. Thus it makes sense to perform a resolution study for the solution along the symmetry axis that is the most singular region of the solution.

In the top box of Figure 6.14, we plot the solutions obtained by two resolutions using $N_{z} \times N_{r}=2048 \times 256\left(\Delta t=5 \times 10^{-7}\right)$ and $N_{z} \times N_{r}=4096 \times 400$ $\left(\Delta t=2.5 \times 10^{-7}\right)$ on top of each other at $t=0.02$. The two solutions are almost indistinguishable. However, the computation with $N_{z} \times N_{r}=2048 \times 256$ is not sufficient to resolve the nearly singular behavior of the solution at $t=0.021$. On the other hand, the computation with $N_{z} \times N_{r}=3072 \times 328(\Delta t=3.625 \times$ $10^{-7}$ ) gives much improved resolution. In Figure 6.14 (right plot), we compare the solution obtained by using $N_{z} \times N_{r}=3072 \times 328$ with that obtained by using $N_{z} \times N_{r}=4096 \times 400$ at $t=0.021$. We observe that the agreement of the two solutions is very good except near the points where $u_{1}$ attains its maximum.

\subsection{Singularity Formation for the Inviscid Model}

In this subsection, we present numerical evidence which seems to suggest that the corresponding inviscid model with the same initial condition develops a finite time singularity. The nature of the potential singularity will be studied, and the resolution study with effective resolution up to $8192^{3}$ will be performed.

\section{Numerical Evidence for a Potential Finite Time Singularity}

The solution's behavior of the inviscid model is qualitatively similar to that of the viscous model with viscosity coefficient $v=0.001$. At early times, the 

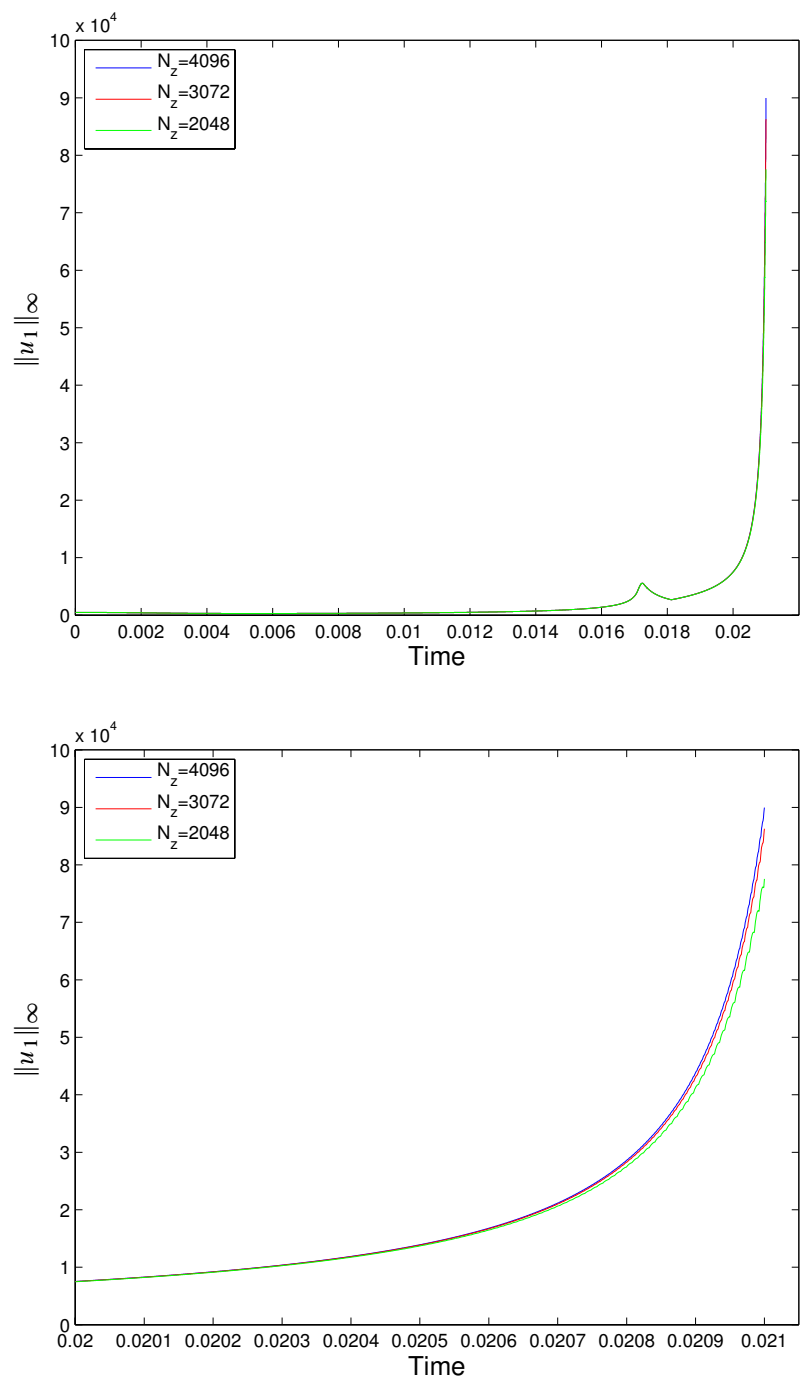

FIGURE 6.13. Convergence study for $\left\|u_{1}\right\|_{\infty}$ in time for the viscous model with three resolutions: $N_{z} \times N_{r}=2048 \times 256, \Delta t=5 \times 10^{-7}$ (green); $N_{z} \times N_{r}=3072 \times 328, \Delta t=3.625 \times 10^{-7}$ (red); $N_{z} \times N_{r}=$ $4096 \times 400, \Delta t=2.5 \times 10^{-7}$ (blue). The top figure is over the time interval $[0,0.021]$, while the bottom figure is a closeup view over the time interval $[0.02,0.021] . v=0.001$.

solution forms two large focusing centers that approach each other. Again these two focusing centers are strongly squeezed and form a thin layer parallel to the $r$-axis. We will show contour plots for $u_{1}$ very close to the predicted blowup 

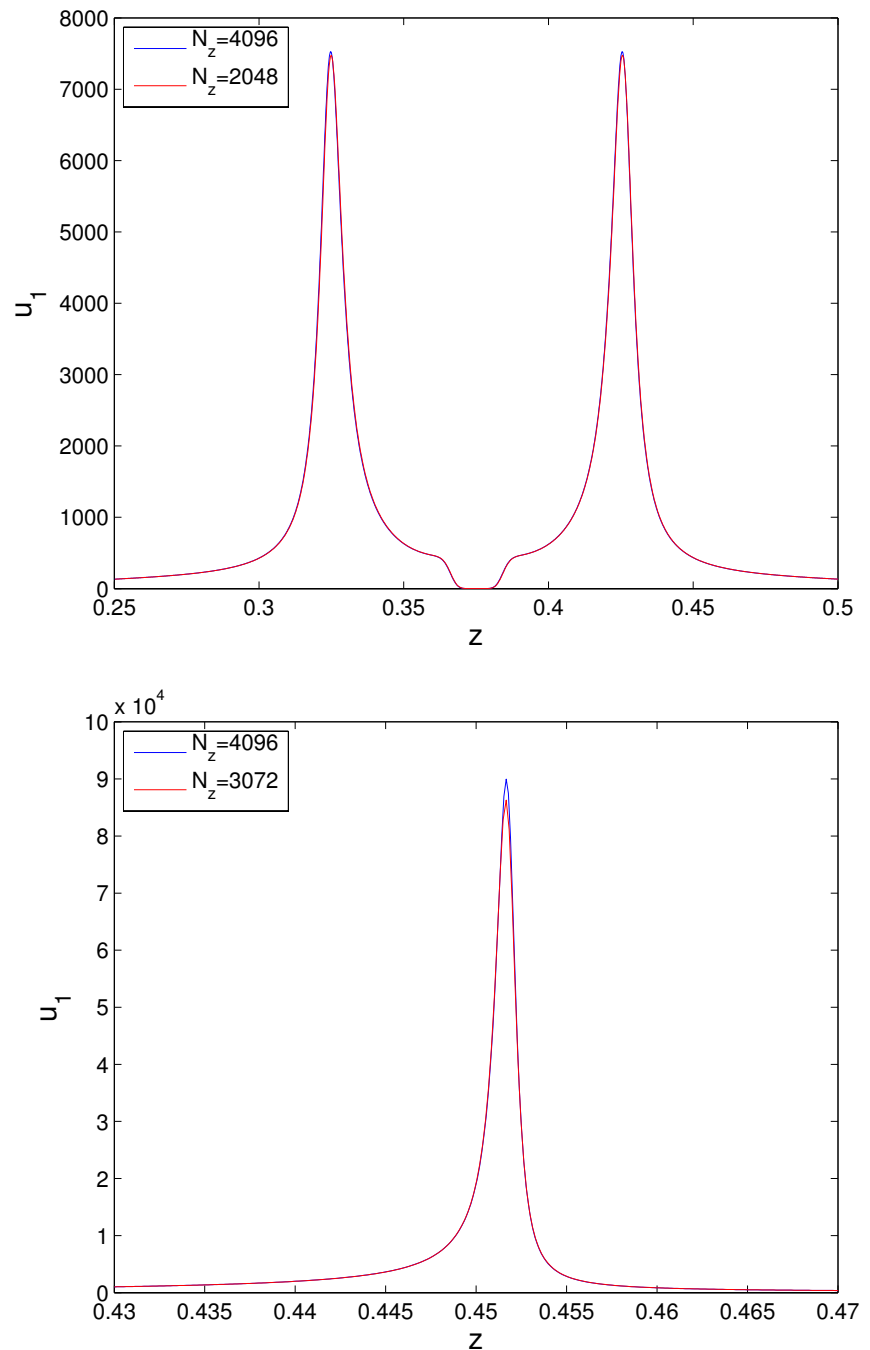

FIgURE 6.14. Convergence study for $u_{1}$ at $r=0$ and $t=0.02$ and $t=0.021$ for the viscous model with different resolutions. The top figure is the comparison between $N_{z} \times N_{r}=2048 \times 256$ (blue) and $N_{z} \times N_{r}=4096 \times 400$ (red). The bottom figure is the comparison between $N_{z} \times N_{r}=3072 \times 328$ (red) and $N_{z} \times N_{r}=4096 \times 400$ (blue). $v=0.001$.

time. In Figure 6.15, we plot the contours of $u_{1}$ and $\left(\psi_{1}\right)_{z}$ at $t=0.022$. The solution already develops a nearly singular behavior at this time. This becomes more evident by the time $t=0.0223$; see Figure 6.16. This suggests that the solution may eventually develop a finite time singularity. 

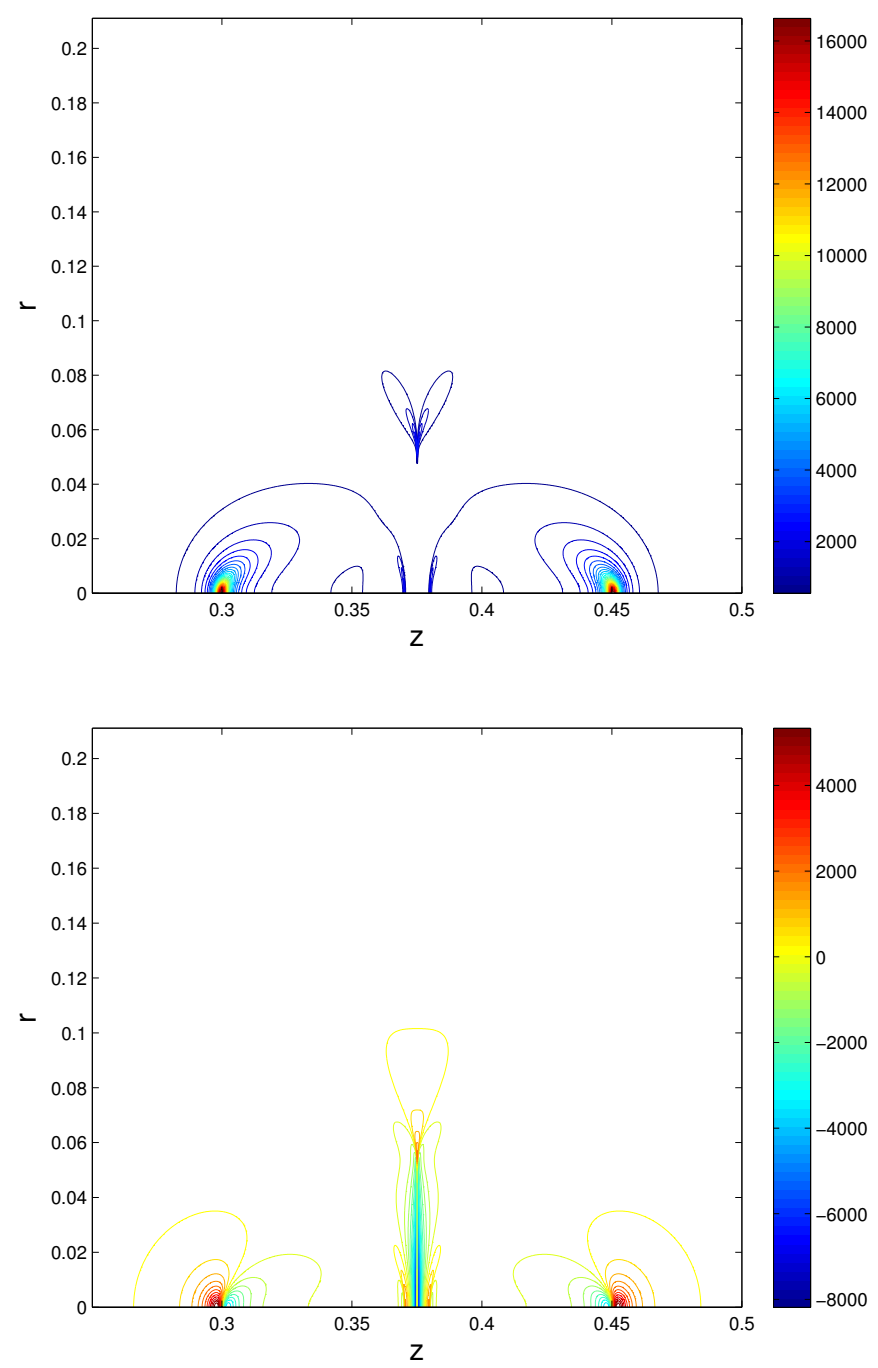

FIGURE 6.15. Contour plots of $u_{1}$ (top figure) and contours of $\left(\psi_{1}\right)_{z}$ for the inviscid model at $t=0.022$ (bottom figure) computed by the adaptive mesh $\left(N_{z}=4096, N_{r}=400\right), v=0$.

Next, we present further numerical evidence that seems to support the formation of a finite time singularity. We apply the same form fit procedure described earlier for the viscous model to the solution of the inviscid model. Our numerical study indicates that the singularity scaling of the inviscid model is slightly different from that of the viscous model. In particular, there seems to be a logarithmic correction 

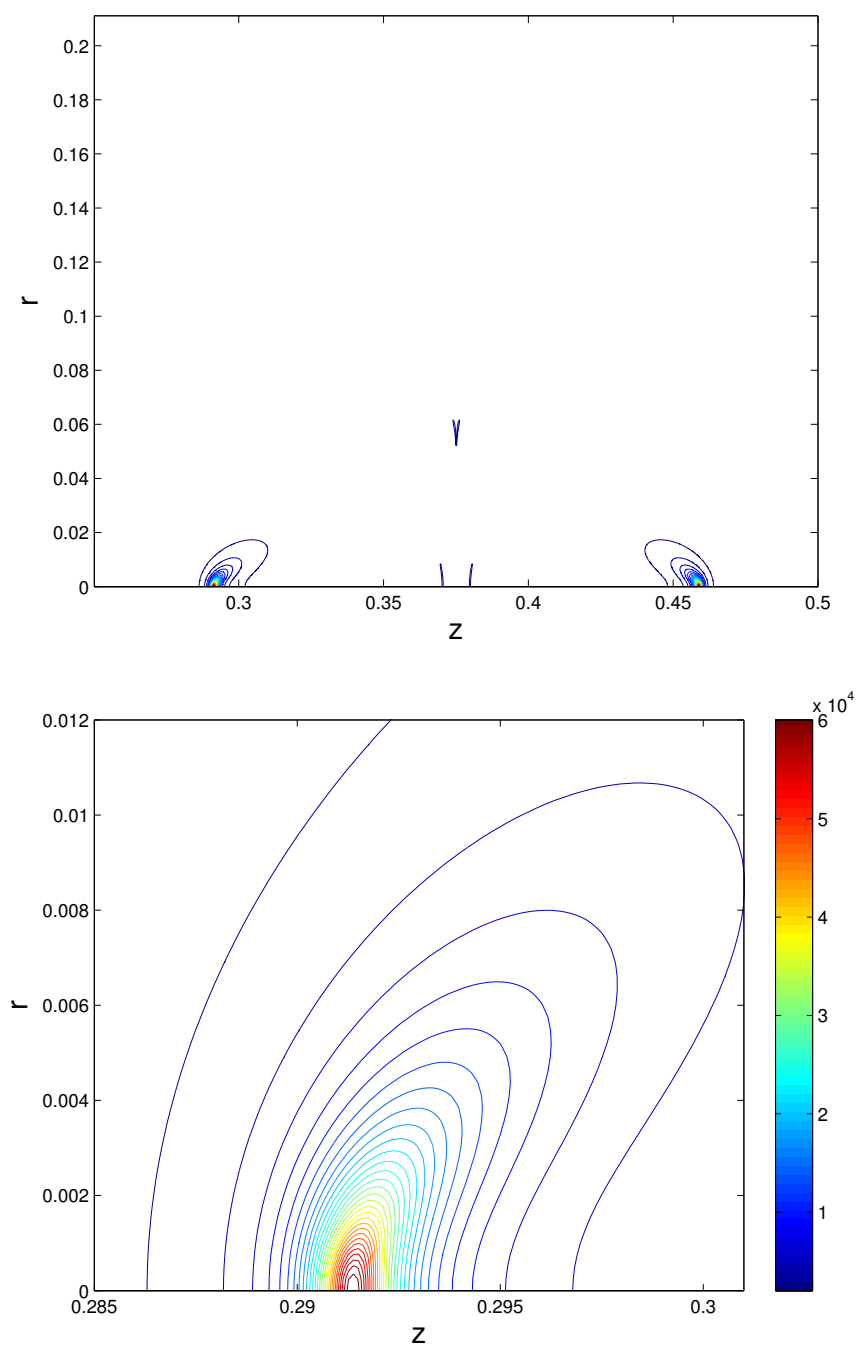

FIGURE 6.16. The contours of $u_{1}$ at $t=0.0223$ (top figure) and its closeup view (bottom figure) for the inviscid model computed by the adaptive mesh $\left(N_{z}=4096, N_{r}=400\right), v=0$.

in the potential blowup rate of $\left\|u_{1}\right\|_{\infty}$. We look for a finite time singularity of the form

$$
\left\|u_{1}\right\|_{\infty} \approx \frac{C}{(T-t)^{\alpha}(\log (1 /(T-t)))^{\beta}} .
$$

In Figure 6.17, we present numerical evidence which seems to imply that the solution of the inviscid model may develop a potential finite time singularity. With 


\begin{tabular}{|c|l|l|}
\hline$h_{z}$ & \multicolumn{1}{|c|}{$T$} & \multicolumn{1}{c|}{$C$} \\
\hline $1 / 4096$ & 0.02241 & 19.50302 \\
\hline $1 / 8192$ & 0.022404 & 19.46807 \\
\hline $1 / 16384$ & 0.02240 & 19.41995 \\
\hline Extrapolation to $h_{z}=0$ & 0.022395 & 19.351399 \\
\hline
\end{tabular}

TABLE 6.2. Resolution study of parameters $T$ and $C$ in the asymptotic fit for the inviscid model

$$
\left\|u_{1}\right\|_{\infty}^{-1} \approx \frac{(T-t)(\log (1 /(T-t)))^{1 / 2}}{C}
$$

using different resolutions $h_{z}=1 /\left(2 N_{z}\right), v=0$. The resolutions we use in our adaptive computations are $N_{z}=2048,4096$, and 8192. The corresponding time steps are $\Delta t=5 \times 10^{-7}, 2.5 \times 10^{-7}$, and $1.25 \times 10^{-7}$, respectively. The last row is obtained by extrapolating the second-order polynomial that interpolates the three data points.

resolution $N_{z} \times N_{r}=4096 \times 400$ and $\Delta t=2.5 \times 10^{-7}$, we find that the predicted singularity time is at $T=0.022404$, and the singularity exponent is $\alpha=1$ with a logarithmic correction $\left(\beta=\frac{1}{2}\right)$. The agreement between the computed solution and the form fit solution $C /\left((T-t)(\log (1 /(T-t)))^{1 / 2}\right)$ is almost indistinguishable in the final stage of the computation. We remark that the asymptotic blowup rate $C /\left((T-t)(\log (1 /(T-t)))^{1 / 2}\right)$ is consistent with the nonblowup criterion of BealeKato-Majda type; see Section 7.

To provide further evidence of a potential finite time singularity, we use a sequence of resolutions to study the limiting behavior of the computed solution as we refine our resolutions. The space resolutions we use are $N_{z} \times N_{r}=2048 \times 256$, $4096 \times 400$, and $8192 \times 800$. The corresponding time steps are $\Delta t=5 \times 10^{-7}$, $2.5 \times 10^{-7}$, and $1.25 \times 10^{-7}$, respectively. We obtain an optimal least squares singularity fit of the form $\left\|u_{1}\right\|_{\infty} \approx C /\left((T-t)(\log (1 /(T-t)))^{1 / 2}\right)$ for each resolution. The results are summarized in Table 6.2. Based on the fitted parameters $T$ and $C$ from these three resolutions, we construct a second-order polynomial that interpolates $T$ and $C$ through these three data points. We then use the polynomial to extrapolate the values of $T$ and $C$ to the infinite resolution limit. The extrapolated values at $h_{z}=0$ are $T=0.022395$ and $C=19.351399$.

In Figure 6.18, we plot the inverse of $\left\|u_{1}\right\|_{\infty}$ as a function of time using three different resolutions. We can see that as we refine the resolution, the computed solution converges to the extrapolated singularity limiting profile. This seems to support that the solution of the inviscid model may develop a potential finite time singularity.

\section{Resolution Study}

Now we perform a careful resolution study for our computations. We first study the convergence between the resolution $N_{z} \times N_{r}=2048 \times 256$ and the resolution 

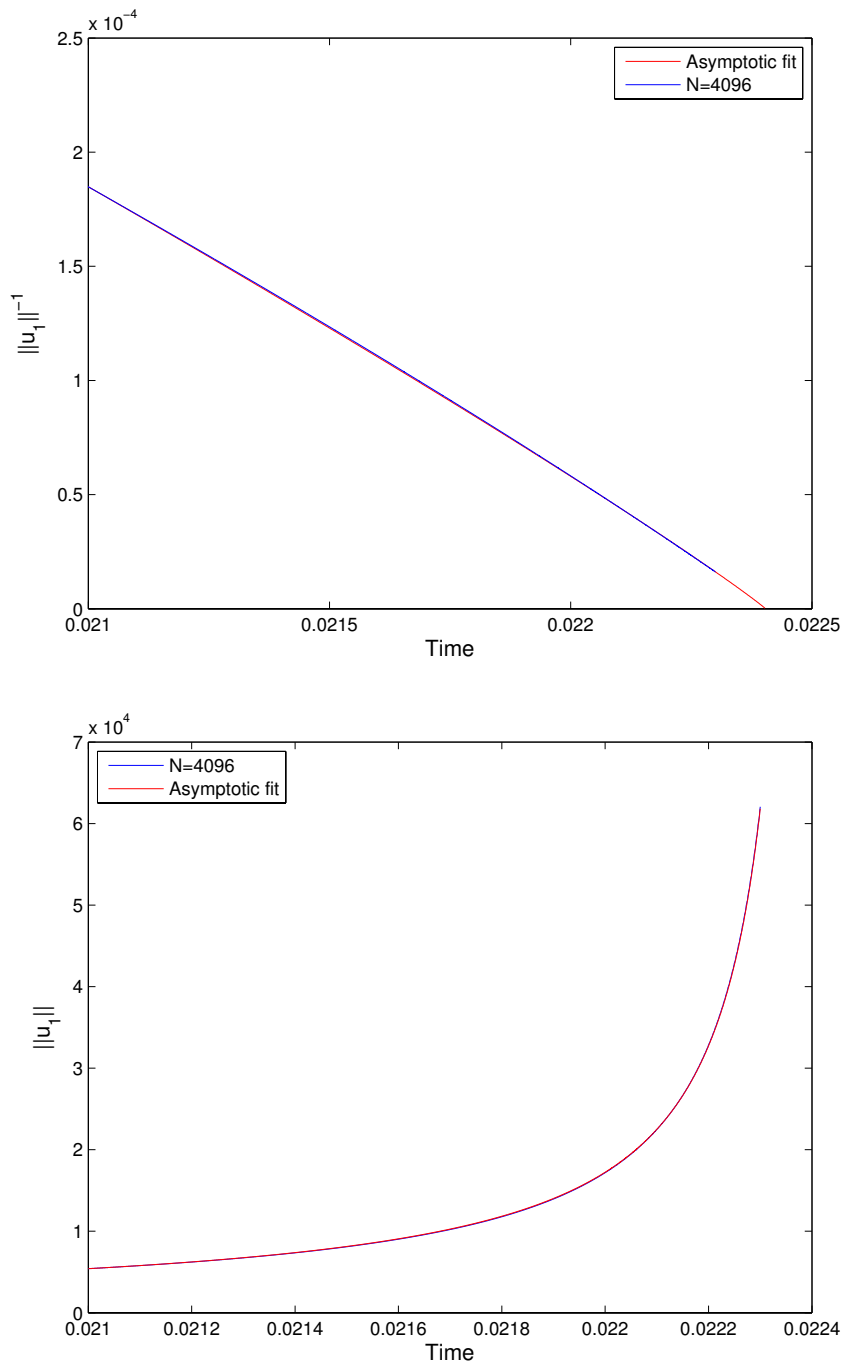

FigURE 6.17. Top plot: The inverse of $\left\|u_{1}\right\|_{\infty}$ (blue) versus the singularity form fit (red) for the inviscid model. Bottom plot: $\left\|u_{1}\right\|_{\infty}$ (blue) versus the singularity form fit (red). The singularity form fit is of the form

$$
\left\|u_{1}\right\|_{\infty} \approx \frac{C}{(T-t)(\log (1 /(T-t)))^{1 / 2}}
$$

with $T=0.022404$ and $C=19.46807$. The solution is computed by the adaptive mesh with $N_{z}=4096, N_{r}=400, \Delta t=2.5 \times 10^{-7}, v=0$. 


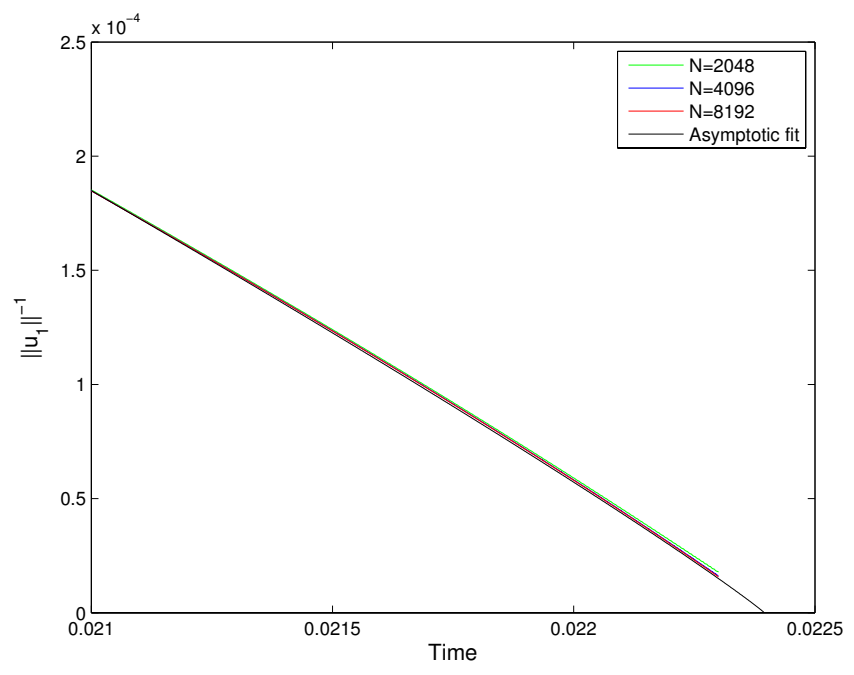

FIGURE 6.18. The inverse of $\left\|u_{1}\right\|_{\infty}$ in time for the inviscid model. The solution is computed by adaptive mesh with $N_{z}=2048,4096$, and 8192 (ordering from top to bottom in the figure), $\Delta t=5 \times 10^{-7}, 2.5 \times 10^{-7}$, and $1.25 \times 10^{-7}$, respectively. The last curve is the singularity fit by extrapolating the computational results to infinite resolution $N_{z}=\infty$. The fitted curve is of the form

$$
\left\|u_{1}\right\|_{\infty}^{-1} \approx \frac{(T-t)(\log (1 /(T-t)))^{1 / 2}}{C},
$$

with $T=0.0223953$ and $C=19.351399, v=0$.

$N_{z} \times N_{r}=4096 \times 400$. In Figure 6.19 (top plot), we show the maximum of $u_{1}$ in time up to $t=0.0223$. We find that the agreement is quite good. However, the $N_{z} \times N_{r}=2048 \times 256$ resolution seems to underresolve the solution toward the end of the computation. To make sure that $N_{z} \times N_{r}=4096 \times 400$ is sufficient to resolve the nearly singular solution behavior, we perform our largest computation using $N_{z} \times N_{r}=8192 \times 800$. To save computational time, we start this computation at $t=0.02$ using the numerical solution computed by $N_{z} \times N_{r}=4096 \times 400$ as the initial data. We realize that restarting the computation with $N_{z}=8192$ using the solution at $t=0.02$ obtained with $N_{z}=4096$ is less accurate than if it had started at $t=0$. On the other hand, due to our current limited computational resources, it would take more than two and a half months to complete the computation with $N_{z}=8192$ that is started at $t=0$. In Figure 6.19 (bottom plot), we compare $\left\|u_{1}\right\|_{\infty}$ computed by $N_{z} \times N_{r}=4096 \times 400$ with that computed by $N_{z} \times N_{r}=$ $8192 \times 800$. We can see that there is almost no difference between the two solutions. We also compare the solution of $u_{1}$ at $r=0$ using the two largest resolutions in Figure 6.20 (bottom plot). We plot the solutions obtained by the two resolutions 

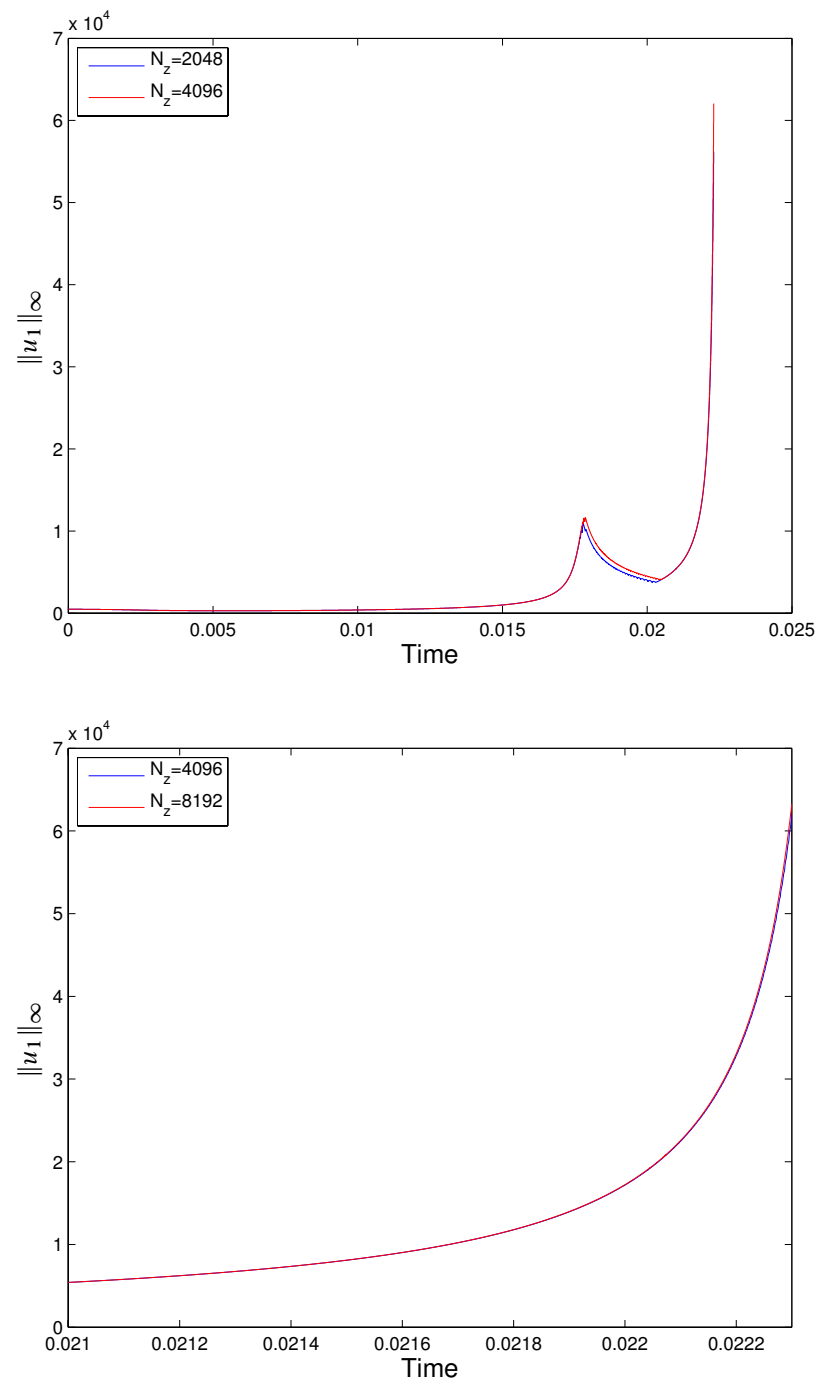

FIGURE 6.19. Convergence study for $\left\|u_{1}\right\|_{\infty}$ for the inviscid model with two resolutions up to $t=0.0223$. The top figure is a convergence study of $\left\|u_{1}\right\|_{\infty}$ between the resolution $N_{z}=2048, N_{r}=256$, $\Delta t=5 \times 10^{-7}$ (blue) and the resolution $N_{z}=4096, N_{r}=400$, $\Delta t=2.5 \times 10^{-7}$ (red). The bottom figure is a convergence study of $\left\|u_{1}\right\|_{\infty}$ between the resolution $N_{z}=4096, N_{r}=400, \Delta t=2.5 \times 10^{-7}$ (blue) and the resolution $N_{z}=8192, N_{r}=800, \Delta t=1.25 \times 10^{-7}$ (red). $v=0$. 
on top of each other at $t=0.0223$. The agreement between the two solutions is excellent.

We remark that the numerical evidence for singularity formation of the inviscid model is not yet conclusive. In a subsequent paper, we will use a fully adaptive computational method on a parallel cluster to perform well-resolved computations with resolutions that are substantially higher than what we have used in this paper. This is necessary to determine whether the solution will indeed develop a finite time singularity.

\section{Local Scaling Property of the Potential Singularity}

In this subsection, we further examine the nature of the potential singularity. Our preliminary numerical study seems to suggest that the potential singularity is locally self-similar and isotropic. In order to study the local scaling property of the potential singularity, we look for a dynamically rescaled profile $U(\xi, \eta, t)$ near the region of a potential singularity point such that

$$
u_{1}(z, r, t)=\frac{C}{(T-t)} U\left(\frac{z-z_{0}(t)}{(T-t)^{\beta}}, \frac{r}{(T-t)^{\beta}}, t\right) \quad \text { as } t \rightarrow T,
$$

where $T$ and $C$ are the values in our singularity form fit

$$
\left\|u_{1}\right\|_{\infty}^{-1} \approx \frac{(T-t)(\log (1 /(T-t)))^{1 / 2}}{C}
$$

$\beta$ is a parameter to be determined, and $z_{0}(t)$ is the location in which $\left|u_{1}\right|$ achieves its global maximum at $t$.

We investigate this local self-similarity property of the solution of the inviscid model in Figure 6.21. We use the computed solution at $t=0.022$ and 0.0223 with $N_{z} \times N_{r}=8192 \times 800\left(\Delta t=1.25 \times 10^{-7}\right)$ to determine the exponent $\beta$. In this case, we have $T=0.02240$ and $C=19.41995$ from Table 6.2. A least squares fit gives $\beta=\frac{3}{4}$. In Figure 6.21 (top plot), we show $U$ as a function of $\xi$ along the symmetry axis $\eta=0$. In the same figure (bottom plot), we show $U$ as a function of $\eta$ along the cross section $\xi=0$. As we can see, the dynamically rescaled profile $U$ as a function of the rescaled variables $\xi$ and $\eta$ seems to converge to a well-defined profile.

We also plot the growth rate of $\left\|\psi_{1}\right\|_{\infty}$ in Figure 6.22. In the top figure, we plot the inverse of $\left\|\psi_{1}\right\|_{\infty}$ as a function of time. We find that the computed solution agrees well with the fitted decay rate, $(T-t)^{1 / 4} /(C \log (1 /(T-t)))$. In the bottom plot, we compare $\left\|\psi_{1}\right\|_{\infty}$ with the asymptotic fit and observe a good agreement.

The preliminary results we presented above seem to suggest the following local scaling property of $u_{1}, \omega_{1}$, and $\psi_{1}$ near the region of the potential singularity:

$$
\begin{gathered}
u_{1}(z, r, t)=\frac{U(\xi, \eta, t)}{(T-t)}, \quad \omega_{1}(z, r, t)=\frac{W(\xi, \eta, t)}{(T-t)^{7 / 4}}, \\
\psi_{1}(z, r, t)=\frac{\Psi(\xi, \eta, t)}{(T-t)^{1 / 4}},
\end{gathered}
$$



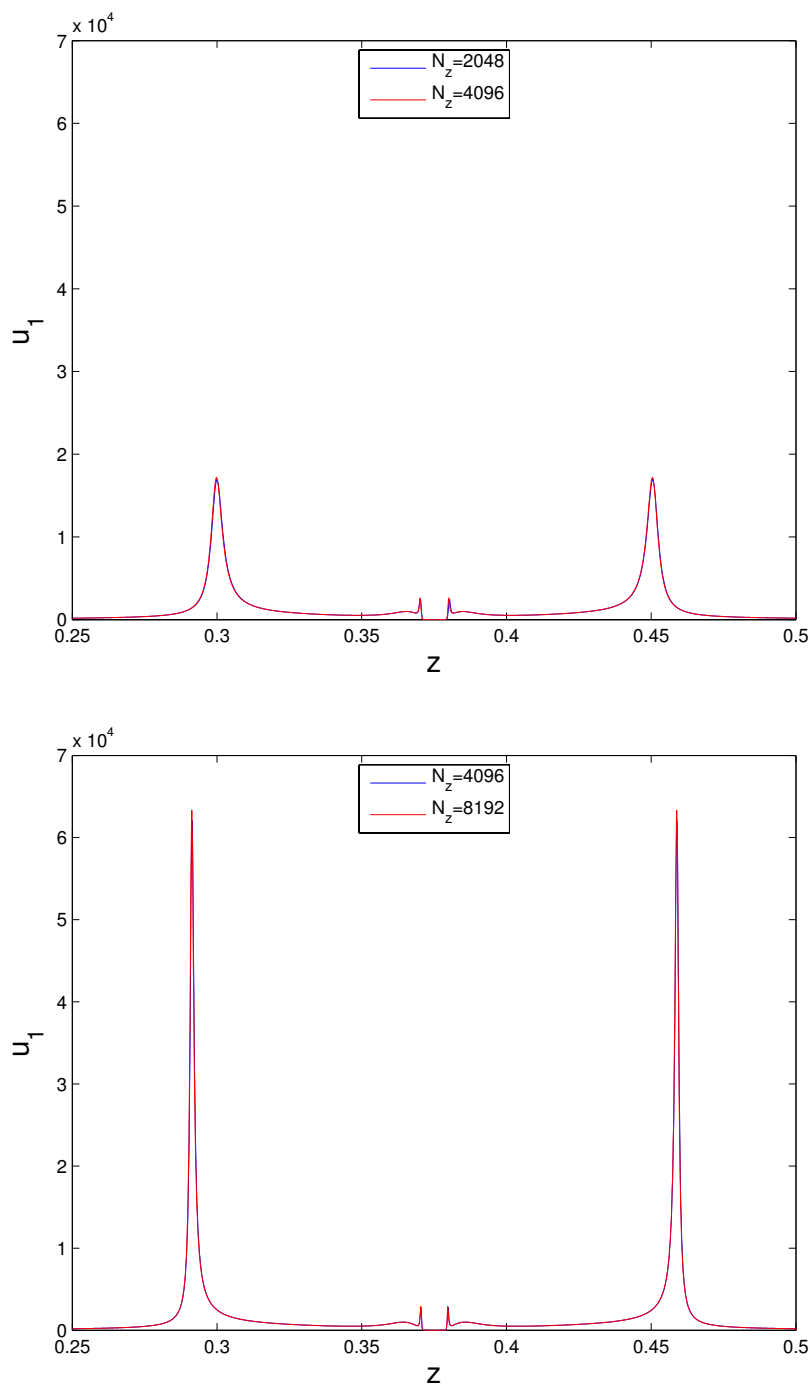

FIGURE 6.20. Convergence study of $u_{1}$ at $r=0$ for the inviscid model with different resolutions. The top figure is the comparison of $u_{1}$ at $t=0.022$ obtained by the resolution $N_{z}=2048, N_{r}=256$ (blue) and the resolution $N_{z}=4096, N_{r}=400$ (red), respectively. The bottom figure is the comparison of $u_{1}$ at $t=0.0223$ obtained by the resolution $N_{z}=4096, N_{r}=400$ (blue) and the resolution $N_{z}=8192, N_{r}=800$ (red), respectively. $v=0$. 

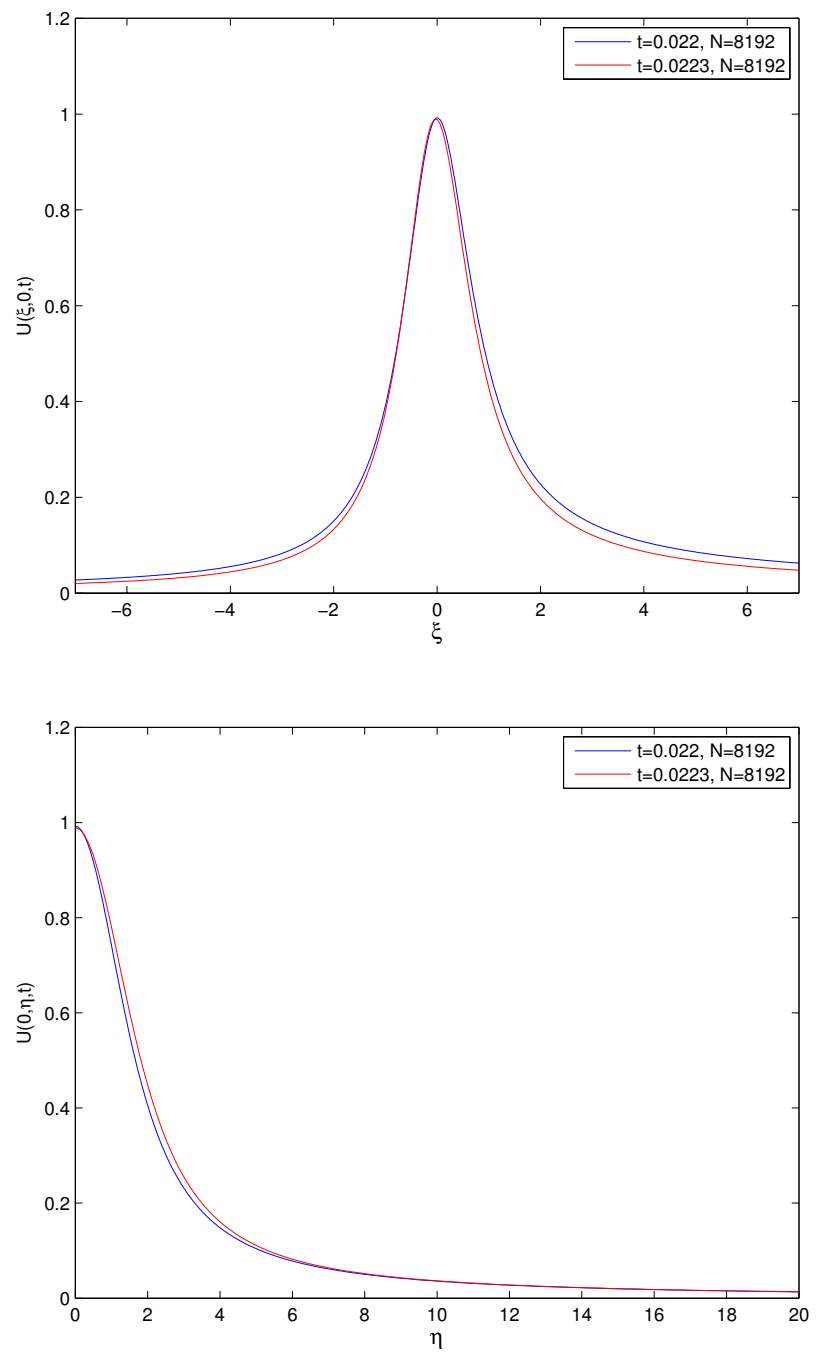

FIGURE 6.21. Rescaled profile for $u_{1}$ for the inviscid model at $t=$ 0.022 and 0.0223 , respectively. The dynamically rescaled profile $U(\xi, \eta, t)$ is defined through the relation

$$
u_{1}(z, r, t)=U(\xi, \eta, t) \frac{C}{(T-t)(\log (1 /(T-t)))^{1 / 2}},
$$

where $\xi=z-z_{0}(t) /(T-t)^{3 / 4}, \eta=r /(T-t)^{3 / 4}$, and $z_{0}(t)$ is the location where $\left|u_{1}\right|$ achieves its global maximum. Here $T$ and $C$ are the values in the singularity form fit given in Table $6.2\left(h_{z}=1 / 16,384\right)$. The top figure shows $U$ along the symmetry axis $\eta=0$, and the bottom figure shows $U$ along the cross section $\xi=0$. Adaptive mesh $N_{z}=8192$, $N_{r}=800, \Delta t=1.25 \times 10^{-7}, v=0$. 

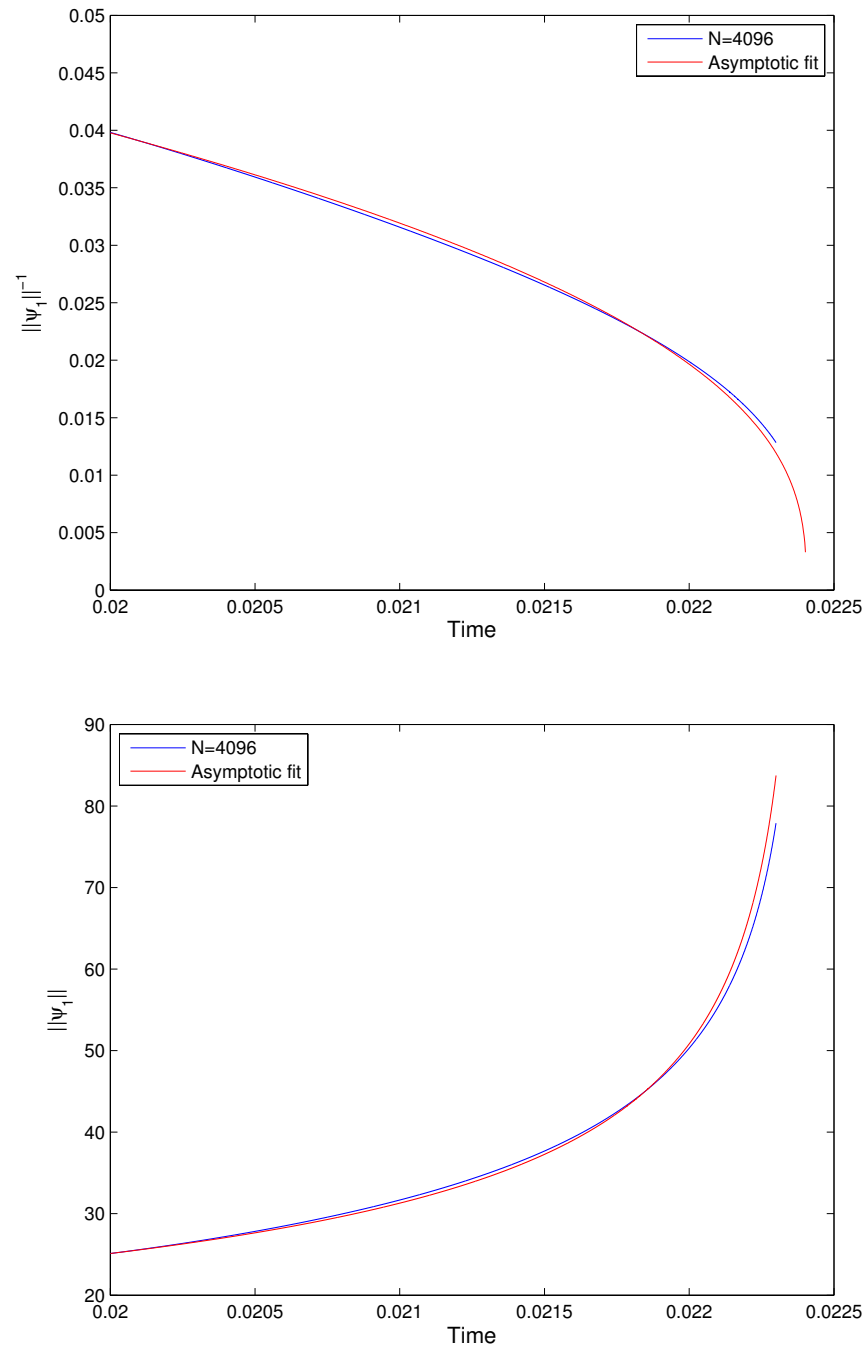

FIGURE 6.22. Top plot: The inverse of $\left\|\psi_{1}\right\|_{\infty}$ (blue) versus the asymptotic fit (red) for the inviscid model $(v=0)$. Bottom plot: $\left\|\psi_{1}\right\|_{\infty}$ (blue) versus the asymptotic fit (red). The asymptotic fit is of the form

$$
\left\|\psi_{1}\right\|_{\infty} \approx \frac{C \log (1 /(T-t))}{(T-t)^{1 / 4}}
$$

with $T=0.022404$ and $C=0.9221$. The solution is computed by adaptive mesh with $N_{z}=4096, N_{r}=400, \Delta t=2.5 \times 10^{-7}$. 
where

$$
\xi=\frac{z-z_{0}}{(T-t)^{3 / 4}}, \quad \eta=\frac{r}{(T-t)^{3 / 4}},
$$

and $z_{0}$ is the limiting value of $z_{0}(t)$ as $t \rightarrow T$. If we express the solution in terms of the velocity field $\left(u^{\theta}, u^{r}, u^{z}\right)$ defined in (3.2)-(3.3), we have

$$
u^{r}=\frac{U^{r}(\xi, \eta, t)}{(T-t)^{1 / 4}}, \quad u^{\theta}=\frac{U^{\theta}(\xi, \eta, t)}{(T-t)^{1 / 4}}, \quad u^{z}=\frac{U^{z}(\xi, \eta, t)}{(T-t)^{1 / 4}},
$$

where

$$
\xi=\frac{z-z_{0}}{(T-t)^{3 / 4}}, \quad \eta=\frac{r}{(T-t)^{3 / 4}},
$$

$U^{r}=-\eta(\Psi)_{\xi}, U^{\theta}=\eta U$, and $U^{z}=\left(\eta^{2} \Psi\right)_{\eta} / \eta$. The scaling property presented in (6.17) is consistent with the asymptotically self-similar scaling of the incompressible three-dimensional Euler equations.

We have also studied the local scaling property of the solution of the viscous model. Our preliminary study shows that the solution of the viscous model seems to have a locally self-similar profile under some appropriate rescaling. However, in order to determine the precise scaling exponent of the solution, we need to accurately capture the effect of the viscous term. For this purpose, we need to perform computation with a much higher resolution and solve the viscous model much closer to the potential singularity time. This is beyond the scope of the current paper and will be the subject of a future study.

\subsection{Mechanism for a Finite Time Blowup}

To understand the mechanism for the potential blowup of the viscous model, we plot the solution $u_{1}$ on top of $\left(\psi_{1}\right)_{z}$ along the symmetry axis $r=0$ at $t=0.021$ in Figure 6.23. We see that there is a significant overlap between the support of the maximum of $u_{1}$ and that of the maximum of $\left(\psi_{1}\right)_{z}$. Moreover, the solution $u_{1}$ has a strong alignment with $\left(\psi_{1}\right)_{z}$ near the region of the maximum of $u_{1}$. The local alignment between $u_{1}$ and $\left(\psi_{1}\right)_{z}$ induces a strong nonlinearity on the righthand side of the $u_{1}$ equation, which has the form $\left(\psi_{1}\right)_{z} u_{1}$. This strong alignment between $u_{1}$ and $\left(\psi_{1}\right)_{z}$ is the main mechanism for the potential finite time blowup of the three-dimensional model. Similar alignment between $u_{1}$ and $\left(\psi_{1}\right)_{z}$ near the region of maximum $u_{1}$ is observed for the inviscid model; see Figure 6.24.

As we see in the next subsection, the inclusion of the convection term forces the two focusing centers to travel toward each other. Moreover, the local alignment between $u_{1}$ and $\left(\psi_{1}\right)_{z}$ is destroyed. As a result, the solution becomes defocused and smoother along the symmetry axis.

\subsection{Stabilizing Effect of the Convection Term}

In this subsection, we will show that by adding back the convection term to the three-dimensional model, which recovers the original Navier-Stokes equations, the solution behaves completely differently. The mechanism for generating the 

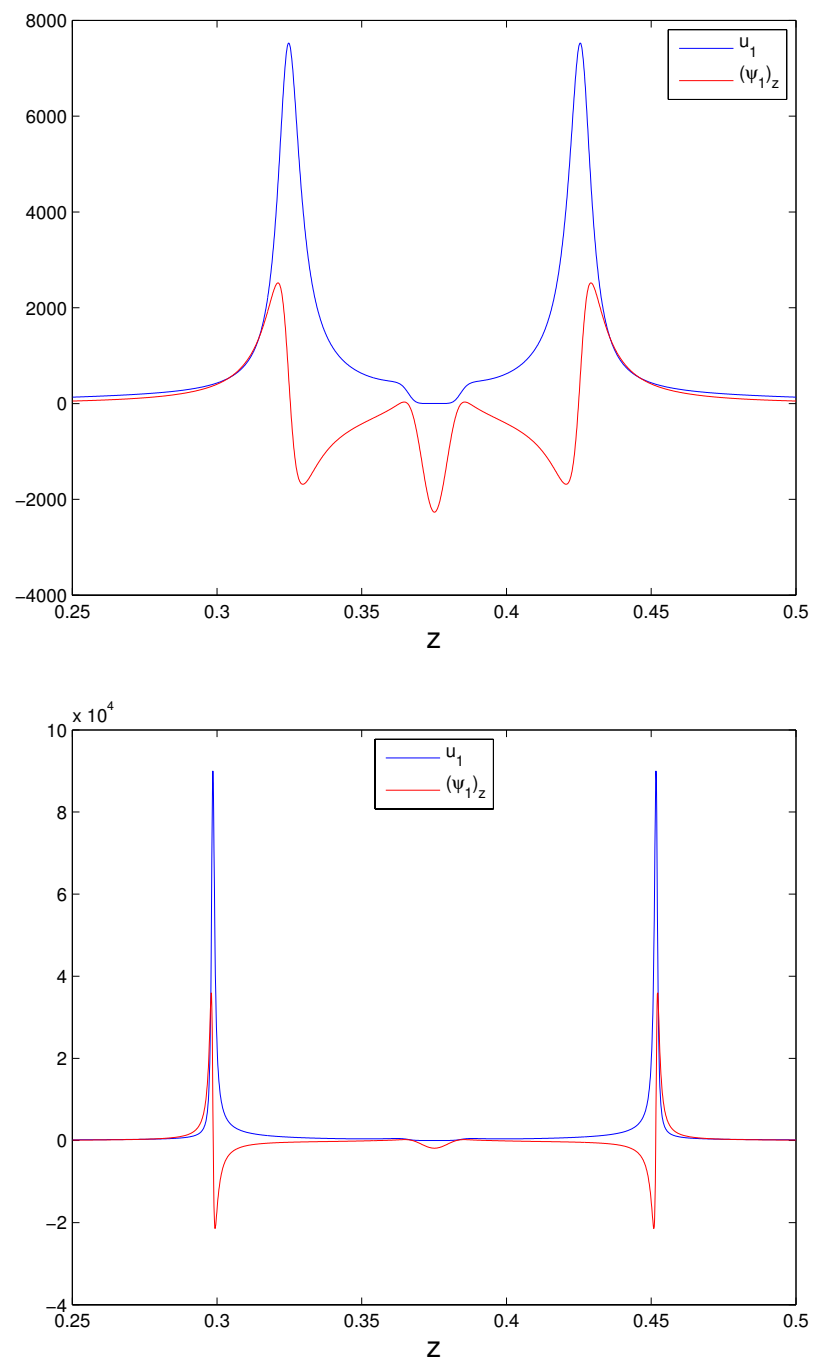

FIGURE 6.23. $u_{1}$ (blue) versus $\left(\psi_{1}\right)_{z}$ (red) of the viscous model along the symmetry axis $r=0$. The top figure corresponds to $t=0.02$. The bottom figure corresponds to $t=0.021$. Adaptive mesh computation with $N_{z}=4096, N_{r}=400, \Delta t=2.5 \times 10^{-7}, v=0.001$.

potential finite time singularity for the three-dimensional model is destroyed. Even if we start with the nearly singular solution obtained by the viscous model at $t=$ 0.02 and use it as the initial condition for the full Navier-Stokes equations, we observe that the maximum of $u_{1}$ soon decreases in time; see Figure 6.25. It is easy to see that the three-dimensional axisymmetric Navier-Stokes equations with swirl 

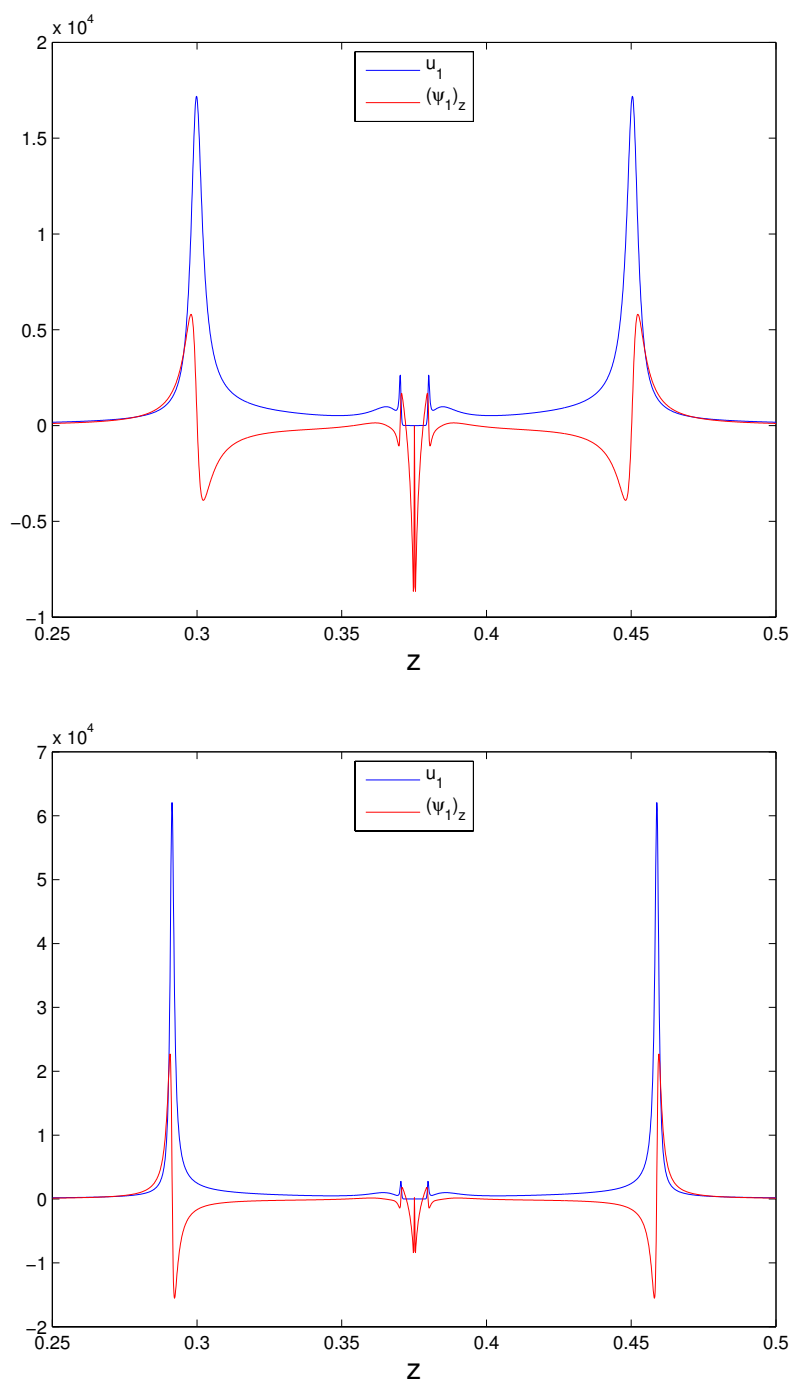

FIGURE 6.24. $u_{1}$ (blue) versus $\left(\psi_{1}\right)_{z}$ (red) of the three-dimensional inviscid model along the symmetry axis $r=0$. The top figure corresponds to $t=0.022$, and the bottom figure corresponds to $t=0.0223$. Adaptive mesh computation with $N_{z}=4096, N_{r}=400, \Delta t=2.5 \times 10^{-7}$, $v=0$.

cannot develop a finite time singularity if $u_{1}$ is bounded. Thus the fact that $\left\|u_{1}\right\|_{\infty}$ is decreasing in time is a clear indication that the solution does not develop a finite time singularity, at least over the time interval we consider here. 


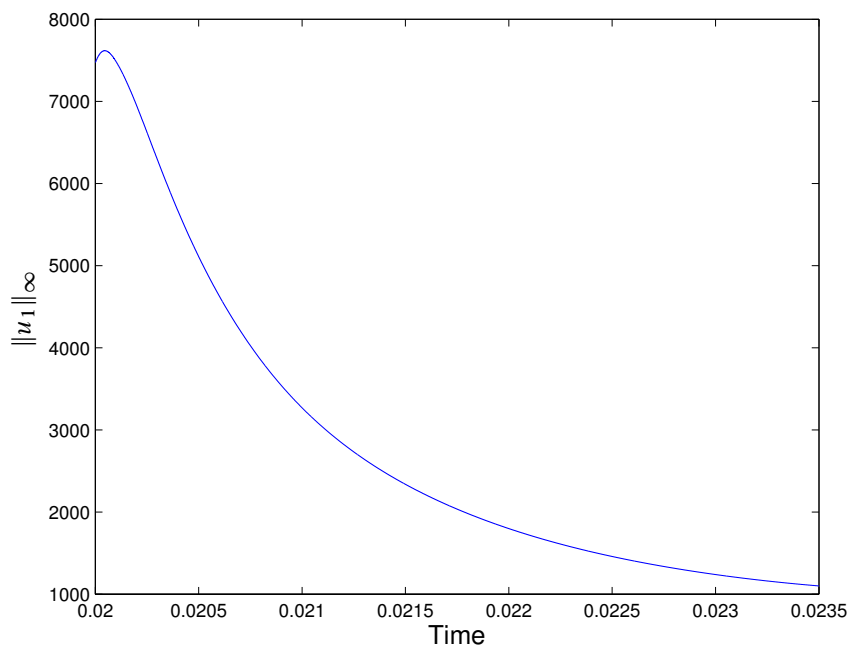

FIGURE 6.25. $\left\|u_{1}\right\|_{\infty}$ in time, the full Navier-Stokes computation using the solution of the three-dimensional viscous model at $t=0.02$ as the initial condition. Adaptive mesh computation with $N_{z}=2048, N_{r}=$ $1024, v=0.001$.

We also observe that the local alignment between $u_{1}$ and $\left(\psi_{1}\right)_{z}$ near the region of maximum $u_{1}$ is destroyed by including the convection term (see Figure 6.26), as is the focusing mechanism. The solution becomes defocused (see Figure 6.27). As time evolves, the two focusing centers approach each other. This process creates a strong internal layer orthogonal to the $z$-axis and forms a jet that moves away from the symmetry axis ( $z$-axis). The jet further generates some interesting vortex structures. This is illustrated in Figure 6.28. Since the solution of the full NavierStokes equations moves away from the symmetry axis, we use a higher-resolution adaptive mesh along the $r$-direction with $N_{r}=1024$ to better resolve the layered structure along the $r$-axis.

By the Caffarelli-Kohn-Nirenberg theory, the singularity of the three-dimensional axisymmetric Navier-Stokes equations, if there is any, must be along the symmetry axis. The fact that the most singular part of the solution moves away from the symmetry axis indicates that the full Navier-Stokes equations will not form a finite time singularity, at least not for the initial condition we consider here over the time interval for which we compute the solution. On the other hand, the solution of the three-dimensional model with the same initial condition seems to develop a potential finite time singularity in an earlier time. This confirms that convection plays an essential role in depleting the destabilizing effect induced by vortex stretching. 

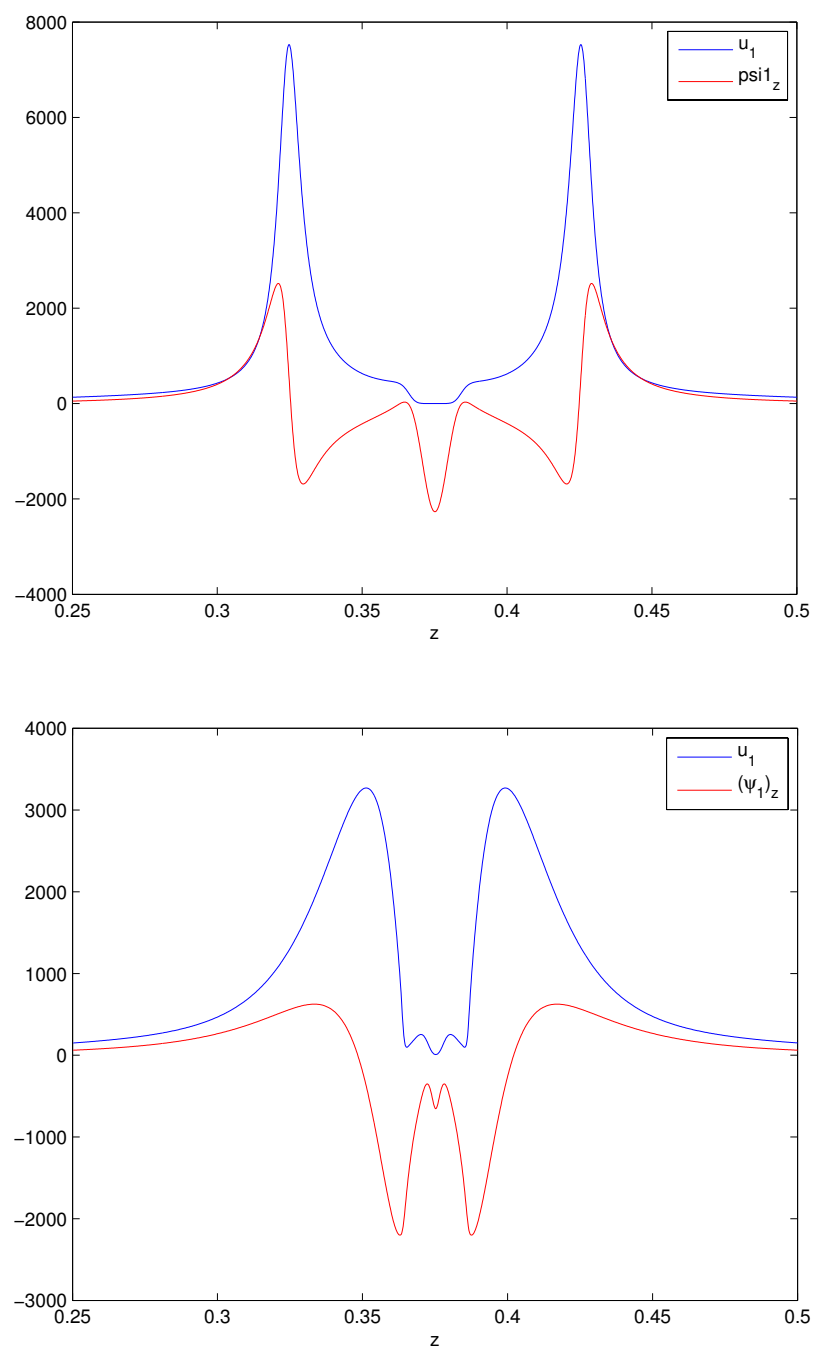

FIGURE 6.26. $u_{1}$ (blue) versus $\left(\psi_{1}\right)_{z}$ (red) along the symmetry axis $r=0$. The top figure corresponds to $t=0.02$ (the solution from the three-dimensional viscous model), and the bottom figure corresponds to $t=0.021$ obtained by solving the full Navier-Stokes equations. Adaptive mesh computation with $N_{z}=2048, N_{r}=1024, v=0.001$.

\section{A Nonblowup Criterion of Beale-Kato-Majda Type}

In this section, we prove a nonblowup criterion of the three-dimensional model equations (3.1) that is an analogue of the Beale-Kato-Majda (BKM) result for the three-dimensional Euler and Navier-Stokes equations. For the three-dimensional 

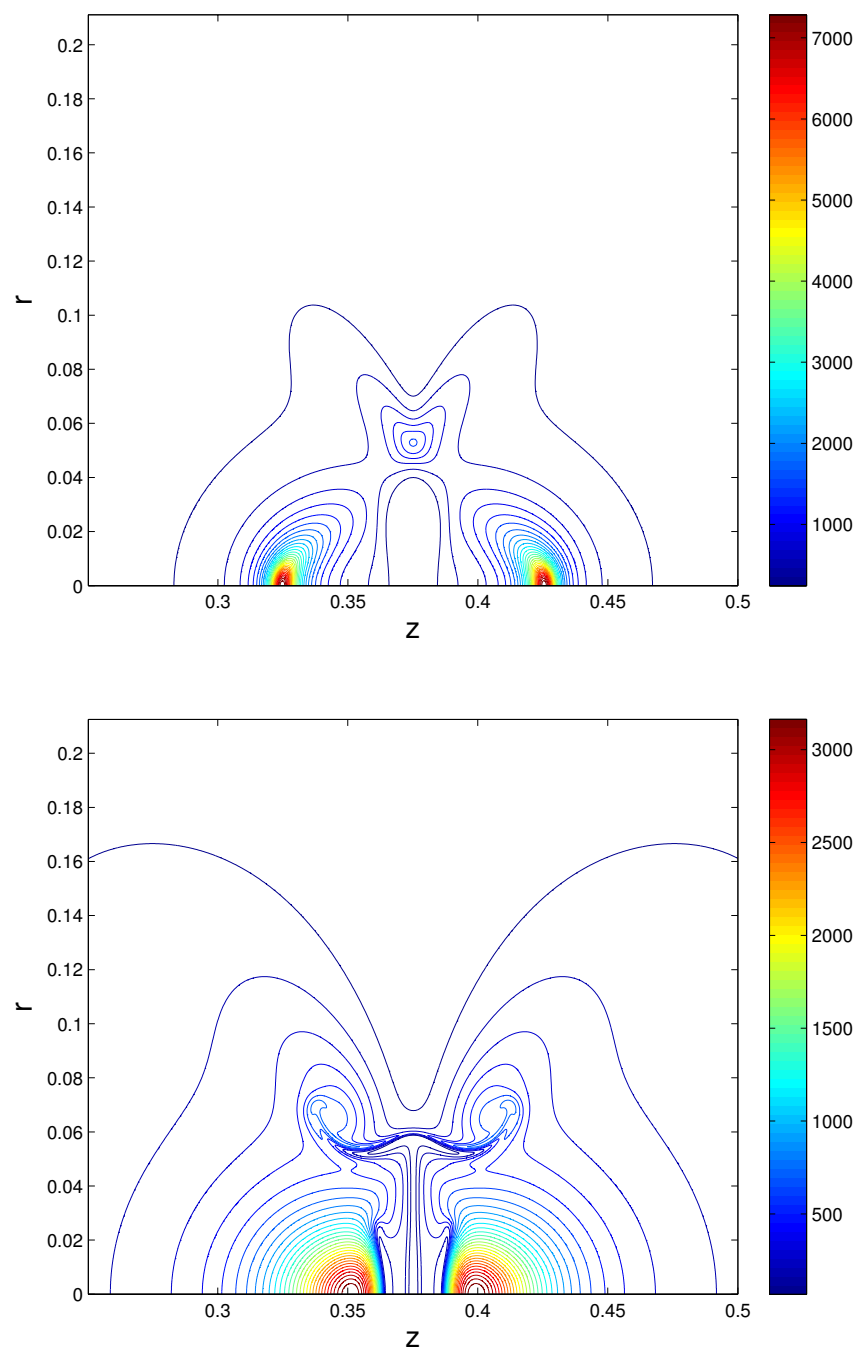

FIGURE 6.27. Top plot: The contour of $u_{1}$ at $t=0.02$ obtained from the three-dimensional viscous model that serves as the initial condition for the full Navier-Stokes equations. Bottom plot: The contour of $u_{1}$ at $t=0.021$ obtained by solving the full Navier-Stokes equations. Adaptive mesh computation with $N_{z}=2048, N_{r}=1024, v=0.001$.

Euler and Navier-Stokes equations, the BKM nonblowup criterion states that the solution $u$ blows up at time $T<\infty$ if and only if the accumulation of vorticity $\int_{0}^{T}\left\|\nabla_{x} \times u\right\|_{L^{\infty}\left(\mathbb{R}^{3}\right)} d t$ is infinite [1]. The BKM nonblowup criterion was later improved by Kozono and Taniuchi [22], who proved that the $L^{\infty}$ norm can be 

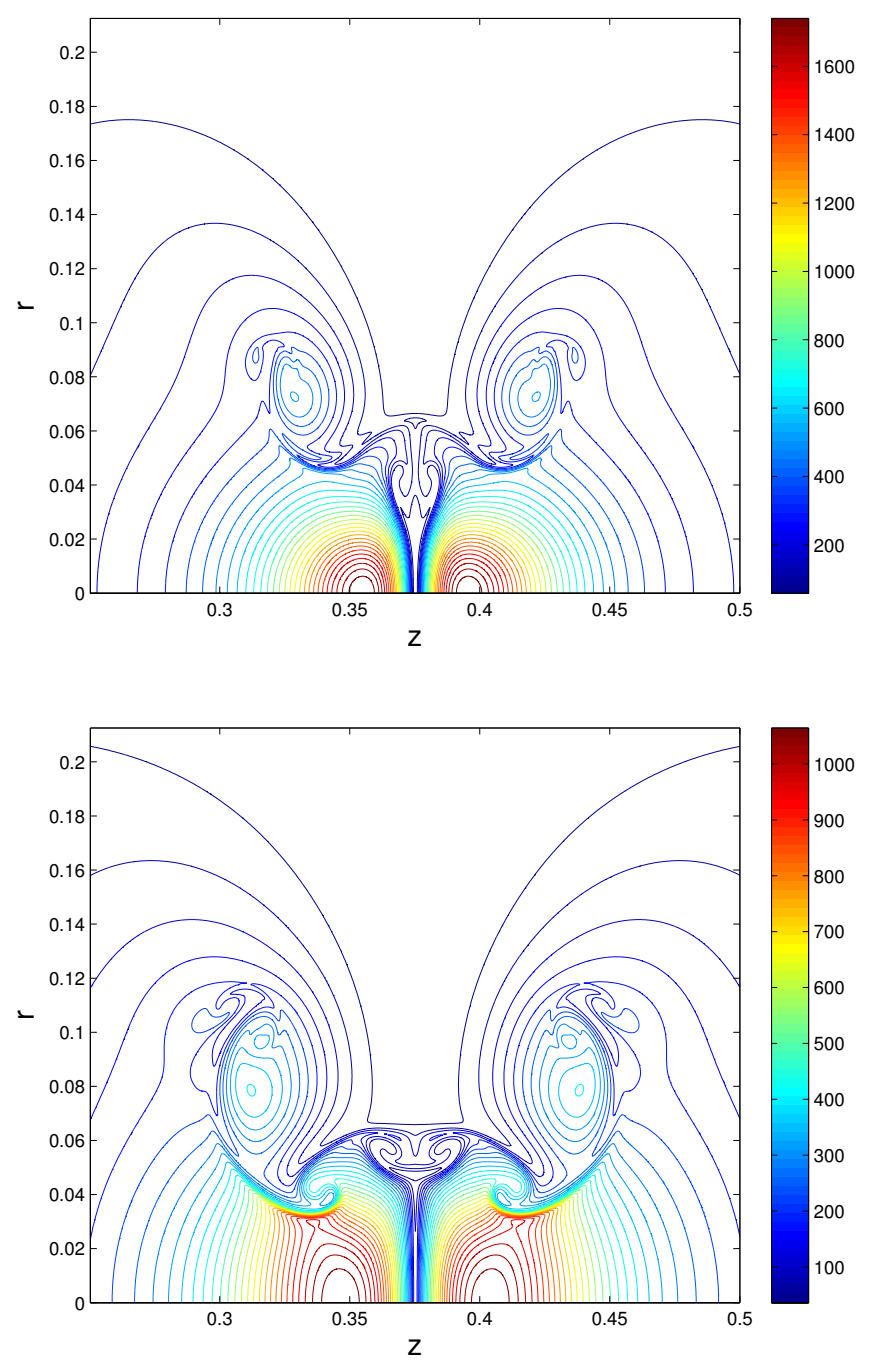

FIGURE 6.28. The contours of $u_{1}$ at $t=0.022$ (top plot) and $t=$ 0.0235 (bottom plot) by solving the full Navier-Stokes equations. Adaptive mesh computation with $N_{z}=2048, N_{r}=1024, v=0.001$.

replaced by the norm in the BMO space. This generalization is interesting because some crucial Sobolev embedding theorems can be applied to the BMO space, but not to the $L^{\infty}$ space. A nonblowup result formulated in terms of the BMO space has a broader range of applications.

THEOREM 7.1 Let $\left(u_{1}, \omega_{1}, \psi_{1}\right)$ be a solution of the three-dimensional model equations (3.1) that is regular for $0 \leq t<T$; that is, for all $t \in[0, T),\left(u_{1}, \nabla_{y} \psi_{1}\right) \in$ 
$H^{3}\left(\mathbb{R}^{5}\right)$. Assume that

$$
\int_{0}^{T}\left\|\nabla_{x} \times \mathbf{u}\right\|_{\mathrm{BMO}\left(\mathbb{R}^{3}\right)} d t<\infty
$$

then $\left(u_{1}, \omega_{1}, \psi_{1}\right)$ can be uniquely extended up to a time $T+\delta$ for some $\delta>0$ as a smooth solution of the three-dimensional model equations.

To prove the above theorem, we need the following lemma, which was proved by Kozono and Taniuchi in [22]:

LEMMA 7.2 Suppose that $\mathbf{u} \in H^{s}\left(\mathbb{R}^{3}\right)$ for some $s>\frac{5}{2}$ and

$$
\nabla_{x} \cdot \mathbf{u}=0, \quad \nabla_{x} \times \mathbf{u}=\omega .
$$

Then we have

$$
\left\|\nabla_{x} \mathbf{u}\right\|_{L^{\infty}\left(\mathbb{R}^{3}\right)} \leq C\left[1+\|\boldsymbol{\omega}\|_{\mathrm{BMO}\left(\mathbb{R}^{3}\right)} \ln \left(1+\|\mathbf{u}\|_{H^{s}\left(\mathbb{R}^{3}\right)}\right)\right] .
$$

We also need the following well-known lemma [26]:

LEMmA 7.3 Suppose that $f, g \in H^{s}\left(\mathbb{R}^{5}\right) \cap L^{\infty}\left(\mathbb{R}^{5}\right)$ for some $s \geq 0$. Then we have

$$
\left\|\nabla_{y}^{s}(f g)\right\|_{L^{2}\left(\mathbb{R}^{5}\right)} \leq C\left(\left\|\nabla_{y}^{s} f\right\|_{L^{2}\left(\mathbb{R}^{5}\right)}\|g\|_{L^{\infty}\left(\mathbb{R}^{5}\right)}+\left\|\nabla_{y}^{s} g\right\|_{L^{2}\left(\mathbb{R}^{5}\right)}\|f\|_{L^{\infty}\left(\mathbb{R}^{5}\right)}\right) .
$$

PROOF OF THEOREM 7.1: We only present the proof for the inviscid case. The proof of the viscous case follows similarly.

Applying the differential operator $\nabla_{y}^{3}$ to the first and second equations in (3.1) and then taking the $L^{2}$ inner product of the resulting equations with $\nabla_{y}^{3} u_{1}$ and $\nabla_{y}^{3} \psi_{1}$, we have

$$
\begin{aligned}
\frac{1}{2} & \frac{d}{d t}\left(\left\|\nabla_{y}^{3} u_{1}\right\|_{L^{2}\left(\mathbb{R}^{5}\right)}^{2}+\left\|\nabla_{y}^{4} \psi_{1}\right\|_{L^{2}\left(\mathbb{R}^{5}\right)}^{2}\right) \\
& =\int_{\mathbb{R}^{5}}\left[2 \nabla_{y}^{3}\left(\partial_{z} \psi_{1} u_{1}\right) \nabla_{y}^{3} u_{1}+\nabla_{y}^{3} \psi_{1} \nabla_{y}^{3} \partial_{z}\left(u_{1}^{2}\right)\right] d y \\
& =\int_{\mathbb{R}^{5}}\left[2 \nabla_{y}^{3}\left(\partial_{z} \psi_{1} u_{1}\right) \nabla_{y}^{3} u_{1}-\nabla_{y}^{3} \partial_{z} \psi_{1} \nabla_{y}^{3}\left(u_{1}^{2}\right)\right] d y .
\end{aligned}
$$

Using Lemma 7.3, we have

$$
\begin{aligned}
& \frac{1}{2} \frac{d}{d t}\left(\left\|\nabla_{y}^{3} u_{1}\right\|_{L^{2}\left(\mathbb{R}^{5}\right)}^{2}+\left\|\nabla_{y}^{4} \psi_{1}\right\|_{L^{2}\left(\mathbb{R}^{5}\right)}^{2}\right) \leq \\
& \quad C\left(\left\|u_{1}\right\|_{L^{\infty}\left(\mathbb{R}^{5}\right)}+\left\|\partial_{z} \psi_{1}\right\|_{L^{\infty}\left(\mathbb{R}^{5}\right)}\right)\left(\left\|u_{1}\right\|_{H^{3}\left(\mathbb{R}^{5}\right)}^{2}+\left\|\nabla_{y} \psi_{1}\right\|_{H^{3}\left(\mathbb{R}^{5}\right)}^{2}\right)
\end{aligned}
$$


By (3.17), it is easy to see that

$$
\begin{aligned}
\left|\nabla_{x} \mathbf{u}\right|^{2}= & \left(\left|\partial_{r} u^{\theta}\right|^{2}+\left|\partial_{z} u^{\theta}\right|^{2}+\left|\frac{u^{\theta}}{r}\right|^{2}\right)+\left[\partial_{r}^{2} \psi^{\theta}+\partial_{r}\left(\frac{\psi^{\theta}}{r}\right)\right]^{2} \\
& +\left|\partial_{z}^{2} \psi^{\theta}\right|^{2}+\left|\partial_{r z}^{2} \psi^{\theta}\right|^{2}+\left[\partial_{z}\left(\frac{\psi^{\theta}}{r}\right)\right]^{2} \\
& +\left[\partial_{z}\left(\frac{\psi^{\theta}}{r}\right)+\partial_{r z}^{2} \psi^{\theta}\right]^{2} \\
\geq & \left(\left|u_{1}\right|^{2}+\left|\partial_{z} \psi_{1}\right|^{2}\right) \geq \frac{1}{2}\left(\left|u_{1}\right|+\left|\partial_{z} \psi_{1}\right|\right)^{2} .
\end{aligned}
$$

Combining the energy identity (3.9) with (7.3)-(7.4), we arrive at

$$
\begin{aligned}
& \frac{d}{d t}\left(\left\|u_{1}\right\|_{H^{3}\left(\mathbb{R}^{5}\right)}^{2}+\left\|\nabla_{y} \psi_{1}\right\|_{H^{3}\left(\mathbb{R}^{5}\right)}^{2}\right) \leq \\
& C\left\|\nabla_{x} \mathbf{u}\right\|_{L^{\infty}\left(\mathbb{R}^{3}\right)}\left(\left\|u_{1}\right\|_{H^{3}\left(\mathbb{R}^{5}\right)}^{2}+\left\|\nabla_{y} \psi_{1}\right\|_{H^{3}\left(\mathbb{R}^{5}\right)}^{2}\right) .
\end{aligned}
$$

Invoking Lemma 7.2, we can rewrite (7.4) as

$$
\begin{aligned}
& \frac{d}{d t} \ln \left(1+\left\|u_{1}\right\|_{H^{3}\left(\mathbb{R}^{5}\right)}+\right.\left.\left\|\nabla_{y} \psi_{1}\right\|_{H^{3}\left(\mathbb{R}^{5}\right)}\right) \leq \\
& C\left[1+\left\|\nabla_{x} \times \mathbf{u}\right\|_{\mathrm{BMO}\left(\mathbb{R}^{3}\right)} \ln \left(1+\|\mathbf{u}\|_{H^{3}\left(\mathbb{R}^{3}\right)}\right)\right]
\end{aligned}
$$

Our next step is to prove that

$$
\|\mathbf{u}\|_{H^{3}\left(\mathbb{R}^{3}\right)} \leq C\left[\left\|u_{1}\right\|_{H^{3}\left(\mathbb{R}^{5}\right)}+\left\|\nabla_{y} \psi_{1}\right\|_{H^{3}\left(\mathbb{R}^{5}\right)}\right] .
$$

By (3.12) in Proposition (3.3), it suffices to prove that

$$
\left\|\Delta_{x} \nabla_{x} \mathbf{u}\right\|_{L^{2}\left(\mathbb{R}^{3}\right)} \leq C\left[\left\|u_{1}\right\|_{H^{3}\left(\mathbb{R}^{5}\right)}+\left\|\nabla_{y} \psi_{1}\right\|_{H^{3}\left(\mathbb{R}^{5}\right)}\right] .
$$

In fact, noting that $e_{r}=(\cos \theta, \sin \theta)^{\top}, e_{\theta}=(-\sin \theta, \cos \theta)^{\top}$, one has

$$
\left\{\begin{array}{l}
\partial_{\theta}^{2}\left(e_{r} \otimes e_{r}\right)=-2 e_{r} \otimes e_{r}+2 e_{\theta} \otimes e_{\theta}, \\
\partial_{\theta}^{2}\left(e_{r} \otimes e_{\theta}\right)=-2 e_{r} \otimes e_{\theta}-2 e_{\theta} \otimes e_{r}, \\
\partial_{\theta}^{2}\left(e_{\theta} \otimes e_{r}\right)=-2 e_{r} \otimes e_{\theta}-2 e_{\theta} \otimes e_{r}, \\
\partial_{\theta}^{2}\left(e_{\theta} \otimes e_{\theta}\right)=2 e_{r} \otimes e_{r}-2 e_{\theta} \otimes e_{\theta}, \\
\partial_{\theta}^{2}\left(e_{r} \otimes e_{3}\right)=-e_{r} \otimes e_{3} \\
\partial_{\theta}^{2}\left(e_{\theta} \otimes e_{3}\right)=-e_{\theta} \otimes e_{3} .
\end{array}\right.
$$


It follows from (3.16) that

$$
\begin{aligned}
\Delta_{x} \nabla_{x} \mathbf{u}= & \left(\partial_{r}^{2}+\frac{1}{r} \partial_{r}+\frac{1}{r^{2}} \partial_{\theta}^{2}+\partial_{z}^{2}\right) \nabla_{x} \mathbf{u} \\
= & {\left[\Delta_{x}\left(\partial_{r} u^{r}\right)-\frac{2}{r^{2}} \partial_{r} u^{r}+\frac{2 u^{r}}{r^{3}}\right] e_{r} \otimes e_{r} } \\
& +\left[\Delta_{x}\left(\partial_{r} u^{\theta}\right)-\frac{2}{r^{2}} \partial_{r} u^{\theta}+\frac{2 u^{\theta}}{r^{3}}\right] e_{r} \otimes e_{\theta} \\
& -\left[\Delta_{x}\left(\frac{u^{\theta}}{r}\right)+\frac{2}{r^{2}} \partial_{r} u^{\theta}-\frac{2 u^{\theta}}{r^{3}}\right] e_{\theta} \otimes e_{r} \\
& +\left[\Delta_{x}\left(\frac{u^{r}}{r}\right)+\frac{2}{r^{2}} \partial_{r} u^{r}-\frac{2 u^{r}}{r^{3}}\right] e_{\theta} \otimes e_{\theta} \\
& +\left[\Delta_{x}\left(\partial_{r} u^{z}\right)-\frac{1}{r^{2}} \partial_{r} u^{z}\right] e_{r} \otimes e_{z} \\
& +\left[\Delta_{x}\left(\partial_{z} u^{r}\right)-\frac{1}{r^{2}} \partial_{z} u^{r}\right] e_{z} \otimes e_{r} \\
& +\left[\Delta_{x}\left(\partial_{z} u^{\theta}\right)-\frac{1}{r^{2}} \partial_{z} u^{\theta}\right] e_{z} \otimes e_{\theta}+\Delta_{x}\left(\partial_{z} u^{z}\right) e_{z} \otimes e_{z} .
\end{aligned}
$$

Consequently, we have

$$
\begin{array}{r}
\left\|\Delta_{x} \nabla_{x} \mathbf{u}\right\|_{L^{2}\left(\mathbb{R}^{3}\right)}^{2} \\
=\int_{-\infty}^{\infty} \int_{0}^{\infty}\left|\Delta_{x} \nabla_{x} \mathbf{u}\right|^{2} r d r d z \\
=\left(\int _ { - \infty } ^ { \infty } \int _ { 0 } ^ { \infty } \left(\left|\Delta_{x}\left(\partial_{z} u^{\theta}\right)-\frac{1}{r^{2}} \partial_{z} u^{\theta}\right|^{2}\right.\right. \\
+\left|\Delta_{x}\left(\partial_{r} u^{\theta}\right)-\frac{2}{r^{2}} \partial_{r} u^{\theta}+\frac{2 u^{\theta}}{r^{3}}\right|^{2} \\
\left.\left.+\left|\Delta_{x}\left(\frac{u^{\theta}}{r}\right)+\frac{2}{r^{2}} \partial_{r} u^{\theta}-\frac{2 u^{\theta}}{r^{3}}\right|^{2}\right) r d r d z\right) \\
+\left(\int_{-\infty}^{\infty} \int_{0}^{\infty} \begin{array}{r}
\left(\left|\Delta_{x}\left(\frac{u^{r}}{r}\right)+\frac{2}{r^{2}} \partial_{r} u^{r}-\frac{2 u^{r}}{r^{3}}\right|^{2}\right. \\
+\left|\Delta_{x}\left(\partial_{r} u^{z}\right)-\frac{1}{r^{2}} \partial_{r} u^{z}\right|^{2} \\
+\left|\Delta_{x}\left(\partial_{z} u^{r}\right)-\frac{1}{r^{2}} \partial_{z} u^{r}\right|^{2} \\
+\left|\Delta_{x}\left(\partial_{r} u^{r}\right)-\frac{2}{r^{2}} \partial_{r} u^{r}+\frac{2 u^{r}}{r^{3}}\right|^{2} \\
\left.\left.+\left|\Delta_{x}\left(\partial_{z} u^{z}\right)\right|^{2}\right) r d r d z\right)
\end{array}\right.
\end{array}
$$


First of all, we have

$$
\begin{aligned}
\int_{-\infty}^{\infty} \int_{0}^{\infty}\left|\Delta_{x}\left(\partial_{z} u^{\theta}\right)-\frac{1}{r^{2}} \partial_{z} u^{\theta}\right|^{2} r d r d z & = \\
& \int_{-\infty}^{\infty} \int_{0}^{\infty}\left|\partial_{z} \Delta_{y} u_{1}\right|^{2} r^{3} d r d z
\end{aligned}
$$

On the other hand, direct calculations show that

$$
\begin{aligned}
& \int_{-\infty}^{\infty} \int_{0}^{\infty}\left(\left|\Delta_{x}\left(\partial_{r} u^{\theta}\right)-\frac{2}{r^{2}} \partial_{r} u^{\theta}+\frac{2 u^{\theta}}{r^{3}}\right|^{2}\right. \\
& \left.\quad+\left|\Delta_{x}\left(\frac{u^{\theta}}{r}\right)+\frac{2}{r^{2}} \partial_{r} u^{\theta}-\frac{2 u^{\theta}}{r^{3}}\right|^{2}\right) r d r d z \\
& =\int_{-\infty}^{\infty} \int_{0}^{\infty}\left(\left|r \partial_{r} \Delta_{y} u_{1}+\Delta_{y} u_{1}\right|^{2}+\left|\Delta_{y} u_{1}\right|^{2}\right) r d r d z \\
& \leq 2 \int_{-\infty}^{\infty} \int_{0}^{\infty}\left|\partial_{r} \Delta_{y} u_{1}\right|^{2} r^{3} d r d z+3 \int_{-\infty}^{\infty} \int_{0}^{\infty}\left|\frac{\Delta_{y} u_{1}}{r}\right|^{2} r^{3} d r d z \\
& \leq C \int_{-\infty}^{\infty} \int_{0}^{\infty}\left|\partial_{r} \Delta_{y} u_{1}\right|^{2} r^{3} d r d z
\end{aligned}
$$

where we have used the following Hardy's inequality:

$$
\left\|\frac{w}{r}\right\|_{L^{2}\left(\mathbb{R}^{5}\right)} \leq\left\|w_{r}\right\|_{L^{2}\left(\mathbb{R}^{5}\right)}, \quad w \in H^{1}\left(\mathbb{R}^{5}\right),
$$

which can be verified directly by performing integration by parts in the $r$-direction. Consequently, we have

$$
\mathrm{I} \leq C\left\|\nabla_{y} \Delta_{y} u_{1}\right\|_{L^{2}\left(\mathbb{R}^{5}\right)}^{2}
$$

Similarly, as in (7.10), one has

$$
\begin{aligned}
\int_{-\infty}^{\infty} \int_{0}^{\infty}\left|\Delta_{x}\left(\partial_{z} u^{r}\right)-\frac{1}{r^{2}} \partial_{z} u^{r}\right|^{2} r d r d z & = \\
& \int_{-\infty}^{\infty} \int_{0}^{\infty}\left|\partial_{z} \Delta_{y} \partial_{z} \psi_{1}\right|^{2} r^{3} d r d z
\end{aligned}
$$

Moreover, it is easy to see that

$$
\begin{aligned}
\left|\Delta_{x}\left(\partial_{r} u^{z}\right)-\frac{1}{r^{2}} \partial_{r} u^{z}\right|^{2} & =r^{2}\left|\left(\partial_{r}^{2}+\frac{3}{r} \partial_{r}\right) \Delta_{y} \psi_{1}\right|^{2} \\
& \leq r^{2}\left|\Delta_{y}^{2} \psi_{1}\right|^{2}+r^{2}\left|\partial_{z}^{2} \Delta_{y} \psi_{1}\right|^{2} .
\end{aligned}
$$


Finally, we compute

$$
\begin{aligned}
\left|\Delta_{x}\left(\frac{u^{r}}{r}\right)+\frac{2}{r^{2}} \partial_{r} u^{r}-\frac{2 u^{r}}{r^{3}}\right|^{2} \\
+\left|\Delta_{x}\left(\partial_{r} u^{r}\right)-\frac{2}{r^{2}} \partial_{r} u^{r}+\frac{2 u^{r}}{r^{3}}\right|^{2}+\left|\Delta_{x}\left(\partial_{z} u^{z}\right)\right|^{2} \\
=\left|\Delta_{x}\left(\frac{u^{r}}{r}\right)+\frac{2}{r^{2}} \partial_{r} u^{r}-\frac{2 u^{r}}{r^{3}}\right|^{2} \\
+\left|\Delta_{x}\left(\partial_{r} u^{r}\right)-\frac{2}{r^{2}} \partial_{r} u^{r}+\frac{2 u^{r}}{r^{3}}\right|^{2} \\
+\mid\left[\Delta_{x}\left(\partial_{r} u^{r}\right)-\frac{2}{r^{2}} \partial_{r} u^{r}+\frac{2 u^{r}}{r^{3}}\right] \\
\quad+\left.\left[\Delta_{x}\left(\frac{u^{r}}{r}\right)+\frac{2}{r^{2}} \partial_{r} u^{r}-\frac{2 u^{r}}{r^{3}}\right]\right|^{2} \\
\leq 3\left|\Delta_{x}\left(\frac{u^{r}}{r}\right)+\frac{2}{r^{2}} \partial_{r} u^{r}-\frac{2 u^{r}}{r^{3}}\right|^{2} \\
+\quad 3\left|\Delta_{x}\left(\partial_{r} u^{r}\right)-\frac{2}{r^{2}} \partial_{r} u^{r}+\frac{2 u^{r}}{r^{3}}\right|^{2} \\
=3\left|\Delta_{y} \partial_{z} \psi_{1}\right|^{2}+3\left|r \partial_{r} \Delta_{y} \partial_{z} \psi_{1}+\Delta_{y} \partial_{z} \psi_{1}\right|^{2} \\
\leq 9\left|\Delta_{y} \partial_{z} \psi_{1}\right|^{2}+6 r^{2}\left|\partial_{r} \Delta_{y} \partial_{z} \psi_{1}\right|^{2} .
\end{aligned}
$$

Thus we have

$$
\mathrm{II} \leq C\left\|\nabla_{y} \psi_{1}\right\|_{H^{3}\left(\mathbb{R}^{5}\right)}^{2} .
$$

Combining (7.14) with (7.18), we prove (7.7). The theorem now follows directly from (7.6) and (7.7).

\section{A Nonblowup Criterion of Prodi-Serrin Type}

There are many results on the global regularity of the solutions of the threedimensional Navier-Stokes equations under some additional conditions imposed on the solution. In particular, the papers of Prodi [29] and Serrin [31] give the following Prodi-Serrin's nonblowup criterion for the solution of the three-dimensional Navier-Stokes equations:

Any Leray-Hopf solution $u$ to the three-dimensional Navier-Stokes equations (2.1) on $[0, T]$ is smooth on $[0, T]$ if $\|\mathbf{u}\|_{L_{t}^{q} L_{x}^{p}\left([0, T] \times \mathbb{R}^{3}\right)}$ $<\infty$ for some $p, q$ satisfying $\frac{3}{p}+\frac{2}{q} \leq 1,3<p \leq \infty$. 
A local version was later established by Serrin [30] for $\frac{3}{p}+\frac{2}{q}<1$ and by Struwe [32] for $\frac{3}{p}+\frac{2}{q}=1$. The highly nontrivial endpoint case of $p=3$ was recently established by Iskauriaza, Serëgin, and Shverak [13].

To demonstrate the similarity between the three-dimensional model equations (3.1) and the axisymmetric Navier-Stokes equations (2.3)-(2.4), we will prove a nonblowup criterion of the Prodi-Serrin type for our model. Here we focus only on the whole space case with $3<p \leq \infty$.

THEOREM 8.1 Assume that $\left(u_{1}, \omega_{1}, \psi_{1}\right)$ is a weak solution to the three-dimensional model equations (3.1) on $[0, T]$. Then $\left(u_{1}, \omega_{1}, \psi_{1}\right)$ is smooth on $[0, T] \times \mathbb{R}^{3}$ if $u^{\theta}$ satisfies the condition

$$
\left\|u^{\theta}\right\|_{L_{t}^{q} L_{x}^{p}\left([0, T] \times \mathbb{R}^{3}\right)}<\infty
$$

for some $p, q$ satisfying $\frac{3}{p}+\frac{2}{q} \leq 1,3<p \leq \infty$.

PROOF: In the case of $p=\infty$, by Hardy's inequality (7.13) and the CalderonZygmund theorem, we have

$$
\begin{aligned}
& \frac{1}{2} \frac{d}{d t} \int_{\mathbb{R}^{5}}\left(\left|\nabla_{y} u_{1}\right|^{2}+\left|\omega_{1}\right|^{2}\right) d y+\int_{\mathbb{R}^{5}}\left(\left|\Delta_{y} u_{1}\right|^{2}+\left|\nabla_{y} \omega_{1}\right|^{2}\right) d y \\
&=-\int_{\mathbb{R}^{5}}\left(2 \partial_{z} \psi_{1} u_{1} \Delta_{y} u_{1}+u_{1}^{2} \partial_{z} \omega_{1}\right) d y \\
& \leq\left\|u^{\theta}\right\|_{L^{\infty}}\left(\left\|\Delta_{y} u_{1}\right\|_{L^{2}\left(\mathbb{R}^{5}\right)}+\left\|\nabla_{y} \omega_{1}\right\|_{L^{2}\left(\mathbb{R}^{5}\right)}\right) \\
& \quad \times\left(\left\|\frac{\partial_{z} \psi_{1}}{r}\right\|_{L^{2}\left(\mathbb{R}^{5}\right)}+\left\|\frac{u_{1}}{r}\right\|_{L^{2}\left(\mathbb{R}^{5}\right)}\right) \\
& \leq C\left\|u^{\theta}\right\|_{L^{\infty}}\left(\left\|\Delta_{y} u_{1}\right\|_{L^{2}\left(\mathbb{R}^{5}\right)}+\left\|\nabla_{y} \omega_{1}\right\|_{L^{2}\left(\mathbb{R}^{5}\right)}\right) \\
& \quad \times\left(\left\|\Delta_{y} \psi_{1}\right\|_{L^{2}\left(\mathbb{R}^{5}\right)}+\left\|\nabla_{y} u_{1}\right\|_{L^{2}\left(\mathbb{R}^{5}\right)}\right) \\
& \leq C\left\|u^{\theta}\right\|_{L^{\infty}}^{2}\left(\left\|\omega_{1}\right\|_{L^{2}\left(\mathbb{R}^{5}\right)}+\left\|\nabla_{y} u_{1}\right\|_{L^{2}\left(\mathbb{R}^{5}\right)}\right)^{2} \\
& \quad+\frac{1}{2}\left(\left\|\Delta_{y} u_{1}\right\|_{L^{2}\left(\mathbb{R}^{5}\right)}+\left\|\nabla_{y} \omega_{1}\right\|_{L^{2}\left(\mathbb{R}^{5}\right)}\right)^{2}
\end{aligned}
$$

which gives

$$
\begin{aligned}
& \left\|\omega_{1}(T)\right\|_{L^{2}\left(\mathbb{R}^{5}\right)}+\left\|\nabla_{y} u_{1}(T)\right\|_{L^{2}\left(\mathbb{R}^{5}\right)} \leq \\
& \quad C\left(\left\|\Delta_{y} \psi_{10}\right\|_{L^{2}\left(\mathbb{R}^{5}\right)}+\left\|\nabla_{y} u_{10}\right\|_{L^{2}\left(\mathbb{R}^{5}\right)}\right) \exp \left\{C \int_{0}^{T}\left\|u^{\theta}(s)\right\|_{L^{\infty}}^{2} d s\right\} .
\end{aligned}
$$


Similarly, in the case of $3<p<\infty$, we have

$$
\begin{aligned}
& \frac{1}{2} \frac{d}{d t} \int_{\mathbb{R}^{5}}\left(\left|\nabla_{y} u_{1}\right|^{2}+\left|\omega_{1}\right|^{2}\right) d y+\int_{\mathbb{R}^{5}}\left(\left|\Delta_{y} u_{1}\right|^{2}+\left|\nabla_{y} \omega_{1}\right|^{2}\right) d y \\
& \leq\left(\left\|\Delta_{y} u_{1}\right\|_{L^{2}\left(\mathbb{R}^{5}\right)}+\left\|\nabla_{y} \omega_{1}\right\|_{L^{2}\left(\mathbb{R}^{5}\right)}\right) \\
& \times\left\|\frac{u^{\theta}}{r^{2 / p}}\right\|_{L^{p}\left(\mathbb{R}^{5}\right)}\left(\left\|\frac{\partial_{z} \psi_{1}}{r^{1-2 / p}}\right\|_{L^{\frac{2 p}{p-2}\left(\mathbb{R}^{5}\right)}}+\left\|\frac{u_{1}}{r^{1-\frac{2}{p}}}\right\|_{L^{\frac{2 p}{p-2}\left(\mathbb{R}^{5}\right)}}\right) \\
& \leq C\left\|u^{\theta}\right\|_{L^{p}\left(\mathbb{R}^{3}\right)}\left(\left\|\partial_{z} \psi_{1}\right\|_{L^{\frac{2 p}{p-2}}\left(\mathbb{R}^{3}\right)}+\left\|u_{1}\right\|_{L^{\frac{2 p}{p-2}}\left(\mathbb{R}^{3}\right)}\right) \\
& \times\left(\left\|\Delta_{y} u_{1}\right\|_{L^{2}\left(\mathbb{R}^{5}\right)}+\left\|\nabla_{y} \omega_{1}\right\|_{L^{2}\left(\mathbb{R}^{5}\right)}\right) .
\end{aligned}
$$

Further, we note that

$$
\begin{aligned}
\int_{-\infty}^{\infty} & \int_{0}^{\infty}\left|u_{1}\right|^{\frac{2 p}{p-2}} r d r d z \\
\leq & \frac{p}{p-2} \int_{-\infty}^{\infty} \int_{0}^{\infty}\left|u_{1}\right|^{\frac{p+2}{p-2}}\left|\partial_{r} u_{1}\right| r^{2} d r d z \\
\leq & \frac{p}{p-2}\left(\int_{-\infty}^{\infty} \int_{0}^{\infty}\left|u_{1}\right|^{\frac{2 p}{p-2}} r d r d z\right)^{\frac{p+2}{2 p}} \\
& \cdot\left(\int_{-\infty}^{\infty} \int_{0}^{\infty}\left|r \partial_{r} u_{1}\right|^{\frac{2 p}{p-2}} r d r d z\right)^{\frac{p-2}{2 p}}
\end{aligned}
$$

which gives

$$
\int_{-\infty}^{\infty} \int_{0}^{\infty}\left|u_{1}\right|^{\frac{2 p}{p-2}} r d r d z \leq C \int_{-\infty}^{\infty} \int_{0}^{\infty}\left|r \partial_{r} u_{1}\right|^{\frac{2 p}{p-2}} r d r d z
$$

Observe that $2<\frac{2 p}{p-2}<6$ if $3<p<\infty$. Thus, by the standard interpolation inequality and Hardy's inequality (7.13), we have

$$
\begin{aligned}
& \left(\int_{-\infty}^{\infty} \int_{0}^{\infty}\left|r \partial_{r} u_{1}\right|^{\frac{2 p}{p-2}} r d r d z\right)^{\frac{p-2}{2 p}} \\
& \quad \leq C\left\|r \partial_{r} u_{1}\right\|_{L^{2}\left(\mathbb{R}^{3}\right)}^{1-3 / p}\left\|\nabla\left(r \partial_{r} u_{1}\right)\right\|_{L^{2}\left(\mathbb{R}^{3}\right)}^{3 / p} \\
& \quad \leq C\left\|\nabla_{y} u_{1}\right\|_{L^{2}\left(\mathbb{R}^{5}\right)}^{1-3 / p}\left(\left\|\Delta_{y} u_{1}\right\|_{L^{2}\left(\mathbb{R}^{5}\right)}^{3 / p}+\left\|\frac{\partial_{r} u_{1}}{r}\right\|_{L^{2}\left(\mathbb{R}^{5}\right)}^{3 / p}\right) \\
& \quad \leq C\left\|\nabla_{y} u_{1}\right\|_{L^{2}\left(\mathbb{R}^{5}\right)}^{1-3 / p}\left\|\Delta_{y} u_{1}\right\|_{L^{2}\left(\mathbb{R}^{5}\right)}^{3 / p} .
\end{aligned}
$$


Therefore, we obtain

$$
\begin{aligned}
& \frac{1}{2} \frac{d}{d t} \int_{\mathbb{R}^{5}}\left(\left|\nabla_{y} u_{1}\right|^{2}+\left|\omega_{1}\right|^{2}\right) d y+\int_{\mathbb{R}^{5}}\left(\left|\Delta_{y} u_{1}\right|^{2}+\left|\nabla_{y} \omega_{1}\right|^{2}\right) d y \\
& \leq C\left\|u^{\theta}\right\|_{L^{p}\left(\mathbb{R}^{3}\right)}\left(\left\|\partial_{z} \nabla_{y} \psi_{1}\right\|_{L^{2}\left(\mathbb{R}^{3}\right)}+\left\|\nabla_{y} u_{1}\right\|_{L^{2}\left(\mathbb{R}^{3}\right)}\right)^{\frac{p-3}{p}} \\
& \quad \times\left(\left\|\Delta_{y} u_{1}\right\|_{L^{2}\left(\mathbb{R}^{5}\right)}+\left\|\nabla_{y} \omega_{1}\right\|_{L^{2}\left(\mathbb{R}^{5}\right)}\right)^{1+\frac{3}{p}} \\
& \leq C\left\|u^{\theta}\right\|_{L^{p}\left(\mathbb{R}^{3}\right)}^{\frac{2 p}{p-3}}\left(\left\|\partial_{z} \nabla_{y} \psi_{1}\right\|_{L^{2}\left(\mathbb{R}^{3}\right)}+\left\|\nabla_{y} u_{1}\right\|_{L^{2}\left(\mathbb{R}^{3}\right)}\right)^{2} \\
& \quad+\frac{1}{2}\left(\left\|\Delta_{y} u_{1}\right\|_{L^{2}\left(\mathbb{R}^{5}\right)}+\left\|\nabla_{y} \omega_{1}\right\|_{L^{2}\left(\mathbb{R}^{5}\right)}\right)^{2} .
\end{aligned}
$$

The theorem now follows from the Gronwall inequality and the assumption of the theorem.

Acknowledgment. This work was done when Zhen Lei was visiting Applied \& Computational Mathematics at the California Institute of Technology. He would like to thank the institute for its hospitality. The authors were in part supported by the National Science Foundation under grants FRG DMS-0353838, ITR ACI0204932, and DMS-0713670. We would like to thank Prof. Hector Ceniceros, Congming $\mathrm{Li}$, and the two anonymous referees for their valuable comments and suggestions on the original manuscript.

\section{Bibliography}

[1] Beale, J. T.; Kato, T.; Majda, A. Remarks on the breakdown of smooth solutions for the 3-D Euler equations. Comm. Math. Phys. 94 (1984), no. 1, 61-66.

[2] Caffarelli, L.; Kohn, R.; Nirenberg, L. Partial regularity of suitable weak solutions of the NavierStokes equations. Comm. Pure Appl. Math. 35 (1982), no. 6, 771-831.

[3] Cao, C.; Titi, E. S. Global well-posedness of the three-dimensional primitive equations of large scale ocean and atmosphere dynamics. Ann. of Math. (2) 166 (2007), no. 1, 245-267.

[4] Chen, C.-C.; Strain, R. M.; Tsai, T.; Yau, H.-T. Lower bounds on the blow-up rate of the axisymmetric Navier-Stokes equations II. arXiv: math.AP/0709.4230v1, 2007.

[5] Chen, C.-C.; Strain, R. M.; Yau, H.-T.; Tsai, T.-P. Lower bound on the blow-up rate of the axisymmetric Navier-Stokes equations. Int. Math. Res. Notes 2008. Available online at: http://imrn.oxfordjournals.org/cgi/content/refs/2008/ rnn016/rnn016. arXiv: math.AP/0701796v1, 2007.

[6] Constantin, P. Note on loss of regularity for solutions of the 3-D incompressible Euler and related equations. Comm. Math. Phys. 104 (1986), no. 2, 311-326.

[7] Constantin, P.; Foias, C. Navier-Stokes equations. Chicago Lectures in Mathematics. Chicago University, Chicago, 1988.

[8] De Gregorio, S. On a one-dimensional model for the three-dimensional vorticity equation. J. Statist. Phys. 59 (1990), no. 5-6, 1251-1263.

[9] De Gregorio, S. A partial differential equation arising in a $1 \mathrm{D}$ model for the $3 \mathrm{D}$ vorticity equation. Math. Methods Appl. Sci. 19 (1996), no. 15, 1233-1255.

[10] Deng, J.; Hou, T. Y.; Yu, X. Geometric properties and non-blowup of 3D incompressible Euler flow. Comm. Partial Differential Equations 30 (2005), no. 1-3, 225-243. 
[11] Deng, J.; Hou, T. Y.; Yu, X. Improved geometric conditions for non-blowup of the 3D incompressible Euler equation. Comm. Partial Differential Equations 31 (2006), no. 1-3, 293-306.

[12] Fefferman, C. Existence and smoothness of the Navier-Stokes equation. The millennium prize problems, 57-67. Clay Mathematics Institute, Cambridge, Mass., 2006. Available online at: http://www.claymath.org/millennium/Navier-Stokes_ Equations/navierstokes.pdf

[13] Iskauriaza, L.; Serëgin, G. A.; Shverak, V. $L_{3, \infty}$-solutions of Navier-Stokes equations and backward uniqueness. Uspekhi Mat. Nauk 58 (2003), no. 2(350), 3-44; translation in Russian Math. Surveys $\mathbf{5 8}$ (2003), no. 2, 211-250.

[14] Hou, T. Y.; Li, R. Dynamic depletion of vortex stretching and non-blowup of the 3-D incompressible Euler equations. J. Nonlinear Sci. 16 (2006), no. 6, 639-664.

[15] Hou, T. Y.; Li, R. Computing nearly singular solutions using pseudo-spectral methods. J. Comput. Phys. 226 (2007), no. 1, 379-397.

[16] Hou, T. Y.; Lei, Z. On the partial regularity of a 3D model of the Navier-Stokes equations. Comm. Math Phys., submitted. Available online at: http://www.acm.caltech.edu/ $\sim$ hou/papers/ModelNS-PR-Final.pdf

[17] Hou, T. Y.; Lei, Z.; Li, C.-M. Global regularity of the 3D axi-symmetric NavierStokes equations with anisotropic initial data. Comm. Partial Differential Equations, forthcoming. Available online at: http://www.acm.caltech.edu/ hou/ papers/NSE_anisotropic_revise1.pdf

[18] Hou, T. Y.; Li, C.-M. Dynamic stability of the 3D axi-symmetric Navier-Stokes equations with swirl. Comm. Pure Appl. Math. 61 (2008), no. 5, 661-697.

[19] Hou, T. Y.; Wetton, B. T. R. Convergence of a finite difference scheme for the Navier-Stokes equations using vorticity boundary conditions. SIAM J. Numer. Anal. 29 (1992), no. 3, 615-639.

[20] Kerr, R. M. Evidence for a singularity of the three-dimensional, incompressible Euler equations. Phys. Fluids A 5 (1993), no. 7, 1725-1746.

[21] Koch, G.; Nadirashvili, N.; Seregin, G.; Sverak, V. Liouville theorems for the Navier-Stokes equations and applications. arXiv: math.AP/0709.3599v1, 2007.

[22] Kozono, H.; Taniuchi, Y. Bilinear estimates in BMO and the Navier-Stokes equations. Math. Z. 235 (2000), no. 1, 173-194.

[23] Ladyžhenskaja, O. A. Mathematical questions of the dynamics of a viscous incompressible fluid. 2nd revised and supplemented ed. Nauka, Moscow, 1970.

[24] Lin, F. A new proof of the Caffarelli-Kohn-Nirenberg theorem. Comm. Pure Appl. Math. 51 (1998), no. 3, 241-257.

[25] Liu, J.-G.; Wang, W.-C. Convergence analysis of the energy and helicity preserving scheme for axisymmetric flows. SIAM J. Numer. Anal. 44 (2006), no. 6, 2456-2480 (electronic).

[26] Majda, A. J.; Bertozzi, A. L. Vorticity and incompressible flow. Cambridge Texts in Applied Mathematics, 27. Cambridge University, Cambridge, Mass., 2002.

[27] Okamoto, H.; Ohkitani, K. On the role of the convection term in the equations of motion of incompressible fluid. J. Phys. Soc. Japan 74 (2005), no. 10, 2737-2742.

[28] Orszag, S. A.; Israeli, M. Numerical simulation of viscous incompressible flows. Ann. Rev. Fluid Mech. 6 (1974), 281-318. Available online at: http://arjournals. annualreviews.org/doi/pdf/10.1146/annurev.fl.06.010174.001433? cookieset $=1$

[29] Prodi, G. Un teorema di unicità per le equazioni di Navier-Stokes. Ann. Mat. Pura Appl. (4) 48 (1959), 173-182.

[30] Serrin, J. On the interior regularity of weak solutions of the Navier-Stokes equations. Arch. Rational Mech. Anal. 9 (1962), 187-195. 
[31] Serrin, J. The initial value problem for the Navier-Stokes equations. Nonlinear Problems (Proc. Sympos., Madison, Wis.), 69-98. University of Wisconsin, Madison, Wis., 1963.

[32] Struwe, M. On partial regularity results for the Navier-Stokes equations. Comm. Pure Appl. Math. 41 (1988), no. 4, 437-458.

[33] Temam, R. Navier-Stokes equations. Theory and numerical analysis. Reprint of the 1984 ed. AMS Chelsea, Providence, R.I., 2001.

[34] van Dommelen, L. L.; Shen, S. F. The spontaneous generation of the singularity in a separating laminar boundary layer. J. Comput. Phys. 38 (1980), no. 2, 125-140.

\section{Thomas Y. Hou}

California Institute of Technology

Applied and Computational

Mathematics

1200 E. California Boulevard

MC 217-50

Pasadena, CA 91125

E-mail: hou@acm.caltech.edu

\section{ZHEN LEI}

Northeast Normal University

School of Mathematics and Statistics

Changchun 130024

P.R. CHINA

and

School of Mathematical Sciences

Fudan University

Shanghai 200433

P.R. CHINA

E-mail: leizhn@yahoo.com

Received November 2007.

Revised March 2008. 